Supporting Information for:

\title{
[1,5]-sigmatropic Shifts Regulated by Built-in Frustration
}

\author{
Dinesh V. Vidhani, ${ }^{\star *}$ Jared R. Gillett, ${ }^{\ddagger}$ Yanet Cusido, ${ }^{\dagger}$ Igor V. Alabugin ${ }^{\dagger{ }^{*}}$ \\ †Department of Math \& Natural Science, Miami Dade College, Miami, Florida; \\ †† Department of Chemistry \& Biochemistry, Florida State University, Tallahassee, Florida. \\ ${ }^{\ddagger}$ Department of Chemistry \& Biochemistry, University of Wisconsin, La Crosse, Wisconsin.
}

All geometries were optimized at the B3LYP/LANL2DZ levels which performs well for the estimation of reaction and activation energies of organic reactions ${ }^{1}$ (e.g. Tantillo, J. Am. Chem. Soc., 2006, 128, 6172; Gutta, P. and Tantillo, D. J. Angew. Chem. Int. Ed., 2005, 44, 2719; Leverett, C. A.; Purohit, V. C.; Johnson, A. J.; Davis, R. L.; Tantillo, D. J.; Romo, D. J. Am. Chem. Soc., 2012, 134, 13348) using Gaussian 03 program (see reference). NBO analysis was used to determine electronic structures and properties. ${ }^{2}$ Force Field calculation indicated that optimized structures were found to be true minima with no imaginary frequency. All energies have been expressed in hartrees and the frequencies in $\mathrm{cm}^{-1}$.

Gaussian 09, Gaussian 09, Revision E.01,M. J. Frisch, G. W. Trucks, H. B. Schlegel, G. E. Scuseria, M. A. Robb, J. R. Cheeseman, G. Scalmani, V. Barone, B. Mennucci, G. A. Petersson, H. Nakatsuji, M. Caricato, X. Li, H. P. Hratchian, A. F. Izmaylov, J. Bloino, G. Zheng, J. L. Sonnenberg, M. Hada, M. Ehara, K. Toyota, R. Fukuda, J. Hasegawa, M. Ishida, T. Nakajima, Y. Honda, O. Kitao, H. Nakai, T. Vreven, J. A. Montgomery, Jr., J. E. Peralta, F. Ogliaro, M. Bearpark, J. J. Heyd, E. Brothers, K. N. Kudin, V. N. Staroverov, T. Keith, R. Kobayashi, J. Normand, K. Raghavachari, A. Rendell, J. C. Burant, S. S. Iyengar, J. Tomasi, M. Cossi, N. Rega, J. M. Millam, M. Klene, J. E. Knox, J. B. Cross, V. Bakken, C. Adamo, J. Jaramillo, R. Gomperts, R. E. Stratmann, O. Yazyev, A. J. Austin, R. Cammi, C. Pomelli, J. W. Ochterski, R. L. Martin, K. Morokuma, V. G. Zakrzewski, G. A. Voth, P. Salvador, J. J. Dannenberg, S. Dapprich, A. D. Daniels, O. Farkas, J. B. Foresman, J. V. Ortiz, J. Cioslowski, and D. J. Fox, Gaussian, Inc., Wallingford CT, 2013. 


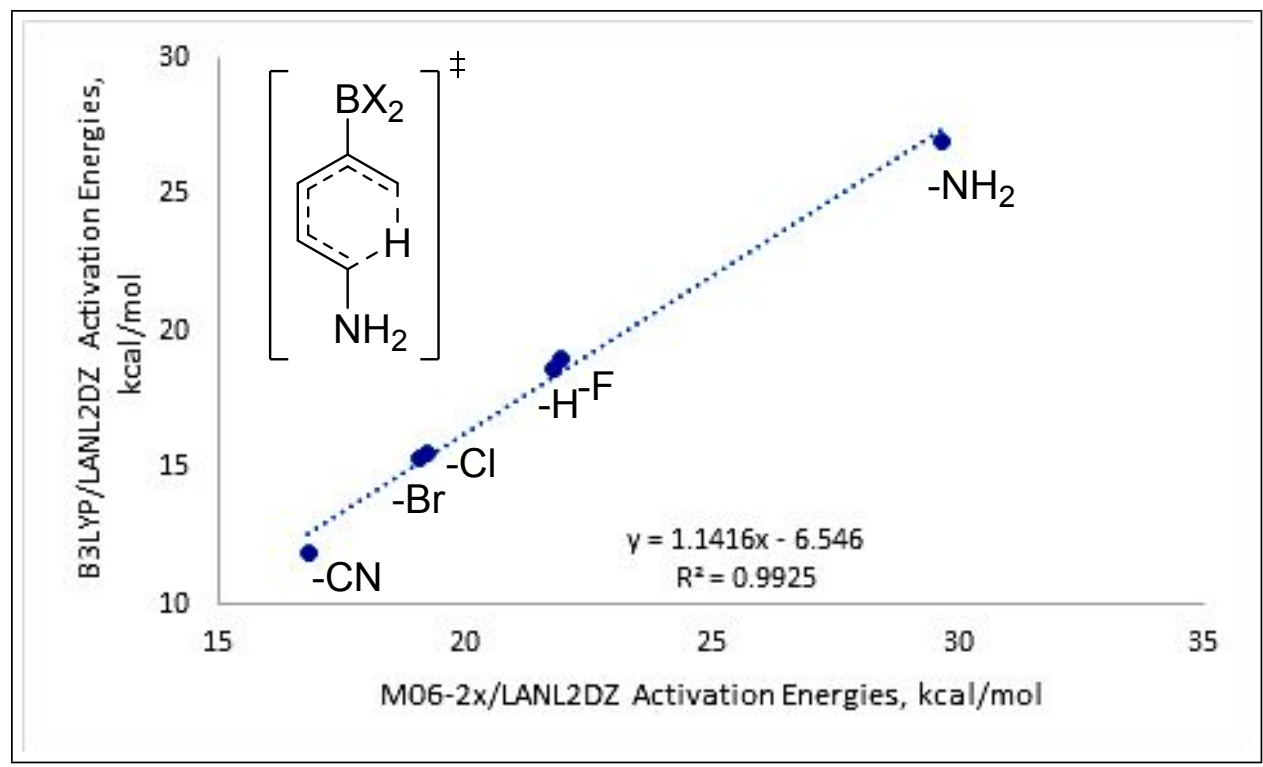

Figure S1. Correlation between M06-2X/LANL2DZ and B3LYP/LANL2DZ calculated activation energies.

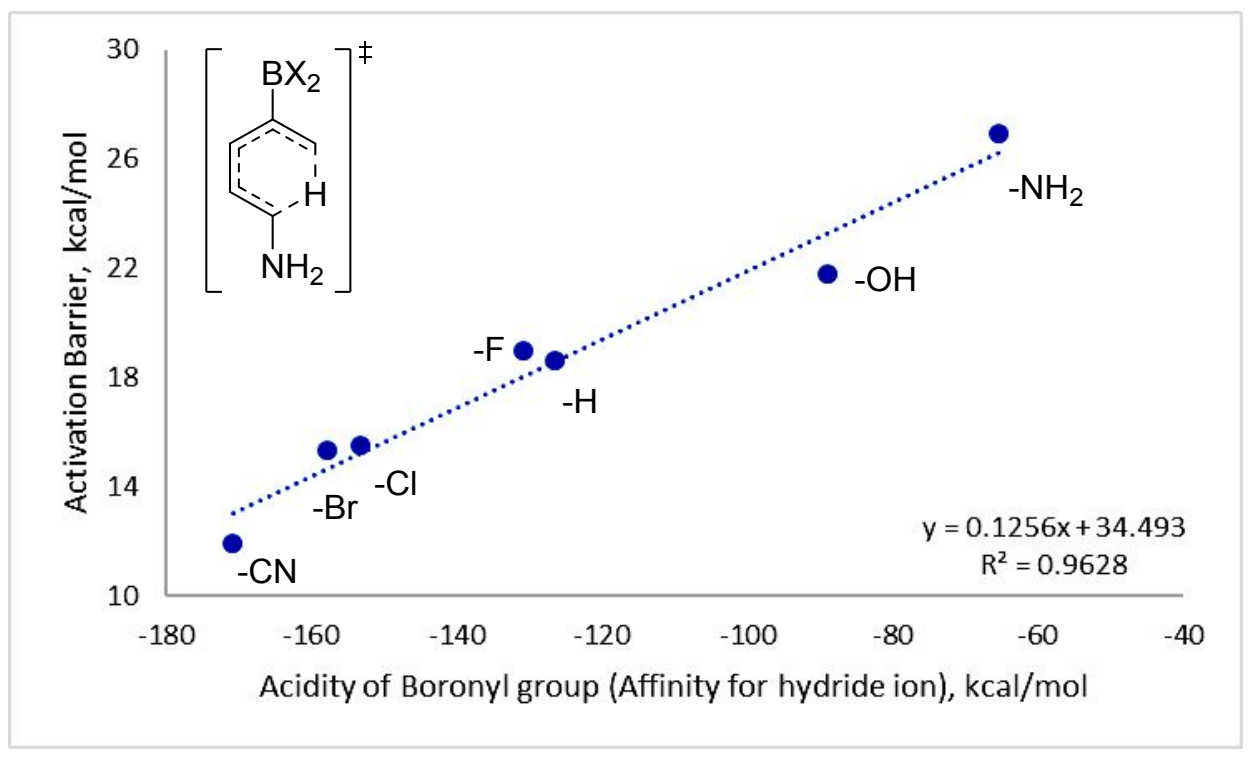

Figure S2. Correlation between activation energies of [1,5] hydride shift and the acidity of boron of $\mathrm{C} 1 / \mathrm{C} 4$ substituted 1,3 pentadienes. 


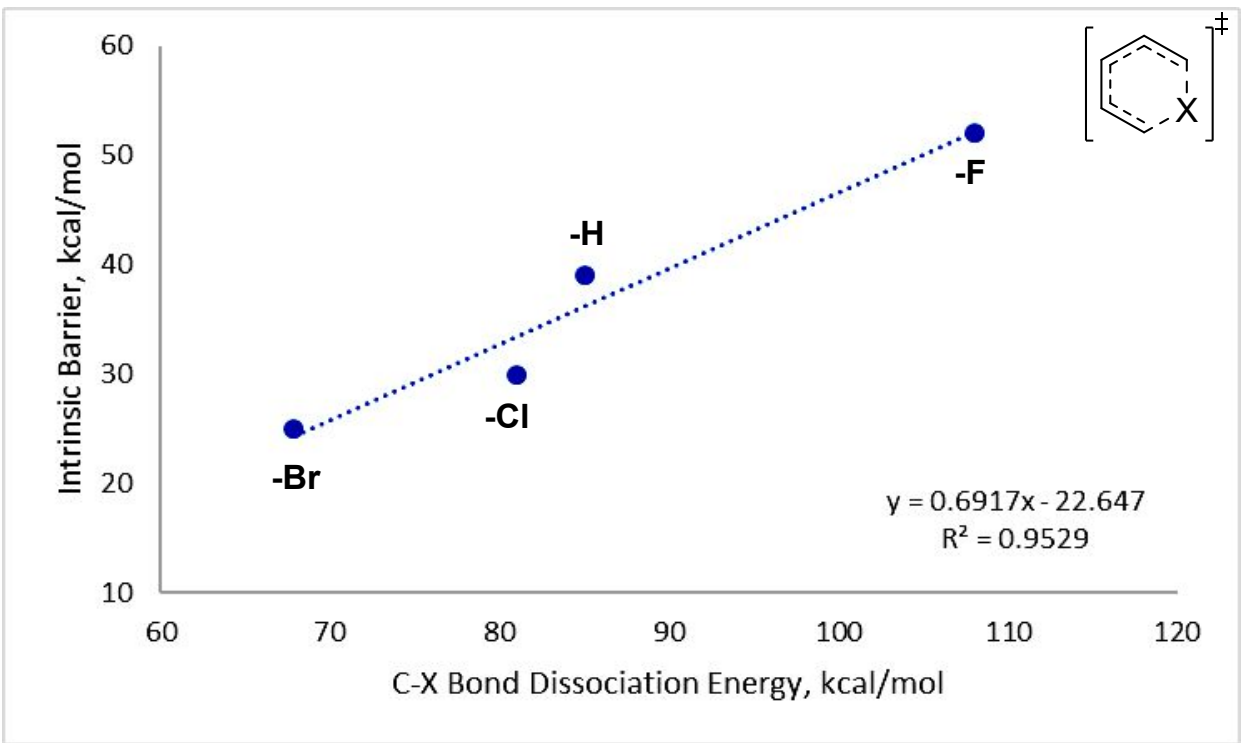

Figure S3. Correlation between $\mathrm{C}-\mathrm{X}$ bond dissociation energies and activation energies of degenerate $[1,5]$ Sigmatropic shifts involving migration of $-\mathrm{Br},-\mathrm{Cl},-\mathrm{H}$ and $-\mathrm{F}$.

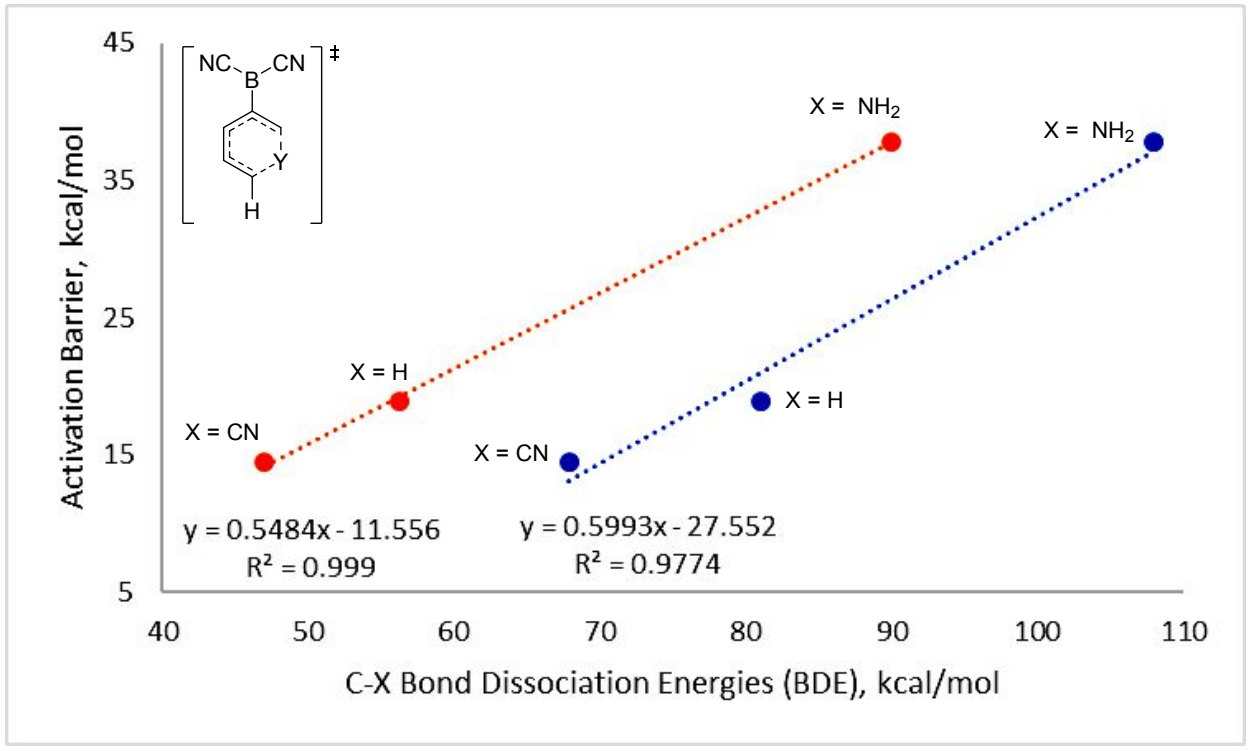

Figure S4. Correlation demonstrating effect of most acidic boronyl group $\left[-\mathrm{B}(\mathrm{CN})_{2}\right]$ on the migratory aptitude of halogens. The BDE calculated for dissociation of $\mathrm{C}-\mathrm{X}$ bond in propenyl halide is shown by orange plot whereas the blue plot corresponds to literature values of $C-X$ bond dissociation energies in typical methyl halide. 


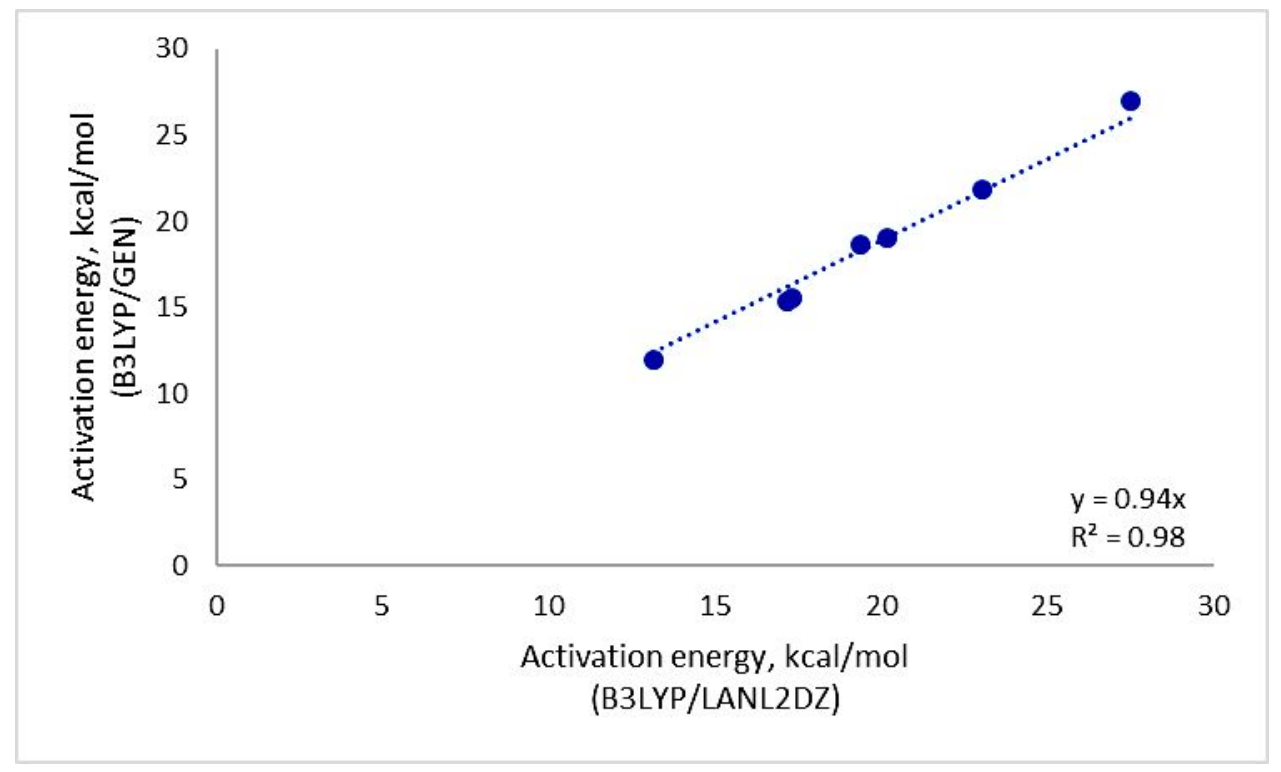

Figure S5. Correlation between B3LYP calculated activation energies using LANL2DZ and split basis set. x-axis: B3LYP/LANL2DZ, y-axis: B3LYP/GEN [B, C, H, N, O and F: 6-311++G(d,p) and Br: LANL2DZ]. Energies are expressed in $\mathrm{kcal} / \mathrm{mol}$.
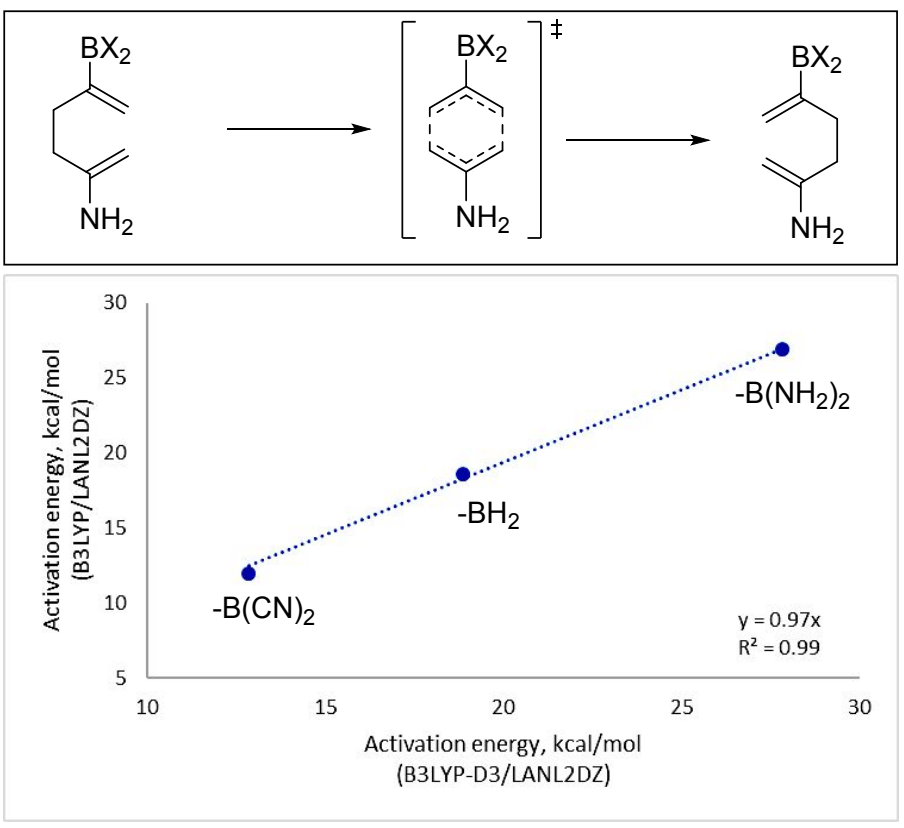

Figure S6. Correlation between B3LYP-D3/LANL2DZ and B3LYP/LANL2DZ calculated energies for selected substrates. Energies are expressed in $\mathrm{kcal} / \mathrm{mol}$ 
Table S1 Cartesian coordinates, total energies, enthalpies, ZPE corrected energies and free energies calculated at the B3LYP/LANL2DZ level for the substrates consisting of -BX2 substituent at $\mathrm{C} 4$ carbon.

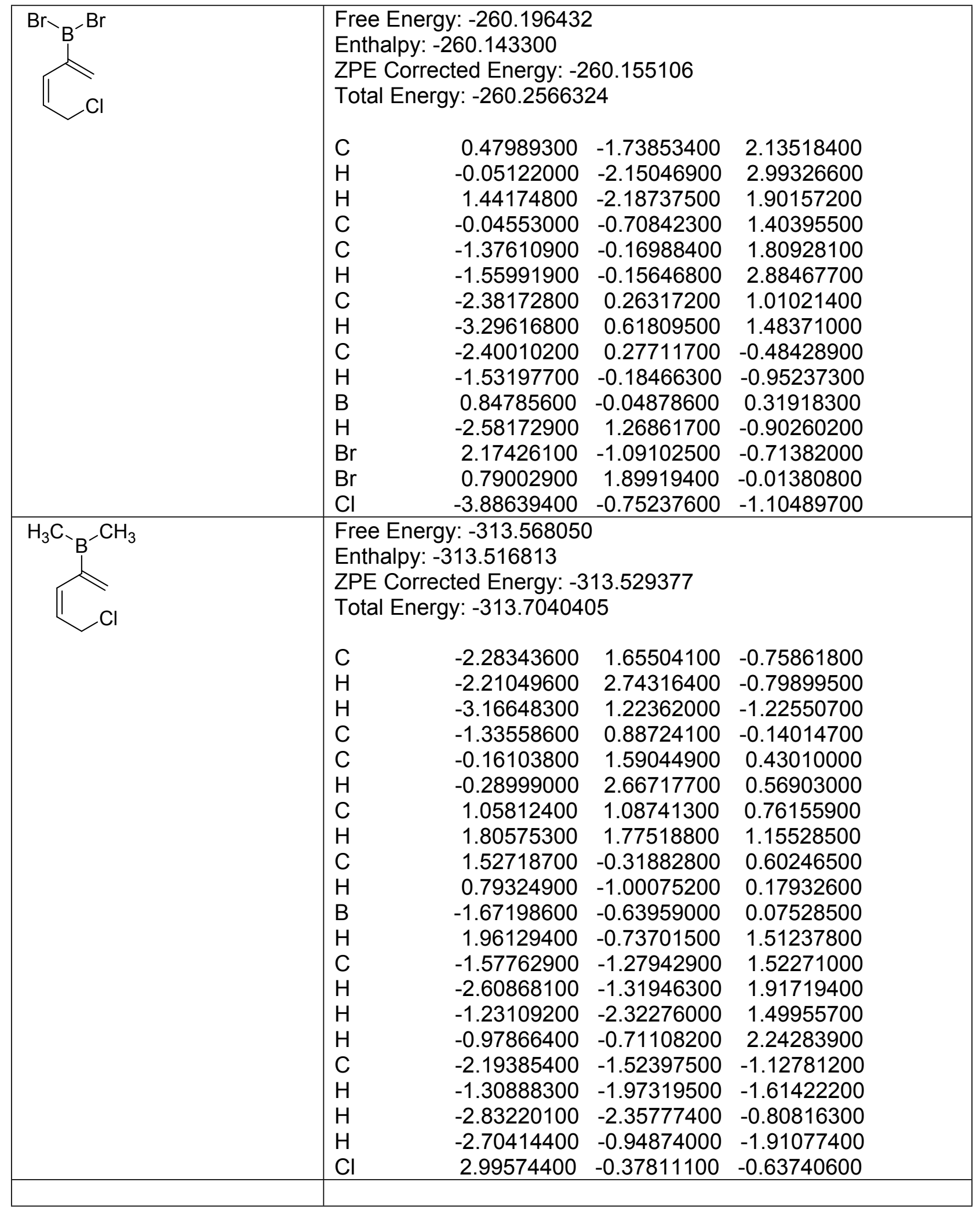




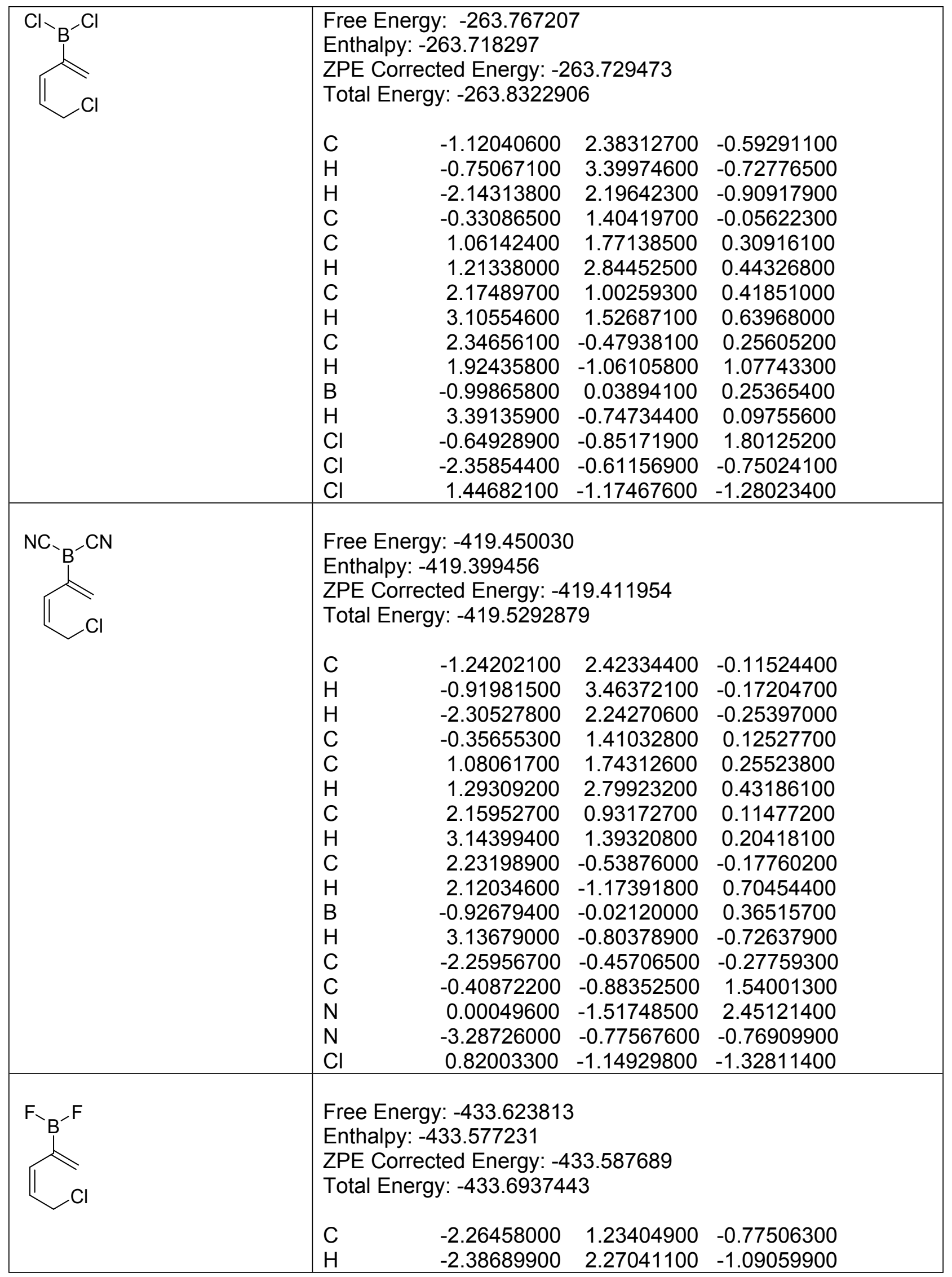




\begin{tabular}{|c|c|c|c|c|}
\hline & $\begin{array}{l}\mathrm{H} \\
\mathrm{C} \\
\mathrm{C} \\
\mathrm{H} \\
\mathrm{C} \\
\mathrm{H} \\
\mathrm{C} \\
\mathrm{H} \\
\mathrm{B} \\
\mathrm{H} \\
\mathrm{F} \\
\mathrm{F} \\
\mathrm{Cl}\end{array}$ & $\begin{array}{r}-3.07514700 \\
-1.14854100 \\
-0.07337000 \\
-0.38118600 \\
1.24120000 \\
1.87335900 \\
1.97205700 \\
1.64327000 \\
-1.14515600 \\
3.05251000 \\
-1.95877700 \\
-0.48220400 \\
1.68283000\end{array}$ & $\begin{array}{r}0.55335000 \\
0.81478300 \\
1.79317600 \\
2.83696400 \\
1.57661300 \\
2.45878600 \\
0.27345400 \\
-0.33604900 \\
-0.65197600 \\
0.41289200 \\
-1.59446800 \\
-1.07116600 \\
-0.88813100\end{array}$ & $\begin{array}{r}-1.02419300 \\
-0.10950500 \\
0.16730500 \\
0.07537200 \\
0.43420000 \\
0.53713700 \\
0.56959200 \\
1.41068000 \\
0.37863300 \\
0.58941200 \\
-0.19384500 \\
1.50376700 \\
-0.93526100\end{array}$ \\
\hline & $\begin{array}{l}\text { Fr } \\
\text { En } \\
\text { ZF } \\
\text { To } \\
\text { C } \\
\mathrm{H} \\
\mathrm{H} \\
\mathrm{C} \\
\mathrm{C} \\
\mathrm{H} \\
\mathrm{C} \\
\mathrm{H} \\
\mathrm{C} \\
\mathrm{H} \\
\mathrm{B} \\
\mathrm{H} \\
\mathrm{H} \\
\mathrm{H} \\
\mathrm{Cl}\end{array}$ & $\begin{array}{l}y:-234.96552 \\
234.924293 \\
\text { ted Energy: }-2 \\
y:-235.05060 \\
-2.75514200 \\
-3.41664800 \\
-3.18691400 \\
-1.46326000 \\
-0.94007600 \\
-1.66733000 \\
0.34568900 \\
0.57166700 \\
1.53379400 \\
1.51900400 \\
-0.66619700 \\
2.48025800 \\
-0.87273800 \\
-0.07821700 \\
1.62681600\end{array}$ & $\begin{array}{l}9 \\
34.933053 \\
3 \\
-0.38927100 \\
0.39937000 \\
-1.38630800 \\
-0.14124400 \\
1.22725400 \\
1.96318800 \\
1.65219700 \\
2.68492000 \\
0.85174100 \\
0.55812000 \\
-1.29615400 \\
1.32739200 \\
-2.42911100 \\
-1.09059800 \\
-0.86766300\end{array}$ & $\begin{array}{r} \\
-0.28512500 \\
-0.64934900 \\
-0.22170200 \\
0.08767600 \\
-0.10774100 \\
-0.46105300 \\
0.01670300 \\
-0.24895600 \\
0.45413200 \\
1.50459300 \\
0.78647600 \\
0.19574700 \\
0.47187100 \\
1.80665100 \\
-0.43082700\end{array}$ \\
\hline $\mathrm{H}_{2} \mathrm{~N}_{-}-\mathrm{NH}_{2}$ & $\begin{array}{l}\mathrm{C} \\
\mathrm{H} \\
\mathrm{H} \\
\mathrm{C} \\
\mathrm{C} \\
\mathrm{H} \\
\mathrm{C} \\
\mathrm{H} \\
\mathrm{C} \\
\mathrm{H} \\
\mathrm{B}\end{array}$ & $\begin{array}{r}-2.75159700 \\
-2.89388400 \\
-3.64282200 \\
-1.52556400 \\
-0.39248500 \\
-0.64278500 \\
0.88580600 \\
1.53956400 \\
1.51708400 \\
0.84727300 \\
-1.42372600 \\
\end{array}$ & $\begin{array}{l}\text { l5.723475 } \\
31 \\
1.24685700 \\
2.31832300 \\
0.62974100 \\
0.70907500 \\
1.66015400 \\
2.66731400 \\
1.50964900 \\
2.37960600 \\
0.30483300 \\
-0.53410100 \\
-0.87331300 \\
\end{array}$ & $\begin{array}{r}-0.45649000 \\
-0.60888900 \\
-0.55071000 \\
-0.18556700 \\
-0.11290500 \\
-0.45991700 \\
0.33772500 \\
0.27613600 \\
0.94104200 \\
1.10643400 \\
-0.05601300 \\
\end{array}$ \\
\hline
\end{tabular}




\begin{tabular}{|c|c|c|c|c|}
\hline & $\begin{array}{l}\mathrm{H} \\
\mathrm{N} \\
\mathrm{H} \\
\mathrm{H} \\
\mathrm{N} \\
\mathrm{H} \\
\mathrm{H} \\
\mathrm{Cl}\end{array}$ & $\begin{array}{r}2.10534300 \\
-2.35561700 \\
-3.01094900 \\
-2.41925000 \\
-0.45526700 \\
-0.35887000 \\
0.22461700 \\
2.86159500 \\
\end{array}$ & $\begin{array}{r}0.53732700 \\
-1.53927400 \\
-1.02011500 \\
-2.54552200 \\
-1.59280500 \\
-2.60060500 \\
-1.12734500 \\
-0.41158300 \\
\end{array}$ & $\begin{array}{r}1.82980800 \\
0.79713100 \\
1.36599600 \\
0.89043300 \\
-0.81041500 \\
-0.78941700 \\
-1.39863200 \\
-0.26064800 \\
\end{array}$ \\
\hline$\underbrace{\mathrm{CO}_{\mathrm{B}}^{-\mathrm{OH}}}_{\mathrm{Cl}}$ & $\begin{array}{l}\mathrm{C} \\
\mathrm{H} \\
\mathrm{H} \\
\mathrm{C} \\
\mathrm{C} \\
\mathrm{H} \\
\mathrm{C} \\
\mathrm{H} \\
\mathrm{C} \\
\mathrm{H} \\
\mathrm{B} \\
\mathrm{H} \\
\mathrm{O} \\
\mathrm{H} \\
\mathrm{O} \\
\mathrm{H} \\
\mathrm{Cl}\end{array}$ & $\begin{array}{r}-2.42800100 \\
-2.42098400 \\
-3.32705200 \\
-1.35051900 \\
-0.16970300 \\
-0.38444700 \\
1.12164000 \\
1.78291200 \\
1.77890900 \\
1.09039400 \\
-1.55532100 \\
2.59960000 \\
-2.56631300 \\
-2.80253800 \\
-0.80955900 \\
-0.95946100 \\
2.67595300\end{array}$ & $\begin{array}{l}\text { l } \\
85.490259 \\
1.46968500 \\
2.55102900 \\
0.94119400 \\
0.80573800 \\
1.64135500 \\
2.70950100 \\
1.36327500 \\
2.22381700 \\
0.04520700 \\
-0.76160300 \\
-0.73621200 \\
0.09834800 \\
-1.33425400 \\
-2.27423100 \\
-1.45158500 \\
-2.39798700 \\
-0.53375700\end{array}$ & $\begin{array}{r}-0.57287000 \\
-0.71521600 \\
-0.87559900 \\
-0.05398400 \\
0.27196800 \\
0.16903100 \\
0.60659900 \\
0.71217300 \\
0.81724300 \\
1.04093300 \\
0.14951700 \\
1.53304300 \\
-0.59315800 \\
-0.51489500 \\
1.08000400 \\
1.25097300 \\
-0.80332500\end{array}$ \\
\hline (c) & $\begin{array}{l}\text { Fre } \\
\text { En } \\
\text { ZP } \\
\text { To } \\
\text { Fre } \\
\text { C } \\
\mathrm{H} \\
\mathrm{H} \\
\mathrm{C} \\
\mathrm{C} \\
\mathrm{H} \\
\mathrm{C} \\
\mathrm{H} \\
\mathrm{C} \\
\mathrm{H} \\
\mathrm{B} \\
\mathrm{H} \\
\mathrm{Cl}\end{array}$ & $\begin{array}{l}y:-260.161916 \\
60.112271 \\
\text { ted Energy: }-2 \\
y:-260.224052 \\
-393.9227 \\
-1.50333600 \\
-2.29228700 \\
-1.12323900 \\
-0.68410900 \\
-1.24262500 \\
-0.53582400 \\
-2.59453800 \\
-2.78886600 \\
-3.71701800 \\
-3.80966200 \\
0.78759300 \\
-4.66856500 \\
-3.31945200\end{array}$ & $\begin{array}{l}60.123189 \\
24 \\
0.76860500 \\
0.60569400 \\
1.78633800 \\
-0.29946700 \\
-1.59415800 \\
-2.36920400 \\
-1.90584500 \\
-2.79907500 \\
-1.09980700 \\
-0.54477200 \\
-0.02363100 \\
-1.34222000 \\
1.08545900 \\
\end{array}$ & $\begin{array}{r}0.94114700 \\
1.66383100 \\
0.91486700 \\
0.48255600 \\
0.20957400 \\
-0.08233400 \\
0.00534900 \\
-0.58828400 \\
0.30743600 \\
1.23046700 \\
0.14426800 \\
-0.16269800 \\
-0.62866400\end{array}$ \\
\hline
\end{tabular}




\begin{tabular}{|c|c|c|c|c|}
\hline & $\begin{array}{l}\mathrm{Br} \\
\mathrm{Br}\end{array}$ & $\begin{array}{l}2.08753300 \\
1.51706500\end{array}$ & $\begin{array}{r}-1.49819400 \\
1.81569700\end{array}$ & $\begin{array}{r}-0.14412100 \\
0.01022700\end{array}$ \\
\hline 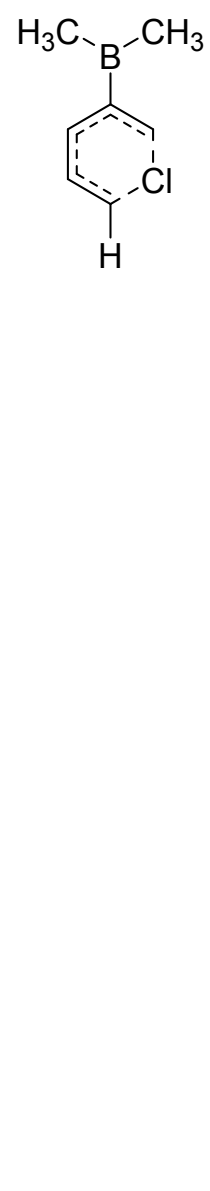 & $\begin{array}{l}\text { Fre } \\
\text { En } \\
\text { ZP } \\
\text { To } \\
\text { Fre } \\
\text { C } \\
\text { H } \\
\text { H } \\
\text { C } \\
\text { C } \\
\text { H } \\
\text { C } \\
\text { H } \\
\text { C } \\
\mathrm{H} \\
\text { B } \\
\mathrm{H} \\
\mathrm{Cl} \\
\mathrm{C} \\
\mathrm{H} \\
\mathrm{H} \\
\mathrm{H} \\
\mathrm{C} \\
\mathrm{H} \\
\mathrm{H} \\
\mathrm{H}\end{array}$ & $\begin{array}{r}0.17905400 \\
0.96659100 \\
-0.18466400 \\
-0.61373400 \\
-0.07376800 \\
-0.78809600 \\
1.28181500 \\
1.47067200 \\
2.40600800 \\
2.44458200 \\
-2.08654100 \\
3.37982600 \\
2.15736800 \\
-3.15319800 \\
-2.92993900 \\
-3.21680500 \\
-4.15722800 \\
-2.55184500 \\
-1.75824400 \\
-2.92329500 \\
-3.39194100\end{array}$ & $\begin{array}{l}13.495114 \\
45 \\
-0.76763500 \\
-0.54305500 \\
-1.79068400 \\
0.22617900 \\
1.49211300 \\
2.21096400 \\
1.82217000 \\
2.63555000 \\
1.10863400 \\
0.60922100 \\
-0.12492700 \\
1.33807700 \\
-1.25124100 \\
1.01923800 \\
1.98735600 \\
1.18479500 \\
0.70464700 \\
-1.63395800 \\
-2.32375900 \\
-1.99012900 \\
-1.72769600\end{array}$ & $\begin{array}{r}1.02710300 \\
1.73530700 \\
1.07749000 \\
0.41592900 \\
0.02890500 \\
-0.37311000 \\
-0.17804500 \\
-0.87936200 \\
0.27260600 \\
1.22870900 \\
0.00098300 \\
-0.15569500 \\
-0.46878500 \\
-0.26553500 \\
0.19990200 \\
-1.35574000 \\
0.05080800 \\
-0.14802000 \\
-0.46201800 \\
0.82917100 \\
-0.84868400\end{array}$ \\
\hline$\underbrace{C}_{-}$ & $\begin{array}{l}\text { Fre } \\
\text { En } \\
\text { ZP } \\
\text { To } \\
\text { Fre } \\
\text { C } \\
\mathrm{H} \\
\mathrm{H} \\
\mathrm{C} \\
\mathrm{C} \\
\mathrm{H} \\
\mathrm{C} \\
\mathrm{H} \\
\mathrm{C} \\
\mathrm{H} \\
\mathrm{B} \\
\mathrm{H} \\
\mathrm{Cl} \\
\mathrm{Cl}\end{array}$ & $\begin{array}{l}\text { : }-263.736697 \\
63.689700 \\
\text { ted Energy: }-2 \\
y:-263.80213 \subseteq \\
-394.0970 \\
0.73081000 \\
1.51319100 \\
0.32545500 \\
-0.05652100 \\
0.52190100 \\
-0.17109500 \\
1.88140800 \\
2.10028500 \\
2.98383100 \\
3.04941700 \\
-1.51989800 \\
3.94783700 \\
2.56716500 \\
-2.66128100\end{array}$ & $\begin{array}{l}63.700145 \\
97 \\
\\
-0.85127800 \\
-0.70948700 \\
-1.85928200 \\
0.23312800 \\
1.51961000 \\
2.30195900 \\
1.81181600 \\
2.70157000 \\
0.98592900 \\
0.42833500 \\
-0.01895400 \\
1.21075400 \\
-1.19473000 \\
1.34472600\end{array}$ & $\begin{array}{r}0.92775400 \\
1.66166800 \\
0.89416300 \\
0.45539100 \\
0.18960900 \\
-0.11620800 \\
0.00894500 \\
-0.58113900 \\
0.33028000 \\
1.25405600 \\
0.08530300 \\
-0.12280000 \\
-0.61555300 \\
-0.29769300\end{array}$ \\
\hline
\end{tabular}




\begin{tabular}{|c|c|c|c|c|}
\hline & $\mathrm{Cl}$ & -2.23142300 & -1.68966300 & 0.03747300 \\
\hline - & $\begin{array}{l}\text { Fre } \\
\text { En } \\
\text { ZP } \\
\text { Tot } \\
\text { Fre } \\
\text { C } \\
\mathrm{H} \\
\mathrm{H} \\
\mathrm{C} \\
\mathrm{C} \\
\mathrm{H} \\
\mathrm{C} \\
\mathrm{H} \\
\mathrm{C} \\
\mathrm{H} \\
\mathrm{B} \\
\mathrm{H} \\
\mathrm{C} \\
\mathrm{C} \\
\mathrm{N} \\
\mathrm{N} \\
\mathrm{Cl}\end{array}$ & $\begin{array}{l}y:-419.42002 \\
419.370635 \\
\text { ted Energy: }- \\
y y:-419.49919 \\
-392.7515 \\
\\
-0.56291000 \\
-1.30619200 \\
-0.15995000 \\
0.23133800 \\
-0.33765700 \\
0.36369500 \\
-1.69019700 \\
-1.91901400 \\
-2.79181400 \\
-2.86041100 \\
1.69169500 \\
-3.75648800 \\
2.65239700 \\
2.29203600 \\
3.38056200 \\
2.74666200 \\
-2.38057900\end{array}$ & $\begin{array}{l}19.382461 \\
35 \\
0.90334500 \\
0.79615300 \\
1.90993200 \\
-0.20194800 \\
-1.50642300 \\
-2.29348200 \\
-1.80783200 \\
-2.72123200 \\
-0.96006300 \\
-0.41731500 \\
0.04261900 \\
-1.19129200 \\
-1.12638100 \\
1.46091800 \\
-2.03213300 \\
2.55364700 \\
1.14610800\end{array}$ & $\begin{array}{r}0.86377300 \\
1.64550700 \\
0.77305700 \\
0.42752800 \\
0.20543200 \\
-0.06944000 \\
0.03802000 \\
-0.51002500 \\
0.34022000 \\
1.27427200 \\
0.08203700 \\
-0.10857100 \\
-0.21309400 \\
0.04495100 \\
-0.43860800 \\
0.01782000 \\
-0.63002600\end{array}$ \\
\hline 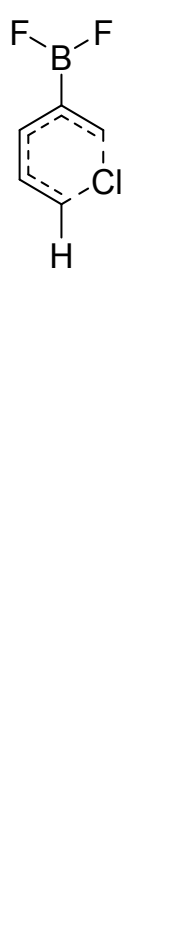 & $\begin{array}{l}\text { Fre } \\
\text { En } \\
\text { ZP } \\
\text { Tot } \\
\text { Fre } \\
\text { C } \\
\mathrm{H} \\
\mathrm{H} \\
\mathrm{C} \\
\mathrm{C} \\
\mathrm{H} \\
\mathrm{C} \\
\mathrm{H} \\
\mathrm{C} \\
\mathrm{H} \\
\mathrm{B} \\
\mathrm{H} \\
\mathrm{F} \\
\mathrm{F} \\
\mathrm{Cl}\end{array}$ & $\begin{array}{l}\text { y: }-433.58834 \\
433.543751 \\
\text { cted Energy: }- \\
y \mathrm{y}:-433.65848 \\
-392.0759 \\
0.12918600 \\
0.90214900 \\
-0.28102200 \\
-0.63475900 \\
-0.08898200 \\
-0.79625800 \\
1.26969000 \\
1.48990100 \\
2.37122700 \\
2.39965100 \\
-2.07157500 \\
3.35139700 \\
-2.61685600 \\
-2.91483100 \\
2.04700400\end{array}$ & $\begin{array}{l}\text { 33.553562 } \\
08 \\
-0.86581200 \\
-0.70864900 \\
-1.87351100 \\
0.19396000 \\
1.47872000 \\
2.21937300 \\
1.80056000 \\
2.66319300 \\
1.01662800 \\
0.48378900 \\
-0.09867100 \\
1.24070300 \\
-1.35553700 \\
0.88607400 \\
-1.23827700\end{array}$ & $\begin{array}{r}0.94450600 \\
1.68429100 \\
0.93078300 \\
0.40190600 \\
0.08986400 \\
-0.28364100 \\
-0.05890600 \\
-0.68728300 \\
0.34619600 \\
1.28572600 \\
-0.02525600 \\
-0.07059400 \\
0.04191000 \\
-0.47747300 \\
-0.53849000\end{array}$ \\
\hline & & $\begin{array}{l}\mathrm{y}:-234.93157 \\
234.891594\end{array}$ & & \\
\hline
\end{tabular}




\begin{tabular}{|c|c|c|c|c|}
\hline ו'- & $\begin{array}{l}\mathrm{C} \\
\mathrm{H} \\
\mathrm{H} \\
\mathrm{C} \\
\mathrm{C} \\
\mathrm{H} \\
\mathrm{C} \\
\mathrm{H} \\
\mathrm{C} \\
\mathrm{H} \\
\mathrm{B} \\
\mathrm{H} \\
\mathrm{H} \\
\mathrm{H} \\
\mathrm{Cl}\end{array}$ & $\begin{array}{r}-0.51516600 \\
0.11089400 \\
-0.69683600 \\
-1.41843700 \\
-1.09729800 \\
-1.90135600 \\
0.18102700 \\
0.29602900 \\
1.36115100 \\
1.37632500 \\
-2.73543300 \\
2.31997800 \\
-2.90657900 \\
-3.61330600 \\
1.62496200 \\
\end{array}$ & $\begin{array}{l}34.899920 \\
91 \\
-1.03140100 \\
-0.72975500 \\
-2.10267600 \\
-0.15053200 \\
1.21929900 \\
1.80418300 \\
1.80151100 \\
2.67808900 \\
1.26435700 \\
0.76309000 \\
-0.72747200 \\
1.68027500 \\
-1.90936700 \\
0.00475500 \\
-1.01003800 \\
\end{array}$ & $\begin{array}{r}0.83080000 \\
1.66267400 \\
0.77022200 \\
0.18036300 \\
-0.10175900 \\
-0.55076200 \\
-0.13725600 \\
-0.77473600 \\
0.42056500 \\
1.37773700 \\
-0.35776100 \\
0.11683000 \\
-0.40747000 \\
-0.71112100 \\
-0.40299100\end{array}$ \\
\hline$\underbrace{-\mathrm{Cl}_{2}}_{\mathrm{H}}$ & $\begin{array}{l}\mathrm{C} \\
\mathrm{H} \\
\mathrm{H} \\
\mathrm{C} \\
\mathrm{C} \\
\mathrm{H} \\
\mathrm{C} \\
\mathrm{H} \\
\mathrm{C} \\
\mathrm{H} \\
\mathrm{B} \\
\mathrm{H} \\
\mathrm{N} \\
\mathrm{H} \\
\mathrm{H} \\
\mathrm{N} \\
\mathrm{H} \\
\mathrm{H} \\
\mathrm{Cl}\end{array}$ & $\begin{array}{l}\text { y: }-345.71883 \\
345.671930 \\
\text { ted Energy: }-3 \\
y:-345.83758 \\
-335.6300 \\
\\
0.11993000 \\
0.94703200 \\
-0.25122600 \\
-0.66057900 \\
-0.14887000 \\
-0.87491000 \\
1.20508200 \\
1.37385400 \\
2.34310600 \\
2.38252500 \\
-2.11077800 \\
3.31430800 \\
-2.26208500 \\
-1.45586900 \\
-3.15199500 \\
-3.20557400 \\
-4.14057900 \\
-3.11494900 \\
2.15572400\end{array}$ & $\begin{array}{r}45.682963 \\
1 \\
-0.56435800 \\
-0.23273900 \\
-1.55680700 \\
0.29212600 \\
1.48473900 \\
2.09264900 \\
1.82468100 \\
2.52583700 \\
1.22639200 \\
0.81727700 \\
-0.18830100 \\
1.42119800 \\
-1.52119100 \\
-2.09993600 \\
-1.94077800 \\
0.72259700 \\
0.51221300 \\
1.62076000 \\
-1.30644100\end{array}$ & $\begin{array}{r}1.19393100 \\
1.80527400 \\
1.44078600 \\
0.40709200 \\
-0.18130600 \\
-0.72491100 \\
-0.41784500 \\
-1.23606800 \\
0.13583700 \\
1.13251000 \\
-0.02746000 \\
-0.31458800 \\
-0.50561000 \\
-0.70695700 \\
-0.74380100 \\
0.06842200 \\
-0.25789300 \\
0.52460800 \\
-0.26750600\end{array}$ \\
\hline
\end{tabular}




\begin{tabular}{|c|c|c|c|c|}
\hline (- & $\begin{array}{l}\mathrm{C} \\
\mathrm{H} \\
\mathrm{H} \\
\mathrm{C} \\
\mathrm{C} \\
\mathrm{H} \\
\mathrm{C} \\
\mathrm{H} \\
\mathrm{C} \\
\mathrm{H} \\
\mathrm{B} \\
\mathrm{H} \\
\mathrm{O} \\
\mathrm{H} \\
\mathrm{O} \\
\mathrm{H} \\
\mathrm{Cl}\end{array}$ & $\begin{array}{r}0.11932400 \\
0.93887300 \\
-0.30074700 \\
-0.63695600 \\
-0.09421300 \\
-0.81205800 \\
1.26659900 \\
1.46765600 \\
2.37878700 \\
2.39899000 \\
-2.09829300 \\
3.36026200 \\
-2.56746700 \\
-3.44778800 \\
-2.86118600 \\
-3.78813200 \\
2.11190500 \\
\end{array}$ & $\begin{array}{l} \\
95.456856 \\
95 \\
-0.85254500 \\
-0.70120600 \\
-1.85526100 \\
0.19777700 \\
1.47582100 \\
2.20024300 \\
1.81710800 \\
2.67587600 \\
1.06839200 \\
0.48284500 \\
-0.10270800 \\
1.31924300 \\
-1.40231200 \\
-1.69185500 \\
0.95062800 \\
0.85770000 \\
-1.25881700 \\
\end{array}$ & $\begin{array}{r}0.97625400 \\
1.66404900 \\
0.97154300 \\
0.42591200 \\
0.10402300 \\
-0.27999500 \\
-0.04823600 \\
-0.68933200 \\
0.36537100 \\
1.27146400 \\
-0.01793800 \\
-0.03230500 \\
0.11487300 \\
-0.17744900 \\
-0.50897700 \\
-0.78618000 \\
-0.56701300 \\
\end{array}$ \\
\hline - & $\begin{array}{l}\text { Fre } \\
\text { En } \\
\text { ZP } \\
\text { To } \\
\text { Fre } \\
\text { C } \\
\mathrm{H} \\
\mathrm{H} \\
\mathrm{C} \\
\mathrm{C} \\
\mathrm{H} \\
\mathrm{C} \\
\mathrm{H} \\
\mathrm{C} \\
\mathrm{H} \\
\mathrm{H} \\
\mathrm{B} \\
\mathrm{Cl} \\
\mathrm{Br} \\
\mathrm{Br}\end{array}$ & $\begin{array}{l}\mathrm{y}:-260.15393 \\
60.104128 \\
\text { ted Energy: }-2 \\
\mathrm{y}:-260.21227 \\
-1343.5195 \\
-1.15140700 \\
-1.62816300 \\
-0.70847400 \\
-0.43671000 \\
-1.20168000 \\
-0.69458200 \\
-2.58924600 \\
-3.06119200 \\
-3.35897200 \\
-3.66349000 \\
-2.34927200 \\
1.02658600 \\
-4.84698500 \\
1.92093500 \\
2.13046800\end{array}$ & $\begin{array}{l}60.114907 \\
33 \\
1.08573300 \\
0.99571700 \\
2.07010400 \\
-0.07274100 \\
-1.25362800 \\
-2.17264600 \\
-1.19306000 \\
-1.98555200 \\
-0.01470900 \\
0.26131300 \\
0.94718800 \\
0.01181900 \\
0.22980800 \\
1.74807400 \\
-1.61640300\end{array}$ & $\begin{array}{r}1.07802600 \\
2.05840300 \\
0.92286600 \\
0.59351000 \\
0.35482800 \\
0.07418800 \\
0.16962300 \\
-0.40715900 \\
0.45357500 \\
1.46089000 \\
0.42092500 \\
0.14022300 \\
-0.63553500 \\
-0.19058100 \\
-0.10440500\end{array}$ \\
\hline
\end{tabular}




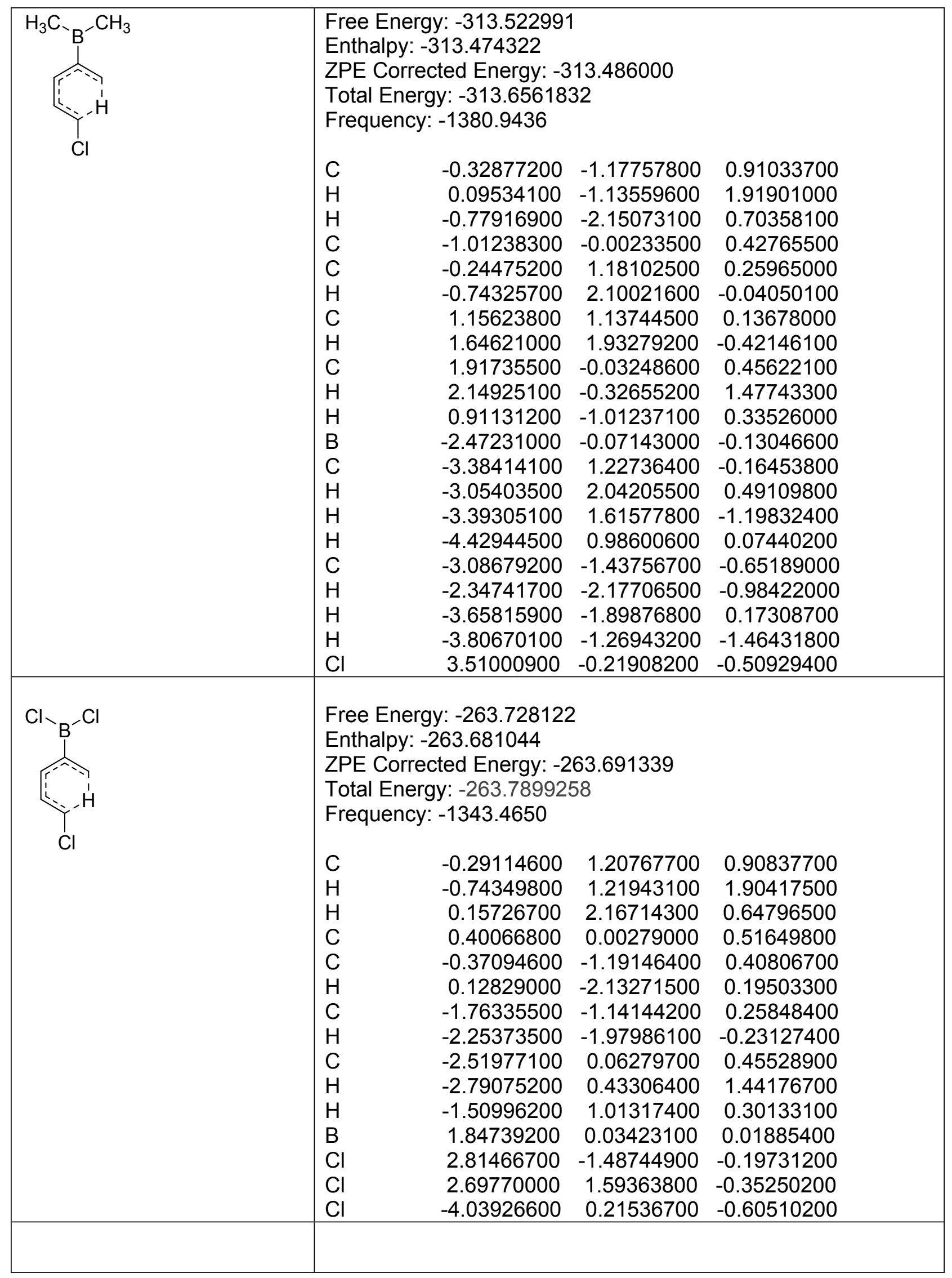




\begin{tabular}{|c|c|c|c|c|}
\hline Cll & $\begin{array}{l}\mathrm{C} \\
\mathrm{H} \\
\mathrm{H} \\
\mathrm{C} \\
\mathrm{C} \\
\mathrm{H} \\
\mathrm{C} \\
\mathrm{H} \\
\mathrm{C} \\
\mathrm{H} \\
\mathrm{H} \\
\mathrm{B} \\
\mathrm{C} \\
\mathrm{C} \\
\mathrm{N} \\
\mathrm{N} \\
\mathrm{Cl}\end{array}$ & $\begin{array}{c}y:-419.4102 \\
419.360611 \\
\text { cted Energy: } \\
y=-419.4854 \\
-1288.6167 \\
-0.09079000 \\
-0.51744000 \\
0.33504900 \\
0.60509100 \\
-0.15860900 \\
0.34915500 \\
-1.5478690 \\
-2.04352500 \\
-2.30763100 \\
-2.58280800 \\
-1.31230600 \\
2.04849600 \\
2.76534100 \\
2.85989300 \\
3.30559400 \\
3.47011400 \\
-3.80312500\end{array}$ & $\begin{array}{l}4 \\
19.372360 \\
76 \\
1.21216900 \\
1.23843400 \\
2.17366300 \\
0.00664100 \\
-1.19921300 \\
-2.13904100 \\
-1.15804900 \\
-2.00213300 \\
0.04866900 \\
0.40878300 \\
1.00422300 \\
0.04502400 \\
1.37669500 \\
-1.24978000 \\
2.40583200 \\
-2.25297500 \\
0.22342100 \\
\end{array}$ & $\begin{array}{r}0.88640000 \\
1.89336000 \\
0.59379200 \\
0.49312500 \\
0.40162600 \\
0.19943400 \\
0.25939700 \\
-0.21475200 \\
0.45740100 \\
1.44683300 \\
0.30216100 \\
0.00678100 \\
-0.28112600 \\
-0.18794200 \\
-0.50616100 \\
-0.33877800 \\
-0.61843800 \\
\end{array}$ \\
\hline - & $\begin{array}{l}\mathrm{C} \\
\mathrm{H} \\
\mathrm{H} \\
\mathrm{C} \\
\mathrm{C} \\
\mathrm{H} \\
\mathrm{C} \\
\mathrm{H} \\
\mathrm{C} \\
\mathrm{H} \\
\mathrm{H} \\
\mathrm{B} \\
\mathrm{Cl} \\
\mathrm{F} \\
\mathrm{F}\end{array}$ & $\begin{array}{c}y:-433.5787 \\
433.534130 \\
\text { cted Energy: } \\
y \mathrm{y}:-433.6453 \\
-1380.3438 \\
\\
-0.38791900 \\
0.03475800 \\
-0.8477240 \\
-1.04547500 \\
-0.28176300 \\
-0.77752200 \\
1.11835600 \\
1.62725000 \\
1.86306600 \\
2.07778000 \\
0.86135400 \\
-2.46813800 \\
3.44379900 \\
-3.16399200 \\
-3.14460500\end{array}$ & $\begin{array}{l}3 \\
76.543769 \\
-1.31748500 \\
-1.46281700 \\
-2.22831200 \\
-0.06549100 \\
1.13049700 \\
2.08820400 \\
1.09350900 \\
1.97723500 \\
-0.12797700 \\
-0.61617800 \\
-1.05576800 \\
-0.02668400 \\
-0.15890900 \\
1.14616300 \\
-1.16236500 \\
\end{array}$ & $\begin{array}{r}0.65636300 \\
1.65458700 \\
0.26934800 \\
0.38926800 \\
0.44721100 \\
0.30971000 \\
0.35422200 \\
-0.02446600 \\
0.44774900 \\
1.39595600 \\
0.12873700 \\
-0.16680400 \\
-0.53343700 \\
-0.31533300 \\
-0.52914500 \\
\end{array}$ \\
\hline$\overbrace{-1}^{-H}$ & \multicolumn{4}{|c|}{$\begin{array}{l}\text { Free Energy: }-234.922154 \\
\text { Enthalpy: -234.882263 } \\
\text { ZPE Corrected Energy: }-234.890442 \\
\text { Total Energy: }-235.0030561 \\
\text { Frequency: }-1350.8324\end{array}$} \\
\hline
\end{tabular}




\begin{tabular}{|c|c|c|c|c|}
\hline & $\begin{array}{l}\mathrm{C} \\
\mathrm{H} \\
\mathrm{H} \\
\mathrm{C} \\
\mathrm{C} \\
\mathrm{H} \\
\mathrm{C} \\
\mathrm{H} \\
\mathrm{C} \\
\mathrm{H} \\
\mathrm{H} \\
\mathrm{B} \\
\mathrm{H} \\
\mathrm{H} \\
\mathrm{Cl}\end{array}$ & $\begin{array}{r}-1.28006300 \\
-0.99824400 \\
-1.67788500 \\
-1.91647400 \\
-1.15942800 \\
-1.64622400 \\
0.24407600 \\
0.78950900 \\
0.97577500 \\
1.08600600 \\
0.01489800 \\
-3.26941100 \\
-3.68402000 \\
-3.93058100 \\
2.65942800 \\
\end{array}$ & $\begin{array}{r}-1.34203500 \\
-1.55854300 \\
-2.22290300 \\
-0.07286600 \\
1.11453800 \\
2.07927400 \\
1.08418600 \\
1.98077000 \\
-0.14202300 \\
-0.66445400 \\
-1.05627200 \\
-0.00333000 \\
-0.94761900 \\
0.99263100 \\
-0.14335500 \\
\end{array}$ & $\begin{array}{r}0.33101500 \\
1.36678900 \\
-0.17838100 \\
0.05812700 \\
0.27283500 \\
0.14356400 \\
0.31530900 \\
0.02842800 \\
0.44440500 \\
1.39212400 \\
-0.02183600 \\
-0.66369100 \\
-1.26904000 \\
-0.61233500 \\
-0.35653000 \\
\end{array}$ \\
\hline $\mathrm{H}_{2} \mathrm{~N}-\mathrm{B}^{-}-\mathrm{NH}_{2}$ & $\begin{array}{l}\mathrm{C} \\
\mathrm{H} \\
\mathrm{H} \\
\mathrm{C} \\
\mathrm{C} \\
\mathrm{H} \\
\mathrm{C} \\
\mathrm{H} \\
\mathrm{C} \\
\mathrm{H} \\
\mathrm{H} \\
\mathrm{B} \\
\mathrm{Cl} \\
\mathrm{N} \\
\mathrm{H} \\
\mathrm{H} \\
\mathrm{N} \\
\mathrm{H} \\
\mathrm{H}\end{array}$ & $\begin{array}{l}y:-345.708329 \\
345.661962 \\
\text { ted Energy: }-3 \\
y:-345.82411 \\
-1437.0107 \\
\\
0.36867900 \\
-0.07588500 \\
0.84468300 \\
1.04621400 \\
0.28108200 \\
0.77292300 \\
-1.12451800 \\
-1.61395300 \\
-1.88866500 \\
-2.11002200 \\
-0.88231900 \\
2.52040900 \\
-3.51288200 \\
2.94938300 \\
2.31702800 \\
3.88947900 \\
3.39511400 \\
4.34404000 \\
3.12274700\end{array}$ & $\begin{array}{l}\text { L5.672669 } \\
56 \\
\\
1.06844700 \\
0.89221300 \\
2.05140700 \\
-0.03268600 \\
-1.18136800 \\
-2.03959300 \\
-1.13082500 \\
-1.85427200 \\
-0.01289000 \\
0.16189500 \\
0.98056100 \\
0.10214600 \\
0.24547900 \\
1.33027300 \\
2.10475300 \\
1.49011500 \\
-1.02162200 \\
-1.03088600 \\
-1.86468600\end{array}$ & $\begin{array}{r} \\
1.03691300 \\
2.02166800 \\
0.99486400 \\
0.40741100 \\
0.08750800 \\
-0.36672200 \\
-0.00393000 \\
-0.65346100 \\
0.45709400 \\
1.50781500 \\
0.44206400 \\
-0.15189500 \\
-0.45306400 \\
-0.73814000 \\
-0.88851200 \\
-1.07750100 \\
-0.04406700 \\
-0.39656900 \\
0.44340500 \\
\end{array}$ \\
\hline 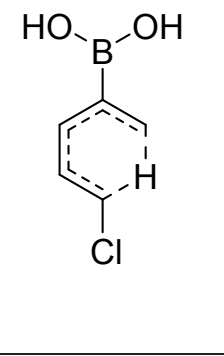 & $\begin{array}{l}\mathrm{Fr} \\
\mathrm{Er} \\
\mathrm{ZF} \\
\mathrm{Tc} \\
\mathrm{Fr}\end{array}$ & $\begin{array}{l}y:-385.485182 \\
385.439834 \\
\text { ted Energy: }-3 \\
y:-385.57574 \\
-1421.2235 \\
-0.37527000 \\
0.05999600\end{array}$ & $\begin{array}{l}85.449955 \\
18 \\
-1.35880100 \\
-1.54920300\end{array}$ & $\begin{array}{r} \\
0.60146200 \\
1.58705300\end{array}$ \\
\hline
\end{tabular}




\begin{tabular}{|c|c|c|c|c|}
\hline & $\begin{array}{l}\mathrm{H} \\
\mathrm{C} \\
\mathrm{C} \\
\mathrm{H} \\
\mathrm{C} \\
\mathrm{H} \\
\mathrm{C} \\
\mathrm{H} \\
\mathrm{H} \\
\mathrm{B} \\
\mathrm{Cl} \\
\mathrm{O} \\
\mathrm{H} \\
\mathrm{O} \\
\mathrm{H}\end{array}$ & $\begin{array}{c}-0.82836400 \\
-1.04272400 \\
-0.27510400 \\
-0.78197500 \\
1.12716000 \\
1.62298500 \\
1.88189400 \\
2.10014600 \\
0.87624000 \\
-2.49828600 \\
3.48470400 \\
-3.26763700 \\
-2.91777900 \\
-3.07931400 \\
-3.99990300\end{array}$ & $\begin{array}{r}-2.26891500 \\
-0.10111300 \\
1.08601300 \\
2.04095000 \\
1.06061000 \\
1.96038800 \\
-0.15467200 \\
-0.67715800 \\
-1.07321900 \\
-0.00289600 \\
-0.12077000 \\
-1.10007300 \\
-2.00230800 \\
1.24427100 \\
1.29123300\end{array}$ & $\begin{array}{c}0.19446500 \\
0.39345200 \\
0.48911500 \\
0.38180400 \\
0.38072600 \\
0.02173400 \\
0.42872500 \\
1.35794700 \\
0.07739000 \\
-0.15890000 \\
-0.53515100 \\
-0.52526100 \\
-0.43290300 \\
-0.28191400 \\
-0.59889400\end{array}$ \\
\hline$\overbrace{\mathrm{Cl}}^{\mathrm{H}_{3} \mathrm{C}{ }_{-}{ }^{-}-\mathrm{CH}_{3}}$ & $\begin{array}{l}\text { Fre } \\
\text { En } \\
\text { ZP } \\
\text { Tot } \\
\text { C } \\
\text { H } \\
\text { C } \\
\text { C } \\
\text { H } \\
\text { C } \\
\text { H } \\
\text { C } \\
\text { H } \\
\mathrm{H} \\
\mathrm{B} \\
\mathrm{C} \\
\mathrm{H} \\
\mathrm{H} \\
\mathrm{H} \\
\mathrm{C} \\
\mathrm{H} \\
\mathrm{H} \\
\mathrm{H} \\
\mathrm{H} \\
\mathrm{Cl}\end{array}$ & $\begin{array}{c}:-313.577209 \\
313.525781 \\
\text { ted Energy: }-3 \\
y:-313.713369 \\
0.10991600 \\
1.02322300 \\
0.17455200 \\
-0.99204000 \\
-0.91687900 \\
-2.35849900 \\
-2.50273400 \\
-3.44907900 \\
-3.35477200 \\
-0.75838400 \\
1.57575400 \\
1.73328600 \\
1.97889100 \\
0.86036900 \\
2.59464400 \\
2.89305600 \\
2.77589700 \\
3.75909200 \\
3.14668500 \\
-4.45810400 \\
-0.04081400\end{array}$ & $\begin{array}{l} \\
13.538416 \\
95 \\
-1.09844300 \\
-1.39799800 \\
0.29865800 \\
0.97757000 \\
2.00312500 \\
0.46971400 \\
-0.56906500 \\
1.23249100 \\
2.27198400 \\
-1.30279800 \\
0.94861500 \\
2.45867700 \\
2.46738000 \\
3.10533200 \\
2.92222700 \\
0.07835200 \\
-0.95035600 \\
0.53221900 \\
0.00070400 \\
0.83556800 \\
-2.42138400\end{array}$ & $\begin{array}{r} \\
0.89093800 \\
1.40109700 \\
0.33334700 \\
0.06165800 \\
-0.30124000 \\
0.17231600 \\
0.46249300 \\
-0.09866200 \\
-0.41021100 \\
1.51834000 \\
0.03698100 \\
-0.41884400 \\
-1.49514000 \\
-0.27387400 \\
0.08414900 \\
0.19060700 \\
-0.17613800 \\
-0.30671200 \\
1.26364200 \\
-0.02055500 \\
-0.51287800\end{array}$ \\
\hline$\underbrace{\mathrm{Cl}}_{-\mathrm{B}^{-C l}}$ & \multicolumn{4}{|c|}{$\begin{array}{l}\text { Free Energy: }-262.004212 \\
\text { Enthalpy: -261.953144 } \\
\text { ZPE Corrected Energy: }-261.964626 \\
\text { Total Energy: -262.0671888 }\end{array}$} \\
\hline
\end{tabular}




\begin{tabular}{|c|c|c|c|c|}
\hline & $\begin{array}{l}\mathrm{C} \\
\mathrm{H} \\
\mathrm{C} \\
\mathrm{H} \\
\mathrm{C} \\
\mathrm{H} \\
\mathrm{H} \\
\mathrm{B} \\
\mathrm{H} \\
\mathrm{Cl} \\
\mathrm{Cl} \\
\mathrm{Cl}\end{array}$ & $\begin{array}{r}-1.11045000 \\
-0.73904600 \\
-2.55677700 \\
-2.99173500 \\
-3.37527000 \\
-2.98180200 \\
-1.47394900 \\
1.29804700 \\
-4.45441400 \\
-1.33147700 \\
1.92453800 \\
2.54103700 \\
\end{array}$ & $\begin{array}{r}1.08334100 \\
2.06425900 \\
0.98159500 \\
0.01251600 \\
2.05278100 \\
3.03683700 \\
-1.27205200 \\
0.32908300 \\
1.96807900 \\
-2.32586900 \\
1.94577500 \\
-0.96581300 \\
\end{array}$ & $\begin{array}{r}0.15008900 \\
-0.14757600 \\
0.29117900 \\
0.52343700 \\
0.11632200 \\
-0.13444400 \\
1.49867900 \\
0.08575100 \\
0.21541300 \\
-0.67839800 \\
-0.45901100 \\
0.33363400 \\
\end{array}$ \\
\hline$\overbrace{\mathrm{Cl}}^{\mathrm{NC}}$ & $\begin{array}{l}\mathrm{C} \\
\mathrm{H} \\
\mathrm{C} \\
\mathrm{C} \\
\mathrm{H} \\
\mathrm{C} \\
\mathrm{H} \\
\mathrm{C} \\
\mathrm{H} \\
\mathrm{H} \\
\mathrm{B} \\
\mathrm{H} \\
\mathrm{Cl} \\
\mathrm{C} \\
\mathrm{C} \\
\mathrm{N} \\
\mathrm{N}\end{array}$ & $\begin{array}{r}-0.39383800 \\
0.46362500 \\
-0.01702800 \\
-0.98515800 \\
-0.65692200 \\
-2.41992400 \\
-2.81070700 \\
-3.28271200 \\
-2.93029200 \\
-1.17386600 \\
1.44763400 \\
-4.35681700 \\
-1.13185900 \\
1.86978300 \\
2.57708500 \\
2.18329000 \\
3.44230600\end{array}$ & $\begin{array}{l}17.644897 \\
1 \\
-1.32672700 \\
-1.92128100 \\
0.06990800 \\
1.05052300 \\
2.05230200 \\
0.88324800 \\
-0.10474400 \\
1.92372700 \\
2.92410700 \\
-1.36259300 \\
0.42790200 \\
1.79542900 \\
-2.32032500 \\
1.84904000 \\
-0.60215100 \\
2.94454100 \\
-1.39634200\end{array}$ & $\begin{array}{r}0.76077300 \\
1.07099500 \\
0.33612900 \\
0.16967700 \\
-0.11186100 \\
0.31899600 \\
0.54830400 \\
0.15703200 \\
-0.09026800 \\
1.52269900 \\
0.08521500 \\
0.26356700 \\
-0.70027600 \\
-0.33697300 \\
0.26165500 \\
-0.65760700 \\
0.41067000\end{array}$ \\
\hline$\approx$ & $\begin{array}{l}\mathrm{C} \\
\mathrm{H} \\
\mathrm{C} \\
\mathrm{C} \\
\mathrm{H} \\
\mathrm{C} \\
\mathrm{H} \\
\mathrm{C} \\
\mathrm{H} \\
\mathrm{H}\end{array}$ & $\begin{array}{l}:-234.976527 \\
34.933602 \\
\text { ted Energy: }-2 \\
y:-235.06010 \\
0.24215900 \\
1.20436700 \\
0.22228700 \\
-0.94684200 \\
-0.87422400 \\
-2.30055700 \\
-2.42432600 \\
-3.40132000 \\
-3.32013500 \\
-0.56746100\end{array}$ & $\begin{array}{l}34.942837 \\
6 \\
-1.09424900 \\
-1.35376700 \\
0.29314600 \\
0.96571000 \\
1.98061000 \\
0.45117200 \\
-0.57621800 \\
1.20046800 \\
2.22755000 \\
-1.30123000\end{array}$ & $\begin{array}{r}0.87532900 \\
1.31416600 \\
0.30072600 \\
0.03035900 \\
-0.36374000 \\
0.20030500 \\
0.53559900 \\
-0.06820300 \\
-0.42123800 \\
1.57538600 \\
\end{array}$ \\
\hline
\end{tabular}




\begin{tabular}{|c|c|c|c|c|}
\hline & $\begin{array}{l}\mathrm{B} \\
\mathrm{H} \\
\mathrm{Cl} \\
\mathrm{F} \\
\mathrm{F}\end{array}$ & $\begin{array}{r}1.55783000 \\
-4.40569400 \\
0.00864300 \\
1.63716200 \\
2.75806400 \\
\end{array}$ & $\begin{array}{r}0.97745900 \\
0.80332700 \\
-2.41744500 \\
2.23890500 \\
0.37570800 \\
\end{array}$ & $\begin{array}{r}0.00438500 \\
0.05406000 \\
-0.49785000 \\
-0.52950900 \\
0.27575300 \\
\end{array}$ \\
\hline $\mathrm{HO}_{-}-\mathrm{OH}$ & $\begin{array}{l}\text { Fre } \\
\text { Ent } \\
\text { ZPE } \\
\text { Tot } \\
\text { C } \\
\text { H } \\
\text { C } \\
\text { C } \\
\text { H } \\
\text { C } \\
\mathrm{H} \\
\mathrm{C} \\
\mathrm{H} \\
\mathrm{H} \\
\mathrm{B} \\
\mathrm{H} \\
\mathrm{Cl} \\
\mathrm{O} \\
\mathrm{H} \\
\mathrm{O} \\
\mathrm{H} \\
\end{array}$ & $\begin{array}{l}:-385.538525 \\
85.490323 \\
\text { ted Energy: }-3 \\
y:-385.631376 \\
0.20072200 \\
1.15948600 \\
0.20786800 \\
-0.94777500 \\
-0.85090700 \\
-2.31320500 \\
-2.45111700 \\
-3.40800800 \\
-3.31715100 \\
-0.62179300 \\
1.58353400 \\
-4.41612400 \\
-0.03289700 \\
1.60669200 \\
2.42652200 \\
2.72406600 \\
3.62897800 \\
\end{array}$ & $\begin{array}{r}\text { r } \\
85.501581 \\
-1.09792000 \\
-1.37144300 \\
0.29144800 \\
0.98296000 \\
1.99859300 \\
0.48965800 \\
-0.54049100 \\
1.25123200 \\
2.28130500 \\
-1.31036200 \\
0.95423700 \\
0.86260400 \\
-2.42220700 \\
2.21122400 \\
2.69362400 \\
0.25416900 \\
0.56509700 \\
\end{array}$ & $\begin{array}{r}0.86589800 \\
1.30088300 \\
0.30157800 \\
0.04252300 \\
-0.34260700 \\
0.20875100 \\
0.53269400 \\
-0.04656900 \\
-0.38861100 \\
1.54940500 \\
0.01407300 \\
0.07428600 \\
-0.52516900 \\
-0.57836500 \\
-0.77805500 \\
0.38605800 \\
0.21488700 \\
\end{array}$ \\
\hline $\mathrm{Br} \backslash_{\mathrm{B}^{-}}-\mathrm{Br}$ & $\begin{array}{l}\text { Fre } \\
\text { Ent } \\
\text { ZPE } \\
\text { Tot } \\
\text { C } \\
\mathrm{H} \\
\mathrm{C} \\
\mathrm{C} \\
\mathrm{H} \\
\mathrm{C} \\
\mathrm{H} \\
\mathrm{C} \\
\mathrm{H} \\
\mathrm{H} \\
\mathrm{B} \\
\mathrm{H} \\
\mathrm{Cl} \\
\mathrm{Br} \\
\mathrm{Br}\end{array}$ & $\begin{array}{l}\text { d: }-260.207886 \\
60.155616 \\
\text { ted Energy: }-2 \\
\text { y: }-260.269462 \\
\\
1.43106200 \\
0.67320300 \\
0.87302500 \\
1.72481200 \\
1.29200600 \\
3.17565400 \\
3.67702200 \\
3.91996100 \\
3.45977500 \\
2.26075500 \\
-0.63823800 \\
5.00321900 \\
2.17889500 \\
-1.44872000 \\
-1.89308300\end{array}$ & $\begin{array}{l}60.167360 \\
2.1 \\
-1.40955700 \\
-2.11283400 \\
-0.04931300 \\
1.03450100 \\
2.01138600 \\
1.01657500 \\
0.06406100 \\
2.15058300 \\
3.12269800 \\
-1.37753100 \\
0.13114000 \\
2.13048700 \\
-2.26447100 \\
1.89391800 \\
-1.39262400\end{array}$ & $\begin{array}{r}-0.69992200 \\
-1.03706500 \\
-0.36124500 \\
-0.25705200 \\
-0.04156100 \\
-0.38522700 \\
-0.53793600 \\
-0.29220000 \\
-0.12095100 \\
-1.40638500 \\
-0.12735700 \\
-0.37982500 \\
0.84580100 \\
0.29518000 \\
-0.24501500\end{array}$ \\
\hline
\end{tabular}




\begin{tabular}{|c|c|c|c|c|}
\hline$\underbrace{\mathrm{H}_{2} \mathrm{~N}^{\mathrm{B}^{-}} \mathrm{NH}_{2}}_{\mathrm{Cl}}$ & $\begin{array}{l}\text { Fre } \\
\text { Ent } \\
\text { ZPE } \\
\text { Tot } \\
\text { C } \\
\mathrm{H} \\
\mathrm{C} \\
\mathrm{C} \\
\mathrm{H} \\
\mathrm{C} \\
\mathrm{H} \\
\mathrm{C} \\
\mathrm{H} \\
\mathrm{H} \\
\mathrm{B} \\
\mathrm{H} \\
\mathrm{Cl} \\
\mathrm{N} \\
\mathrm{H} \\
\mathrm{H} \\
\mathrm{N} \\
\mathrm{H} \\
\mathrm{H}\end{array}$ & $\begin{array}{l}:-345.76554 \\
45.717207 \\
\text { ted Energy: }- \\
y:-345.88507 \\
0.31282200 \\
1.14036000 \\
0.00581700 \\
-1.27750900 \\
-1.44330800 \\
-2.48205000 \\
-2.38052400 \\
-3.71484200 \\
-3.87221600 \\
-0.53890800 \\
1.20434700 \\
-4.59201300 \\
0.97992300 \\
0.98263000 \\
0.11969300 \\
1.67993200 \\
2.47964200 \\
3.30451500 \\
2.60071800\end{array}$ & $\begin{array}{l}\text { L5.728767 } \\
-0.95971800 \\
-0.88795200 \\
0.33841800 \\
0.65615200 \\
1.62196800 \\
-0.16401800 \\
-1.16302400 \\
0.25190800 \\
1.23553500 \\
-1.46656900 \\
1.33393600 \\
-0.38097400 \\
-2.28746600 \\
2.74213100 \\
3.12215600 \\
3.43723300 \\
0.79857800 \\
1.36558100 \\
-0.18812100\end{array}$ & $\begin{array}{r}0.96893000 \\
1.67414600 \\
0.28401400 \\
-0.08115700 \\
-0.56375700 \\
0.06251500 \\
0.48414300 \\
-0.32481800 \\
-0.76552600 \\
1.42031900 \\
-0.00532200 \\
-0.21703300 \\
-0.29468700 \\
0.09913600 \\
0.46411000 \\
-0.13664300 \\
-0.34755000 \\
-0.49846500 \\
-0.54301500\end{array}$ \\
\hline & $\begin{array}{l}\text { Fre } \\
\text { Ent } \\
\text { ZPE } \\
\text { Tot } \\
\mathrm{C} \\
\mathrm{H} \\
\mathrm{H} \\
\mathrm{C} \\
\mathrm{C} \\
\mathrm{H} \\
\mathrm{C} \\
\mathrm{H} \\
\mathrm{C} \\
\mathrm{H} \\
\mathrm{H} \\
\mathrm{B} \\
\mathrm{Br} \\
\mathrm{Br} \\
\mathrm{Cl}\end{array}$ & $\begin{array}{r}0.26037100 \\
-0.25852500 \\
-0.25805300 \\
0.22125900 \\
1.36854500 \\
1.25956100 \\
2.74364600 \\
2.94373900 \\
3.78349000 \\
3.69923000 \\
1.27789200 \\
-1.14985500 \\
-1.36306200 \\
-2.82656000 \\
5.49755100\end{array}$ & $\begin{array}{l} \\
60.166886 \\
71 \\
-2.28120000 \\
-2.68330300 \\
-2.68325400 \\
-0.75730500 \\
0.00904400 \\
1.09363500 \\
-0.47283500 \\
-1.54013300 \\
0.39365000 \\
1.47483900 \\
-2.68158800 \\
-0.05269800 \\
1.92343200 \\
-1.10542200 \\
-0.15854000 \\
\end{array}$ & $\begin{array}{r}0.00007100 \\
0.87933400 \\
-0.87949800 \\
0.00009700 \\
0.00011100 \\
0.00012400 \\
0.00000100 \\
-0.00018600 \\
0.00011000 \\
0.00025400 \\
0.00037800 \\
0.00005000 \\
-0.00000900 \\
-0.00003500 \\
-0.00008500 \\
\end{array}$ \\
\hline $\mathrm{H}_{3} \mathrm{C}_{\mathrm{B}^{-}}-\mathrm{CH}_{3}$ & \multicolumn{4}{|c|}{$\begin{array}{l}\text { Free Energy: }-313.574772 \\
\text { Enthalpy: }-313.522242 \\
\text { ZPE Corrected Energy: }-313.535273 \\
\text { Total Energy: }-313.7091534\end{array}$} \\
\hline
\end{tabular}




\begin{tabular}{|c|c|c|c|c|}
\hline & $\begin{array}{l}\mathrm{C} \\
\mathrm{H} \\
\mathrm{H} \\
\mathrm{C} \\
\mathrm{C} \\
\mathrm{H} \\
\mathrm{C} \\
\mathrm{H} \\
\mathrm{C} \\
\mathrm{H} \\
\mathrm{H} \\
\mathrm{B} \\
\mathrm{C} \\
\mathrm{H} \\
\mathrm{H} \\
\mathrm{H} \\
\mathrm{C} \\
\mathrm{H} \\
\mathrm{H} \\
\mathrm{H} \\
\mathrm{Cl}\end{array}$ & $\begin{array}{r}1.23949800 \\
1.53574500 \\
1.96869000 \\
1.24918400 \\
0.08437400 \\
0.15908400 \\
-1.28289900 \\
-1.44346700 \\
-2.35497800 \\
-2.32111300 \\
0.26624900 \\
2.63549700 \\
2.77734100 \\
3.61257500 \\
3.06072500 \\
1.88948900 \\
3.96946000 \\
4.83101200 \\
4.21586000 \\
3.87115500 \\
-4.05384500 \\
\end{array}$ & $\begin{array}{r}1.93325600 \\
2.34682000 \\
2.30789500 \\
0.40882100 \\
-0.32032700 \\
-1.40769100 \\
0.20022900 \\
1.27497200 \\
-0.62145800 \\
-1.70491400 \\
2.36449100 \\
-0.33747000 \\
-1.91569700 \\
-2.18785400 \\
-2.28934000 \\
-2.47063600 \\
0.51170900 \\
-0.09172200 \\
0.93890900 \\
1.36819400 \\
0.00347200 \\
\end{array}$ & $\begin{array}{r}-0.09142500 \\
0.88427500 \\
-0.82106300 \\
-0.05512900 \\
-0.03150100 \\
-0.00723800 \\
-0.01908900 \\
-0.03183300 \\
0.01519800 \\
0.03555600 \\
-0.34938100 \\
0.00883300 \\
-0.06723600 \\
-0.73013200 \\
0.93207400 \\
-0.39048100 \\
0.14921800 \\
0.46092800 \\
-0.83993800 \\
0.83036200 \\
0.03426400 \\
\end{array}$ \\
\hline ( & $\begin{array}{l}\text { Fre } \\
\text { En } \\
\text { ZP } \\
\text { To } \\
\text { C } \\
\mathrm{H} \\
\mathrm{H} \\
\mathrm{C} \\
\mathrm{C} \\
\mathrm{H} \\
\mathrm{C} \\
\mathrm{H} \\
\mathrm{C} \\
\mathrm{H} \\
\mathrm{H} \\
\mathrm{B} \\
\mathrm{N} \\
\mathrm{H} \\
\mathrm{H} \\
\mathrm{N} \\
\mathrm{H} \\
\mathrm{H} \\
\mathrm{Cl}\end{array}$ & $\begin{array}{l}y:-345.76168 \\
345.712303 \\
\text { ted Energy: }-3 \\
y:-345.87945 \\
1.27753500 \\
1.90652800 \\
1.70537900 \\
1.27037300 \\
0.11632400 \\
0.20024200 \\
-1.25686200 \\
-1.42077500 \\
-2.33080500 \\
-2.30640500 \\
0.28403100 \\
2.67291200 \\
3.79974200 \\
3.72367800 \\
4.73068400 \\
2.80057700 \\
3.65305100 \\
2.04725500 \\
-4.03234400 \\
\end{array}$ & $\begin{array}{l}\text { l. } \\
45.724269 \\
11 \\
1.88749000 \\
2.12460400 \\
2.43148500 \\
0.38837800 \\
-0.33199700 \\
-1.39753300 \\
0.17201400 \\
1.22466100 \\
-0.62517100 \\
-1.68498800 \\
2.31013400 \\
-0.33802200 \\
0.39499500 \\
1.34992800 \\
0.00455600 \\
-1.70962300 \\
-2.24530000 \\
-2.20882400 \\
0.00232400 \\
\end{array}$ & $\begin{array}{r}0.35979800 \\
1.22873000 \\
-0.49726800 \\
0.09610300 \\
-0.05256100 \\
-0.27695500 \\
0.02923700 \\
0.24645900 \\
-0.15479400 \\
-0.38029800 \\
0.54065900 \\
-0.03201800 \\
-0.51684000 \\
-0.83905700 \\
-0.59194300 \\
0.34824600 \\
0.24250800 \\
0.80134800 \\
-0.04709600 \\
\end{array}$ \\
\hline $\mathrm{Cl}^{-}$ & \multicolumn{4}{|c|}{$\begin{array}{l}\text { Free Energy: }-234.974502 \\
\text { Enthalpy: }-234.931009 \\
\text { ZPE Corrected Energy: }-234.940598 \\
\text { Total Energy: }-235.0568902\end{array}$} \\
\hline
\end{tabular}




\begin{tabular}{|c|c|c|c|c|}
\hline & $\begin{array}{l}\mathrm{C} \\
\mathrm{H} \\
\mathrm{H} \\
\mathrm{C} \\
\mathrm{C} \\
\mathrm{H} \\
\mathrm{C} \\
\mathrm{H} \\
\mathrm{C} \\
\mathrm{H} \\
\mathrm{H} \\
\mathrm{B} \\
\mathrm{H} \\
\mathrm{H} \\
\mathrm{Cl}\end{array}$ & $\begin{array}{r}2.31601000 \\
1.84334000 \\
3.36796900 \\
2.19067700 \\
0.95285700 \\
0.91714000 \\
-0.33338500 \\
-0.34235100 \\
-1.50369000 \\
-1.60424100 \\
1.84523300 \\
3.43461500 \\
3.30345100 \\
4.54106300 \\
-3.10467600\end{array}$ & $\begin{array}{r}1.47596000 \\
1.92667400 \\
1.77672400 \\
-0.04595700 \\
-0.65809900 \\
-1.74859100 \\
0.03266400 \\
1.11967100 \\
-0.64453200 \\
-1.72431700 \\
1.92624500 \\
-0.95755200 \\
-2.14783000 \\
-0.50269500 \\
0.18833300\end{array}$ & $\begin{array}{r}0.00002800 \\
0.88493900 \\
0.00103400 \\
0.00012700 \\
-0.00002100 \\
0.00006400 \\
-0.00007100 \\
-0.00011500 \\
-0.00000900 \\
0.00010800 \\
-0.88613900 \\
-0.00002400 \\
0.00001700 \\
-0.00021800 \\
0.00000600\end{array}$ \\
\hline & $\begin{array}{l}\text { Fre } \\
\text { En } \\
\text { ZP } \\
\text { To } \\
\text { C } \\
\text { H } \\
\text { H } \\
\text { C } \\
\text { C } \\
\text { H } \\
\text { C } \\
\text { H } \\
\text { C } \\
\text { H } \\
\text { H } \\
\text { B } \\
\text { Cl } \\
\text { F } \\
\text { F }\end{array}$ & $\begin{array}{l}y:-433.634497 \\
\text { t33.586262 } \\
\text { ted Energy: }-4 \\
y:-433.702474 \\
-1.35621100 \\
-1.90653800 \\
-1.90658200 \\
-1.28886200 \\
-0.12831000 \\
-0.21125200 \\
1.23129700 \\
1.38347900 \\
2.30582900 \\
2.26909500 \\
-0.37259300 \\
-2.61791200 \\
3.99672600 \\
-2.69687500 \\
-3.82455400\end{array}$ & $\begin{array}{l}33.597305 \\
42 \\
1.92178100 \\
2.28261900 \\
2.28259300 \\
0.40443000 \\
-0.32882500 \\
-1.41676400 \\
0.20129000 \\
1.27705700 \\
-0.61907700 \\
-1.70284900 \\
2.40077000 \\
-0.35789400 \\
0.00641100 \\
-1.72912100 \\
0.29350700\end{array}$ & $\begin{array}{r} \\
-0.00001800 \\
-0.87954800 \\
0.87950100 \\
-0.00002900 \\
-0.00002900 \\
0.00001100 \\
-0.00002000 \\
-0.00002800 \\
0.00000300 \\
0.00003300 \\
0.00001900 \\
0.00000000 \\
0.00001500 \\
-0.00002000 \\
0.00005400\end{array}$ \\
\hline $\mathrm{NC}_{\mathrm{B}^{-}}-\mathrm{CN}$ & $\begin{array}{l}\text { Fre } \\
\text { En } \\
\text { ZP } \\
\text { To } \\
\text { C } \\
\mathrm{H} \\
\mathrm{H} \\
\mathrm{C} \\
\mathrm{C} \\
\mathrm{H} \\
\mathrm{C} \\
\mathrm{H}\end{array}$ & $\begin{array}{l}y:-419.46465 \\
419.411635 \\
\text { ted Energy: }-4 \\
y:-419.541561 \\
-0.78972300 \\
-0.25804200 \\
-1.78511300 \\
-0.86845800 \\
0.28200400 \\
0.18146100 \\
1.63829100 \\
1.79741100\end{array}$ & $\begin{array}{l}0 \\
19.424720 \\
3 \\
-2.07077100 \\
-2.44631700 \\
-2.52273500 \\
-0.54871600 \\
0.22562200 \\
1.31156600 \\
-0.29181400 \\
-1.36660900 \\
\end{array}$ & $\begin{array}{r}0.00057300 \\
0.88587200 \\
0.00194200 \\
0.00032700 \\
-0.00003600 \\
-0.00035100 \\
-0.00002300 \\
0.00031000 \\
\end{array}$ \\
\hline
\end{tabular}




\begin{tabular}{|c|c|c|c|c|}
\hline & $\begin{array}{l}\mathrm{C} \\
\mathrm{H} \\
\mathrm{H} \\
\mathrm{B} \\
\mathrm{C} \\
\mathrm{C} \\
\mathrm{N} \\
\mathrm{N} \\
\mathrm{Cl}\end{array}$ & $\begin{array}{r}2.70484500 \\
2.64591800 \\
-0.26038700 \\
-2.21542200 \\
-3.54936900 \\
-2.31193100 \\
-2.37320400 \\
-4.57055200 \\
4.39577600 \\
\end{array}$ & $\begin{array}{r}0.54546400 \\
1.62883000 \\
-2.44658600 \\
0.17559000 \\
-0.59313300 \\
1.71541000 \\
2.89778700 \\
-1.19250100 \\
-0.05091000 \\
\end{array}$ & $\begin{array}{c}-0.00028200 \\
-0.00057000 \\
-0.88602300 \\
0.00044100 \\
0.00071500 \\
0.00021800 \\
0.00011900 \\
-0.00150800 \\
-0.00015400 \\
\end{array}$ \\
\hline & $\begin{array}{l}\text { Fre } \\
\text { En } \\
\text { ZP } \\
\text { Tot } \\
\text { C } \\
\mathrm{H} \\
\mathrm{H} \\
\mathrm{C} \\
\mathrm{C} \\
\mathrm{H} \\
\mathrm{C} \\
\mathrm{H} \\
\mathrm{C} \\
\mathrm{H} \\
\mathrm{H} \\
\mathrm{B} \\
\mathrm{Cl} \\
\mathrm{Cl} \\
\mathrm{Cl}\end{array}$ & $\begin{array}{l}y:-263.782696 \\
263.732132 \\
\text { ted Energy: }-26 \\
y:-263.846048 \\
-0.61062400 \\
-1.13487400 \\
-1.13562800 \\
-0.63109700 \\
0.51595900 \\
0.40703100 \\
1.88957900 \\
2.08373500 \\
2.93322800 \\
2.85373200 \\
0.40151300 \\
-1.99302800 \\
4.64477000 \\
-3.53949200 \\
-2.16955100\end{array}$ & $\begin{array}{l}63.743803 \\
33 \\
-2.10692900 \\
-2.50169300 \\
-2.50199000 \\
-0.58404700 \\
0.18037300 \\
1.26520700 \\
-0.30643000 \\
-1.37494100 \\
0.55506900 \\
1.63663400 \\
-2.52095200 \\
0.12760600 \\
-0.00467800 \\
-0.82071800 \\
1.93901200\end{array}$ & $\begin{array}{r}0.00034200 \\
0.88002200 \\
-0.87872600 \\
0.00018700 \\
0.00022300 \\
0.00029900 \\
0.00011900 \\
0.00008700 \\
0.00001500 \\
0.00005200 \\
0.00003100 \\
0.00001400 \\
-0.00019700 \\
-0.00031900 \\
0.00009500\end{array}$ \\
\hline & $\begin{array}{l}\text { Fre } \\
\text { En } \\
\text { ZP } \\
\text { Tot } \\
\mathrm{C} \\
\mathrm{H} \\
\mathrm{H} \\
\mathrm{C} \\
\mathrm{C} \\
\mathrm{H} \\
\mathrm{C} \\
\mathrm{H} \\
\mathrm{C} \\
\mathrm{H} \\
\mathrm{B} \\
\mathrm{H} \\
\mathrm{Br} \\
\mathrm{Br}\end{array}$ & $\begin{array}{r}-0.03256700 \\
0.53840800 \\
-1.10399400 \\
0.57337400 \\
2.03916700 \\
2.55508100 \\
2.80067200 \\
3.87273000 \\
2.34579900 \\
2.49790300 \\
-0.27201300 \\
2.81443900 \\
-2.08762400 \\
0.25182100 \\
\end{array}$ & $\begin{array}{l}45.056462 \\
22 \\
-2.07507200 \\
-2.80187100 \\
-2.05046500 \\
-1.24498700 \\
-1.37092400 \\
-1.93162500 \\
-0.92319000 \\
-1.12037700 \\
-0.19442200 \\
0.89039500 \\
-0.12024700 \\
-0.59179000 \\
-0.46445700 \\
1.79091800 \\
\end{array}$ & $\begin{array}{r}-1.82411300 \\
-2.40233400 \\
-2.00568900 \\
-0.92735800 \\
-0.73369100 \\
-1.51548000 \\
0.29339900 \\
0.26998100 \\
1.52371700 \\
1.46090800 \\
-0.25348900 \\
2.43038400 \\
0.44952600 \\
-0.52066500 \\
\end{array}$ \\
\hline
\end{tabular}




\begin{tabular}{|c|c|c|c|c|}
\hline & $\mathrm{F}$ & 0.89777300 & -0.37417900 & 1.72531000 \\
\hline $\mathrm{H}_{3} \mathrm{C}_{\mathrm{B}}-\mathrm{CH}_{3}$ & $\begin{array}{l}\text { Fre } \\
\text { En } \\
\text { ZP } \\
\text { To } \\
\text { C } \\
\text { H } \\
\text { H } \\
\text { C } \\
\text { C } \\
\text { H } \\
\text { C } \\
\text { H } \\
\text { C } \\
\text { H } \\
\text { B } \\
\text { H } \\
\text { F } \\
\text { C } \\
\text { H } \\
\mathrm{H} \\
\mathrm{H} \\
\mathrm{C} \\
\mathrm{H} \\
\mathrm{H} \\
\mathrm{H}\end{array}$ & $\begin{array}{r}-1.52172000 \\
-1.27722800 \\
-2.57728700 \\
-0.56646100 \\
0.83797800 \\
0.96563600 \\
1.97857900 \\
2.93565300 \\
2.10341500 \\
2.19044600 \\
-1.00415500 \\
2.93975600 \\
0.90485500 \\
-0.70119000 \\
-1.60372800 \\
-0.53455300 \\
0.13125100 \\
-2.02959000 \\
-2.19638200 \\
-3.00487500 \\
-1.69767300\end{array}$ & $\begin{array}{l}98.429690 \\
43 \\
2.02294500 \\
3.08701400 \\
1.78108800 \\
1.05992800 \\
1.51779100 \\
2.59750500 \\
0.78458700 \\
1.29900900 \\
-0.69369200 \\
-1.27361000 \\
-0.45609600 \\
-0.93017100 \\
-1.22273200 \\
-1.17752100 \\
-1.07439600 \\
-2.25561200 \\
-0.74311300 \\
-1.09695900 \\
-2.16811500 \\
-0.58932600 \\
-0.94767700 \\
\end{array}$ & $\begin{array}{r}-0.13220600 \\
-0.14104800 \\
-0.24029400 \\
0.01644600 \\
0.13343600 \\
0.25168000 \\
0.07120400 \\
0.16451500 \\
-0.16049000 \\
0.76747900 \\
0.19257800 \\
-0.82782300 \\
-0.83292800 \\
1.57652300 \\
2.20361400 \\
1.44855300 \\
2.14497300 \\
-0.83216300 \\
-0.66452200 \\
-0.74214200 \\
-1.86802200\end{array}$ \\
\hline & $\begin{array}{l}\text { Fre } \\
\text { En } \\
\text { ZP } \\
\text { To } \\
\\
\mathrm{C} \\
\mathrm{H} \\
\mathrm{H} \\
\mathrm{C} \\
\mathrm{C} \\
\mathrm{H} \\
\mathrm{C} \\
\mathrm{H} \\
\mathrm{C} \\
\mathrm{H} \\
\mathrm{B} \\
\mathrm{H} \\
\mathrm{F} \\
\mathrm{Cl} \\
\mathrm{Cl}\end{array}$ & $\begin{array}{r}-0.47435100 \\
0.08758400 \\
-1.55406700 \\
0.14893300 \\
1.62820200 \\
2.09750800 \\
2.45463200 \\
3.52987400 \\
2.06672600 \\
2.03276900 \\
-0.71549900 \\
2.70685100 \\
0.70123800 \\
-0.51459900 \\
-2.22534300 \\
\end{array}$ & $\begin{array}{l}3 \\
11.633984 \\
2.51684700 \\
3.43814600 \\
2.61829000 \\
1.30868600 \\
1.25979400 \\
2.15721500 \\
0.26285500 \\
0.39009800 \\
-1.03235700 \\
-1.87605300 \\
0.01539800 \\
-1.27099900 \\
-0.93796900 \\
-1.28743600 \\
-0.06473600 \\
\end{array}$ & $\begin{array}{r}0.33197300 \\
0.48680700 \\
0.25598800 \\
0.23906700 \\
0.32655500 \\
0.73336600 \\
-0.07168600 \\
0.05469600 \\
-0.72327900 \\
-0.02331500 \\
0.16250700 \\
-1.57890900 \\
-1.28162500 \\
1.43829900 \\
-0.83961300 \\
\end{array}$ \\
\hline & \multicolumn{4}{|c|}{$y:-504.3541$} \\
\hline
\end{tabular}




\begin{tabular}{|c|c|c|c|c|}
\hline $\mathrm{NC}_{\mathrm{B}^{-}}-\mathrm{CN}$ & $\begin{array}{l}\text { Er } \\
\text { ZF } \\
\text { Tc } \\
\text { C } \\
\mathrm{H} \\
\mathrm{H} \\
\mathrm{C} \\
\mathrm{C} \\
\mathrm{H} \\
\mathrm{C} \\
\mathrm{H} \\
\mathrm{C} \\
\mathrm{H} \\
\mathrm{B} \\
\mathrm{H} \\
\mathrm{F} \\
\mathrm{C} \\
\mathrm{C} \\
\mathrm{N} \\
\mathrm{N}\end{array}$ & $\begin{array}{c}504.305006 \\
\text { cted Energy: }-5 \\
\text { gy: }-504.43569 \\
-0.65545000 \\
-0.14472300 \\
-1.74252800 \\
0.03147800 \\
1.51055500 \\
1.99101500 \\
2.31335100 \\
3.39482600 \\
1.87620700 \\
1.97069200 \\
-0.73064100 \\
2.35110000 \\
0.41450600 \\
-2.10965100 \\
-0.41255900 \\
-0.14794700 \\
-3.16938500 \\
\end{array}$ & $\begin{array}{l}04.317104 \\
27 \\
2.41747900 \\
3.35809400 \\
2.45028800 \\
1.27028900 \\
1.29131200 \\
2.17293700 \\
0.34381300 \\
0.47349500 \\
-0.90478700 \\
-1.81732000 \\
-0.09294600 \\
-1.02994900 \\
-0.82437500 \\
-0.14196800 \\
-1.24756600 \\
-2.11463100 \\
-0.15606600 \\
\end{array}$ & $\begin{array}{r}0.59868000 \\
0.80725300 \\
0.60826400 \\
0.34593500 \\
0.30797600 \\
0.73420300 \\
-0.23429600 \\
-0.20524900 \\
-0.94274600 \\
-0.34312800 \\
0.18887100 \\
-1.92031400 \\
-1.27499700 \\
-0.50876700 \\
1.18175000 \\
1.94085800 \\
-1.03251700 \\
\end{array}$ \\
\hline & $\begin{array}{l}\mathrm{C} \\
\mathrm{H} \\
\mathrm{H} \\
\mathrm{C} \\
\mathrm{C} \\
\mathrm{H} \\
\mathrm{C} \\
\mathrm{H} \\
\mathrm{C} \\
\mathrm{H} \\
\mathrm{B} \\
\mathrm{H} \\
\mathrm{F} \\
\mathrm{F} \\
\mathrm{F}\end{array}$ & $\begin{array}{l}y:-518.52537 \\
518.481101 \\
\text { cted Energy: }-5 \\
\text { gy: }-518.59826 \\
-1.78096100 \\
-1.70225000 \\
-2.76624000 \\
-0.70442000 \\
0.62409900 \\
0.62398100 \\
1.82658600 \\
2.73390800 \\
2.06703600 \\
2.14155000 \\
-0.90523100 \\
2.93874200 \\
0.92599200 \\
-1.86483700 \\
-0.35421900\end{array}$ & $\begin{array}{l}3 \\
18.491100 \\
72 \\
1.73178700 \\
2.81668800 \\
1.30581600 \\
0.95404900 \\
1.57139400 \\
2.64406600 \\
0.95001600 \\
1.53027300 \\
-0.49485400 \\
-1.14145800 \\
-0.57340500 \\
-0.62467500 \\
-1.03825800 \\
-1.27152500 \\
-1.23888800\end{array}$ & $\begin{array}{r}-0.26323400 \\
-0.34025400 \\
-0.43950400 \\
0.03152400 \\
0.23924900 \\
0.43945800 \\
0.16452000 \\
0.33141200 \\
-0.17403200 \\
0.70782500 \\
0.22393100 \\
-0.82274000 \\
-0.94771400 \\
-0.46272600 \\
1.30110500\end{array}$ \\
\hline & \multicolumn{4}{|c|}{$\begin{array}{l}\text { Free Energy: }-319.866566 \\
\text { Enthalpy: }-319.827010 \\
\text { ZPE Corrected Energy: }-319.835305 \\
\text { Total Energy: }-319.9543673\end{array}$} \\
\hline
\end{tabular}




\begin{tabular}{|c|c|c|c|c|}
\hline & $\begin{array}{l}\mathrm{C} \\
\mathrm{C} \\
\mathrm{H} \\
\mathrm{C} \\
\mathrm{H} \\
\mathrm{C} \\
\mathrm{H} \\
\mathrm{B} \\
\mathrm{H} \\
\mathrm{F} \\
\mathrm{H} \\
\mathrm{H}\end{array}$ & $\begin{array}{r}1.18032800 \\
0.37960900 \\
0.93705300 \\
-0.97501500 \\
-1.46449300 \\
-1.91553000 \\
-2.22370600 \\
0.51095700 \\
-2.78827900 \\
-1.24442000 \\
0.82306900 \\
0.01892600 \\
\end{array}$ & $\begin{array}{r}0.02569500 \\
-1.21715000 \\
-2.15364900 \\
-1.29519000 \\
-2.26793900 \\
-0.12467500 \\
0.17517200 \\
1.40705700 \\
-0.26626500 \\
1.08906200 \\
2.39548200 \\
1.53466900 \\
\end{array}$ & $\begin{array}{r}0.05435600 \\
0.03885200 \\
-0.03865800 \\
0.06755500 \\
0.03285600 \\
0.10085200 \\
1.10937700 \\
0.41145300 \\
-0.54371900 \\
-0.45246400 \\
-0.18177300 \\
1.49844700 \\
\end{array}$ \\
\hline $\mathrm{H}_{2} \mathrm{~N}_{-}{ }^{-}-\mathrm{NH}_{2}$ & $\begin{array}{l}\text { Fr } \\
\text { Er } \\
\text { ZF } \\
\text { To } \\
\text { C } \\
\text { H } \\
\text { H } \\
\text { C } \\
\text { C } \\
\text { H } \\
\text { C } \\
\text { H } \\
\text { C } \\
\mathrm{H} \\
\text { B } \\
\mathrm{H} \\
\mathrm{F} \\
\mathrm{N} \\
\mathrm{H} \\
\mathrm{H} \\
\mathrm{N} \\
\mathrm{H} \\
\mathrm{H}\end{array}$ & $\begin{array}{l}y:-430.657842 \\
30.610260 \\
\text { ted Energy: }-4 \\
\text { y: }-430.77907 \\
-2.10782700 \\
-2.01987200 \\
-3.09106800 \\
-1.05234500 \\
0.24293700 \\
0.18232500 \\
1.48323400 \\
2.32894000 \\
1.87736200 \\
1.16209600 \\
-1.27928200 \\
2.08475800 \\
3.14489400 \\
-0.96417700 \\
-0.65790300 \\
-1.10168600 \\
-1.81096300 \\
-2.04734900 \\
-1.98206800\end{array}$ & $\begin{array}{l}30.621501 \\
71 \\
1.66213000 \\
2.74975900 \\
1.25583400 \\
0.85998200 \\
1.51071200 \\
2.58289700 \\
0.95872800 \\
1.59607700 \\
-0.45993700 \\
-0.99230900 \\
-0.70718900 \\
-1.04414900 \\
-0.42632000 \\
-1.36471900 \\
-0.84220300 \\
-2.35415400 \\
-1.41140700 \\
-2.39610100 \\
-0.93962800 \\
\end{array}$ & $\begin{array}{r} \\
-0.32730700 \\
-0.35786000 \\
-0.55725300 \\
-0.00811300 \\
0.30372700 \\
0.50947800 \\
0.34929300 \\
0.60850500 \\
0.04823800 \\
-0.58119400 \\
0.10331500 \\
0.95354100 \\
-0.69114600 \\
1.33086100 \\
2.14122200 \\
1.49630600 \\
-1.01617700 \\
-1.01239600 \\
-1.89442300\end{array}$ \\
\hline & $\begin{array}{l}\mathrm{C} \\
\mathrm{H} \\
\mathrm{H} \\
\mathrm{C} \\
\mathrm{C} \\
\mathrm{H} \\
\mathrm{C} \\
\mathrm{H}\end{array}$ & $\begin{array}{l}y:-470.42478 \\
770.379114 \\
\text { ted Energy: }-4 \\
y:-470.52088 \\
-1.79117700 \\
-1.67312100 \\
-2.78881800 \\
-0.74015200 \\
0.59308500 \\
0.57721400 \\
1.81987300 \\
2.68932700\end{array}$ & $\begin{array}{l}35 \\
70.389786 \\
91 \\
1.77253300 \\
2.85413300 \\
1.37688200 \\
0.95363100 \\
1.56543700 \\
2.64980700 \\
0.98572600 \\
1.62987800\end{array}$ & $\begin{array}{r}-0.23773000 \\
-0.32053500 \\
-0.41014200 \\
0.04622800 \\
0.23285000 \\
0.37271500 \\
0.19851200 \\
0.33442700\end{array}$ \\
\hline
\end{tabular}




\begin{tabular}{|c|c|c|c|c|}
\hline & $\begin{array}{l}\mathrm{C} \\
\mathrm{H} \\
\mathrm{B} \\
\mathrm{H} \\
\mathrm{F} \\
\mathrm{O} \\
\mathrm{H} \\
\mathrm{O} \\
\mathrm{H}\end{array}$ & $\begin{array}{r}2.14714800 \\
2.00507700 \\
-1.03279200 \\
3.16084000 \\
1.25330500 \\
-2.02746200 \\
-2.28654800 \\
-0.39851100 \\
-0.56463000 \\
\end{array}$ & $\begin{array}{l}-0.46062500 \\
-1.07360100 \\
-0.57265200 \\
-0.57860200 \\
-1.05186300 \\
-1.14006400 \\
-2.07491400 \\
-1.25431300 \\
-2.19875500 \\
\end{array}$ & $\begin{array}{r}-0.04141700 \\
0.85492100 \\
0.23453300 \\
-0.43937300 \\
-1.05279000 \\
-0.55003400 \\
-0.47820900 \\
1.27014200 \\
1.43712600 \\
\end{array}$ \\
\hline$\stackrel{H}{H}$ & $\begin{array}{l}\mathrm{C} \\
\mathrm{H} \\
\mathrm{H} \\
\mathrm{C} \\
\mathrm{C} \\
\mathrm{H} \\
\mathrm{C} \\
\mathrm{H} \\
\mathrm{C} \\
\mathrm{H} \\
\mathrm{H} \\
\mathrm{H} \\
\mathrm{F}\end{array}$ & $\begin{array}{l}y:-294.444922 \\
294.405879 \\
\text { ted Energy: }-2 \\
y:-294.520496 \\
-2.66266800 \\
-3.26059200 \\
-3.20797200 \\
-1.30671200 \\
-0.55259300 \\
-1.16677700 \\
0.79263200 \\
1.17015100 \\
1.91021900 \\
2.53802400 \\
2.53843000 \\
-0.73871600 \\
1.44913200 \\
\end{array}$ & \begin{tabular}{l}
\multicolumn{1}{l}{} \\
94.413482 \\
64 \\
-0.35681400 \\
0.55409200 \\
-1.29739400 \\
-0.32247600 \\
0.93846100 \\
1.84204000 \\
1.11968600 \\
2.14495500 \\
0.11322500 \\
0.22439900 \\
0.22458700 \\
-1.24732300 \\
-1.26642800 \\
\end{tabular} & $\begin{array}{r}0.00003300 \\
-0.00004400 \\
0.00011900 \\
0.00003400 \\
-0.00006800 \\
-0.00015000 \\
-0.00002800 \\
-0.00008300 \\
0.00013100 \\
0.89551800 \\
-0.89494500 \\
0.00011700 \\
-0.00012600 \\
\end{array}$ \\
\hline & $\begin{array}{l}\mathrm{C} \\
\mathrm{H} \\
\mathrm{H} \\
\mathrm{C} \\
\mathrm{C} \\
\mathrm{H} \\
\mathrm{C} \\
\mathrm{H} \\
\mathrm{C} \\
\mathrm{H} \\
\mathrm{H} \\
\mathrm{B} \\
\mathrm{O} \\
\mathrm{H} \\
\mathrm{O} \\
\mathrm{H}\end{array}$ & $\begin{array}{l}y:-470.438250 \\
70.390147 \\
\text { ted Energy: }-47 \\
0.81919700 \\
1.37280800 \\
1.37545200 \\
0.75534800 \\
-0.40765700 \\
-0.32091700 \\
-1.76689100 \\
-1.92969400 \\
-2.84865600 \\
-2.86452900 \\
-0.16543000 \\
2.10462900 \\
3.27920300 \\
4.16439700 \\
2.08793400 \\
2.89500300 \\
\end{array}$ & $\begin{array}{r}7 \\
70.401501 \\
1.93138700 \\
2.29073400 \\
2.29070100 \\
0.41443000 \\
-0.30758600 \\
-1.39467200 \\
0.22754400 \\
1.30249700 \\
-0.57637600 \\
-1.66213500 \\
2.41064900 \\
-0.35891600 \\
0.38673800 \\
-0.01670300 \\
-1.75174500 \\
-2.29373200 \\
\end{array}$ & $\begin{array}{r}0.00010400 \\
0.87807200 \\
-0.87615800 \\
0.00006000 \\
0.00004600 \\
-0.00005000 \\
-0.00000100 \\
0.00006600 \\
-0.00007100 \\
-0.00008900 \\
-0.00134200 \\
-0.00002100 \\
-0.00023400 \\
-0.00020000 \\
0.00014300 \\
0.00014100\end{array}$ \\
\hline
\end{tabular}




\begin{tabular}{|c|c|c|c|c|}
\hline & $\mathrm{F}$ & -4.14393000 & -0.03878800 & -0.00004800 \\
\hline 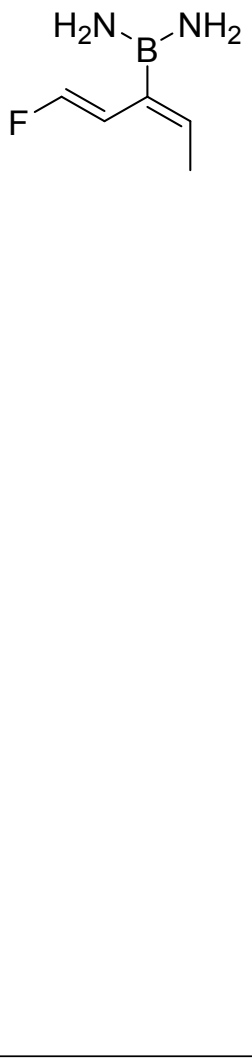 & $\begin{array}{l}\mathrm{C} \\
\mathrm{H} \\
\mathrm{H} \\
\mathrm{C} \\
\mathrm{C} \\
\mathrm{H} \\
\mathrm{C} \\
\mathrm{H} \\
\mathrm{C} \\
\mathrm{H} \\
\mathrm{H} \\
\mathrm{B} \\
\mathrm{N} \\
\mathrm{H} \\
\mathrm{H} \\
\mathrm{N} \\
\mathrm{H} \\
\mathrm{H} \\
\mathrm{F}\end{array}$ & $\begin{array}{l}\text { y: }-430.66308 \\
430.614977 \\
\text { ted Energy: } \\
y:-430.78308 \\
\\
0.77032700 \\
1.40400300 \\
1.19888300 \\
0.74821300 \\
-0.41437900 \\
-0.34137400 \\
-1.78089500 \\
-1.95057900 \\
-2.86263500 \\
-2.88286000 \\
-0.21929300 \\
2.14187400 \\
3.28092200 \\
3.21756700 \\
4.20811900 \\
2.25259200 \\
3.09926000 \\
1.49240000 \\
-4.15932100 \\
\end{array}$ & $\begin{array}{l}3 \\
62 \\
130.626629 \\
1.89329000 \\
2.12966400 \\
2.42990800 \\
0.39280600 \\
-0.31303300 \\
-1.38068000 \\
0.20631600 \\
1.25922000 \\
-0.57482600 \\
-1.63723300 \\
2.32461500 \\
-0.34941300 \\
0.37262200 \\
1.32979100 \\
-0.02756800 \\
-1.72494400 \\
-2.26974400 \\
-2.21736700 \\
-0.03940100 \\
\end{array}$ & $\begin{array}{r}0.35032600 \\
1.21608900 \\
-0.51119200 \\
0.09404900 \\
-0.05354300 \\
-0.27291500 \\
0.02169500 \\
0.23391700 \\
-0.16378200 \\
-0.38711000 \\
0.53270400 \\
-0.02782900 \\
-0.50140100 \\
-0.81969800 \\
-0.57082600 \\
0.34560200 \\
0.24001100 \\
0.79454100 \\
-0.07980600 \\
\end{array}$ \\
\hline & $\begin{array}{l}\mathrm{C} \\
\mathrm{H} \\
\mathrm{H} \\
\mathrm{C} \\
\mathrm{C} \\
\mathrm{H} \\
\mathrm{C} \\
\mathrm{H} \\
\mathrm{C} \\
\mathrm{H} \\
\mathrm{H} \\
\mathrm{B} \\
\mathrm{H} \\
\mathrm{H} \\
\mathrm{F}\end{array}$ & $\begin{array}{l}\text { y: }-319.87666 \\
319.834370 \\
\text { cted Energy: }- \\
y y:-319.96126 \\
1.85066400 \\
1.40229300 \\
2.91681600 \\
1.64587000 \\
0.37646000 \\
0.28419000 \\
-0.86945900 \\
-0.83852700 \\
-2.07209400 \\
-2.26481000 \\
1.40303400 \\
2.83755400 \\
2.64213100 \\
3.96751700 \\
-3.25434100\end{array}$ & $\begin{array}{l}19.843663 \\
46 \\
1.43432000 \\
1.90874300 \\
1.68016800 \\
-0.07862500 \\
-0.62224800 \\
-1.70956900 \\
0.13455700 \\
1.22123400 \\
-0.47751300 \\
-1.54672800 \\
1.90853500 \\
-1.05538400 \\
-2.23716600 \\
-0.66221200 \\
0.26344200\end{array}$ & $\begin{array}{r} \\
-0.00001600 \\
0.88527800 \\
0.00039400 \\
0.00004300 \\
0.00000600 \\
-0.00002200 \\
0.00002300 \\
0.00005900 \\
-0.00000900 \\
-0.00004900 \\
-0.88580300 \\
-0.00000700 \\
-0.00003200 \\
-0.00001700 \\
-0.00000600\end{array}$ \\
\hline & \multicolumn{4}{|c|}{$\begin{array}{l}\text { Free Energy: }-504.366875 \\
\text { Enthalpy: }-504.315255 \\
\text { ZPE Corrected Energy: }-504.327998\end{array}$} \\
\hline
\end{tabular}




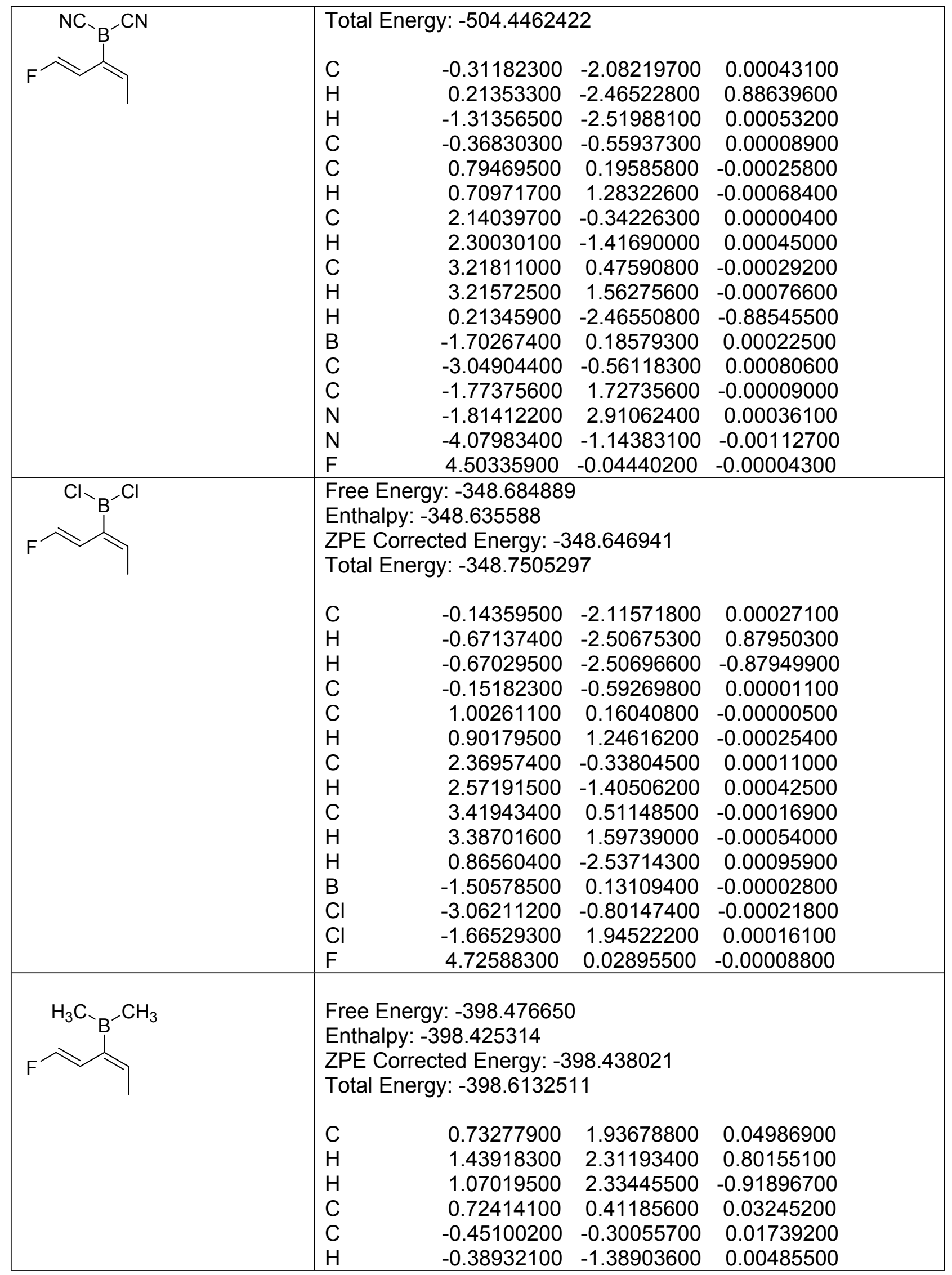




\begin{tabular}{|c|c|c|c|c|}
\hline & $\begin{array}{l}\mathrm{C} \\
\mathrm{H} \\
\mathrm{C} \\
\mathrm{H} \\
\mathrm{H} \\
\mathrm{B} \\
\mathrm{C} \\
\mathrm{H} \\
\mathrm{H} \\
\mathrm{H} \\
\mathrm{C} \\
\mathrm{H} \\
\mathrm{H} \\
\mathrm{H} \\
\mathrm{F}\end{array}$ & $\begin{array}{c}-1.81022000 \\
-1.97601400 \\
-2.89078200 \\
-2.90397900 \\
-0.24356800 \\
2.10115800 \\
2.22928200 \\
2.96574500 \\
1.30882300 \\
2.65337000 \\
3.44733900 \\
3.60964900 \\
4.33191500 \\
3.40267800 \\
-4.18485300\end{array}$ & $\begin{array}{r}0.23553500 \\
1.30958900 \\
-0.57073100 \\
-1.65673800 \\
2.38385900 \\
-0.35071800 \\
-1.93115800 \\
-2.21990600 \\
-2.49646800 \\
-2.27730800 \\
0.48909400 \\
1.00174600 \\
-0.12918500 \\
1.28726300 \\
-0.03684300\end{array}$ & $\begin{array}{r}0.00516900 \\
0.00777400 \\
-0.01618100 \\
-0.02596400 \\
0.26570500 \\
-0.00460000 \\
0.03899800 \\
0.80532300 \\
0.22212900 \\
-0.91886900 \\
-0.08127900 \\
0.88334400 \\
-0.27691200 \\
-0.83685900 \\
-0.02984800\end{array}$ \\
\hline 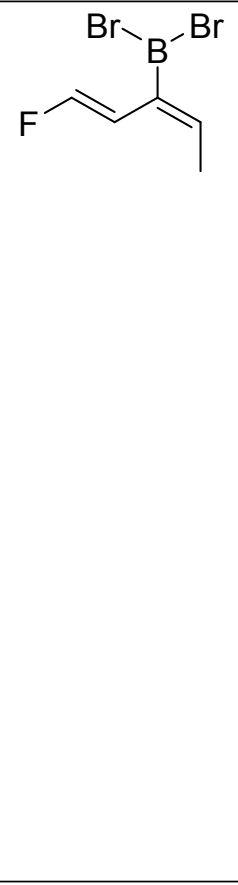 & $\begin{array}{l}\text { Fre } \\
\text { En } \\
\text { ZP } \\
\text { To } \\
\text { C } \\
\mathrm{H} \\
\mathrm{H} \\
\mathrm{C} \\
\mathrm{C} \\
\mathrm{H} \\
\mathrm{C} \\
\mathrm{H} \\
\mathrm{C} \\
\mathrm{H} \\
\mathrm{H} \\
\mathrm{B} \\
\mathrm{Br} \\
\mathrm{Br} \\
\mathrm{F}\end{array}$ & $\begin{array}{r}0.60240700 \\
0.07529700 \\
0.07463600 \\
0.59875200 \\
1.76467600 \\
1.68043100 \\
3.12545200 \\
3.31725100 \\
4.18490400 \\
4.16499400 \\
1.61064000 \\
-0.75386700 \\
-0.92028500 \\
-2.45637100 \\
5.48576900\end{array}$ & $\begin{array}{l} \\
45.070011 \\
-2.31288600 \\
-2.70317800 \\
-2.70302200 \\
-0.78850100 \\
-0.05090100 \\
1.03609100 \\
-0.56534300 \\
-1.63424200 \\
0.27257000 \\
1.35875700 \\
-2.73642900 \\
-0.05191500 \\
1.92982900 \\
-1.06306200 \\
-0.22498500\end{array}$ & $\begin{array}{r}-0.00039000 \\
0.87931900 \\
-0.87976700 \\
-0.00029700 \\
-0.00030500 \\
-0.00020200 \\
-0.00024500 \\
-0.00020200 \\
0.00006300 \\
0.00026200 \\
-0.00081500 \\
-0.00010700 \\
-0.00005400 \\
0.00018900 \\
0.00047500 \\
\end{array}$ \\
\hline$\underbrace{\mathrm{H}_{3} \mathrm{C} \mathrm{B}^{-} \mathrm{CH}_{3}}_{F}$ & $\begin{array}{l}\text { Fre } \\
\text { En } \\
\text { ZP } \\
\text { To } \\
\text { C } \\
\text { H } \\
\text { C } \\
\text { C } \\
\text { H } \\
\text { C } \\
\text { H } \\
\text { C } \\
\text { H } \\
\text { H }\end{array}$ & $\begin{array}{l}y:-398.475179 \\
398.424495 \\
\text { ted Energy: }-3 \\
\text { y: } 398.613002 \\
0.21990900 \\
0.81885000 \\
0.07004100 \\
-1.17123000 \\
-1.21498800 \\
-2.48501400 \\
-2.53431400 \\
-3.64802400 \\
-3.65751300 \\
-0.71717500\end{array}$ & $\begin{array}{l} \\
98.436954 \\
28 \\
-1.59042700 \\
-1.83655300 \\
-0.10034300 \\
0.48080000 \\
1.56360500 \\
-0.16211500 \\
-1.24567200 \\
0.53640000 \\
1.62172000 \\
-2.15269200 \\
\end{array}$ & $\begin{array}{r}0.31097000 \\
1.19498300 \\
0.12219000 \\
0.03964500 \\
-0.08730500 \\
0.12281200 \\
0.21556400 \\
0.07369300 \\
-0.01715000 \\
0.32771200 \\
\end{array}$ \\
\hline
\end{tabular}




\begin{tabular}{|c|c|c|c|c|}
\hline & $\begin{array}{l}\mathrm{B} \\
\mathrm{C} \\
\mathrm{H} \\
\mathrm{H} \\
\mathrm{H} \\
\mathrm{C} \\
\mathrm{H} \\
\mathrm{H} \\
\mathrm{H} \\
\mathrm{H} \\
\mathrm{F}\end{array}$ & $\begin{array}{r}1.37792300 \\
1.38755300 \\
2.13398600 \\
0.43757000 \\
1.74508000 \\
2.73721600 \\
3.02017200 \\
3.56770100 \\
2.62898600 \\
-4.61482100 \\
0.98157300\end{array}$ & $\begin{array}{r}0.77655400 \\
2.25608800 \\
2.31606000 \\
2.63378200 \\
2.94950700 \\
0.16693400 \\
-0.70404400 \\
0.88256700 \\
-0.21290300 \\
0.04177300 \\
-2.14043800\end{array}$ & $\begin{array}{r}0.08172000 \\
-0.48142100 \\
-1.29036200 \\
-0.87586300 \\
0.29705400 \\
0.62494700 \\
0.01307000 \\
0.61253600 \\
1.65390200 \\
0.12210700 \\
-0.82798500\end{array}$ \\
\hline$\overbrace{F}^{\mathrm{Cl}_{\mathrm{B}^{-}}-\mathrm{Cl}}$ & $\begin{array}{l}\text { Fre } \\
\text { En } \\
\text { ZP } \\
\text { To } \\
\text { C } \\
\mathrm{H} \\
\mathrm{C} \\
\mathrm{C} \\
\mathrm{H} \\
\mathrm{C} \\
\mathrm{H} \\
\mathrm{C} \\
\mathrm{H} \\
\mathrm{H} \\
\mathrm{B} \\
\mathrm{H} \\
\mathrm{F} \\
\mathrm{Cl} \\
\mathrm{Cl}\end{array}$ & $\begin{array}{l}:-348.681212 \\
48.632662 \\
\text { ted Energy: }-3 \\
:-348.748177 \\
0.55463000 \\
-0.34366000 \\
0.26231300 \\
1.30505300 \\
1.06535800 \\
2.72719600 \\
3.02740400 \\
3.67364800 \\
3.40820200 \\
1.07884300 \\
-1.18218200 \\
4.73347000 \\
1.45377800 \\
-2.58817900 \\
-1.60474700\end{array}$ & $\begin{array}{l} \\
48.643709 \\
2 \\
-1.74652100 \\
-2.36321500 \\
-0.27323200 \\
0.62770800 \\
1.68219900 \\
0.31793200 \\
-0.72314600 \\
1.29299200 \\
2.34436100 \\
-1.93221200 \\
0.21844800 \\
1.06103900 \\
-2.24934800 \\
-0.92826300 \\
1.97353300\end{array}$ & $\begin{array}{r}-0.35979200 \\
-0.30363000 \\
-0.14681800 \\
-0.06646000 \\
0.07531200 \\
-0.15002600 \\
-0.22810800 \\
-0.11497600 \\
-0.01160800 \\
-1.30568000 \\
-0.03381100 \\
-0.18388000 \\
0.68836400 \\
-0.12539500 \\
0.18185400\end{array}$ \\
\hline$\overbrace{F}^{N C^{-}{ }^{-C N}}$ & $\begin{array}{l}\mathrm{C} \\
\mathrm{H} \\
\mathrm{C} \\
\mathrm{C} \\
\mathrm{H} \\
\mathrm{C} \\
\mathrm{H} \\
\mathrm{C} \\
\mathrm{H} \\
\mathrm{H} \\
\mathrm{B} \\
\mathrm{H} \\
\mathrm{F} \\
\mathrm{C}\end{array}$ & $\begin{array}{r}0.27748000 \\
-0.64492600 \\
0.07687200 \\
1.16373300 \\
0.96295300 \\
2.56899000 \\
2.83201500 \\
3.54698100 \\
3.31442000 \\
0.70518000 \\
-1.33058600 \\
4.59897600 \\
1.23456000 \\
-2.58071000 \\
\end{array}$ & $\begin{array}{l}\text { D4.324665 } \\
-1.75292000 \\
-2.32014500 \\
-0.26474100 \\
0.59464600 \\
1.65970000 \\
0.23178000 \\
-0.81924400 \\
1.17850000 \\
2.23835200 \\
-1.98239900 \\
0.31195000 \\
0.91243500 \\
-2.28565000 \\
-0.58657300 \\
\end{array}$ & $\begin{array}{r}-0.34173300 \\
-0.19778200 \\
-0.13589000 \\
-0.06182900 \\
0.06793300 \\
-0.13657700 \\
-0.20835200 \\
-0.10351200 \\
-0.00736600 \\
-1.32631600 \\
-0.03035500 \\
-0.16846100 \\
0.63400700 \\
-0.07684300\end{array}$ \\
\hline
\end{tabular}




\begin{tabular}{|c|c|c|c|c|}
\hline & $\begin{array}{l}\mathrm{C} \\
\mathrm{N} \\
\mathrm{N}\end{array}$ & $\begin{array}{l}-1.57838000 \\
-3.53740200 \\
-1.75924400 \\
\end{array}$ & $\begin{array}{r}1.82658600 \\
-1.28293600 \\
2.99132800 \\
\end{array}$ & $\begin{array}{r}0.11365100 \\
-0.11642700 \\
0.22249200 \\
\end{array}$ \\
\hline $\mathrm{F}_{\backslash_{B^{-}}}-\mathrm{F}$ & $\begin{array}{l}\mathrm{C} \\
\mathrm{H} \\
\mathrm{C} \\
\mathrm{C} \\
\mathrm{H} \\
\mathrm{C} \\
\mathrm{H} \\
\mathrm{C} \\
\mathrm{H} \\
\mathrm{H} \\
\mathrm{B} \\
\mathrm{H} \\
\mathrm{F} \\
\mathrm{F} \\
\mathrm{F}\end{array}$ & $\begin{array}{l}\text { : }-518.532815 \\
18.486758 \\
\text { ted Energy: }-51 \\
y:-518.604570 \varepsilon \\
-0.17680700 \\
-1.13583900 \\
-0.29241200 \\
0.80903800 \\
0.64103400 \\
2.20678400 \\
2.42192200 \\
3.22449700 \\
3.04000500 \\
0.24252100 \\
-1.68172600 \\
4.26377600 \\
0.74086100 \\
-1.88219500 \\
-2.82437600\end{array}$ & $\begin{array}{l}\text { 18.497153 } \\
8 \\
1.59159000 \\
2.10390200 \\
0.10872900 \\
-0.70546900 \\
-1.76867200 \\
-0.29472800 \\
0.76415200 \\
-1.19152600 \\
-2.25628700 \\
1.80021200 \\
-0.50607800 \\
-0.88154300 \\
2.23375200 \\
-1.84801800 \\
0.24949300 \\
\end{array}$ & $\begin{array}{r}0.40878400 \\
0.29570800 \\
0.13429600 \\
0.01873100 \\
-0.16275200 \\
0.10816400 \\
0.22223800 \\
0.03721900 \\
-0.10063700 \\
1.40145500 \\
-0.00337800 \\
0.11168100 \\
-0.54101800 \\
-0.20990900 \\
0.08493100\end{array}$ \\
\hline & $\begin{array}{l}\text { Fre } \\
\text { Ent } \\
\text { ZPE } \\
\text { Tot } \\
\text { C } \\
\text { H } \\
\text { C } \\
\text { C } \\
\text { H } \\
\text { C } \\
\text { H } \\
\text { C } \\
\text { H } \\
\text { H } \\
\text { B } \\
\text { H } \\
\text { H } \\
\text { H } \\
\text { F }\end{array}$ & $\begin{array}{l}\text { : }-319.874869 \\
19.833283 \\
\text { ted Energy: }-31 \\
\text { y: }-319.960785 \\
\\
1.44858000 \\
2.51418300 \\
0.87022500 \\
-0.49210700 \\
-0.82602500 \\
-1.55679400 \\
-1.27255000 \\
-2.87001600 \\
-3.18373600 \\
1.29208200 \\
1.81010000 \\
-3.65956600 \\
2.99931000 \\
1.35253000 \\
0.81488300\end{array}$ & $\begin{array}{l}1 \\
19.842244 \\
2 \\
-0.76439200 \\
-0.81161000 \\
0.59846100 \\
0.80291700 \\
1.82129800 \\
-0.18824000 \\
-1.22536100 \\
0.15495400 \\
1.18390000 \\
-1.04625700 \\
1.80093800 \\
-0.58405000 \\
1.67844900 \\
2.88869700 \\
-1.83688400 \\
\end{array}$ & $\begin{array}{r}0.38337900 \\
0.14217000 \\
0.06114200 \\
-0.04565000 \\
-0.25608600 \\
0.08985200 \\
0.24167900 \\
0.02120500 \\
-0.15191500 \\
1.43380600 \\
-0.12239900 \\
0.13352400 \\
-0.07378000 \\
-0.31587000 \\
-0.40012200\end{array}$ \\
\hline$\overbrace{F}^{\mathrm{H}_{2} \mathrm{~N}_{-}{ }^{-} \mathrm{NH}_{2}}$ & \multicolumn{4}{|c|}{$\begin{array}{l}\text { Free Energy: }-430.663498 \\
\text { Enthalpy: }-430.616716 \\
\text { ZPE Corrected Energy: }-430.627882 \\
\text { Total Energy: }-430.7857902\end{array}$} \\
\hline
\end{tabular}




\begin{tabular}{|c|c|c|c|c|}
\hline & $\begin{array}{l}\mathrm{C} \\
\mathrm{H} \\
\mathrm{C} \\
\mathrm{C} \\
\mathrm{H} \\
\mathrm{C} \\
\mathrm{H} \\
\mathrm{C} \\
\mathrm{H} \\
\mathrm{H} \\
\mathrm{B} \\
\mathrm{H} \\
\mathrm{N} \\
\mathrm{H} \\
\mathrm{H} \\
\mathrm{N} \\
\mathrm{H} \\
\mathrm{H} \\
\mathrm{F}\end{array}$ & $\begin{array}{r}0.25357600 \\
0.84436200 \\
0.09238700 \\
-1.14476600 \\
-1.19403200 \\
-2.45243700 \\
-2.48850500 \\
-3.62310300 \\
-3.65014300 \\
-0.67914600 \\
1.40748800 \\
-4.57916400 \\
1.35199800 \\
0.53067400 \\
2.13733500 \\
2.61796600 \\
3.51009100 \\
2.61457800 \\
1.04142400 \\
\end{array}$ & $\begin{array}{r}-1.46673300 \\
-1.53504500 \\
-0.04247200 \\
0.48157700 \\
1.52039800 \\
-0.17608800 \\
-1.22719300 \\
0.45880400 \\
1.50820500 \\
-2.02505800 \\
0.82757500 \\
-0.05415500 \\
2.23890800 \\
2.69294700 \\
2.85833000 \\
0.17187600 \\
0.64073900 \\
-0.81061900 \\
-2.23471500 \\
\end{array}$ & $\begin{array}{r}0.64456900 \\
1.56505300 \\
0.17572800 \\
-0.08367000 \\
-0.41839000 \\
0.01002300 \\
0.29317000 \\
-0.25061500 \\
-0.54116800 \\
0.75166400 \\
0.00425800 \\
-0.18382300 \\
0.23010900 \\
0.60575300 \\
0.07401200 \\
-0.35767900 \\
-0.44839200 \\
-0.60403000 \\
-0.35537400 \\
\end{array}$ \\
\hline & $\begin{array}{l}\mathrm{C} \\
\mathrm{H} \\
\mathrm{C} \\
\mathrm{C} \\
\mathrm{H} \\
\mathrm{C} \\
\mathrm{H} \\
\mathrm{C} \\
\mathrm{H} \\
\mathrm{H} \\
\mathrm{B} \\
\mathrm{H} \\
\mathrm{F} \\
\mathrm{O} \\
\mathrm{H} \\
\mathrm{O} \\
\mathrm{H}\end{array}$ & $\begin{array}{r}-0.14571200 \\
-1.10504900 \\
-0.27235800 \\
0.82096500 \\
0.64030900 \\
2.22442700 \\
2.44062000 \\
3.24639000 \\
3.06550700 \\
0.28601400 \\
-1.69638400 \\
4.28455900 \\
0.76847800 \\
-1.83562300 \\
-2.69589900 \\
-2.77563500 \\
-3.70265600 \\
\end{array}$ & $\begin{array}{l}70.400000 \\
6 \\
1.58473800 \\
2.09389900 \\
0.10271700 \\
-0.71423700 \\
-1.77521900 \\
-0.30994600 \\
0.74997500 \\
-1.20141000 \\
-2.26746600 \\
1.79786100 \\
-0.49555900 \\
-0.88701800 \\
2.23140000 \\
-1.86174500 \\
-2.30381100 \\
0.37682000 \\
0.09519200 \\
\end{array}$ & $\begin{array}{r}0.40264600 \\
0.29247300 \\
0.13293500 \\
0.01971500 \\
-0.15836100 \\
0.10995500 \\
0.21999200 \\
0.04621400 \\
-0.08723800 \\
1.38967300 \\
0.00242700 \\
0.12113600 \\
-0.55748500 \\
-0.21438900 \\
-0.30937800 \\
0.11834800 \\
0.03646900 \\
\end{array}$ \\
\hline$\overbrace{F}^{-B-B r}$ & \multicolumn{4}{|c|}{$\begin{array}{l}\text { Free Energy: }-345.055474 \\
\text { Enthalpy: -345.007086 } \\
\text { ZPE Corrected Energy: }-345.017478 \\
\text { Total Energy: }-345.116279 \\
\text { Frequency: }-1354.9440\end{array}$} \\
\hline
\end{tabular}




\begin{tabular}{|c|c|c|c|c|}
\hline & $\begin{array}{l}\mathrm{H} \\
\mathrm{C} \\
\mathrm{C} \\
\mathrm{H} \\
\mathrm{C} \\
\mathrm{H} \\
\mathrm{C} \\
\mathrm{H} \\
\mathrm{H} \\
\mathrm{B} \\
\mathrm{Br} \\
\mathrm{Br} \\
\mathrm{F}\end{array}$ & $\begin{array}{c}-1.02598800 \\
-0.81954400 \\
-1.61580700 \\
-1.12991000 \\
-2.98499500 \\
-3.47527700 \\
-3.72466600 \\
-4.08598300 \\
-2.68054800 \\
0.67150700 \\
1.72175700 \\
1.65801000 \\
-4.79921100\end{array}$ & $\begin{array}{r}2.14903000 \\
-0.00719000 \\
-1.16374200 \\
-2.10863400 \\
-1.05257400 \\
-1.83518500 \\
0.16394300 \\
0.49259100 \\
1.08261800 \\
0.01113500 \\
-1.66374700 \\
1.70444600 \\
0.31664500\end{array}$ & $\begin{array}{r}0.75400400 \\
0.47836200 \\
0.21561400 \\
-0.01056700 \\
-0.04362000 \\
-0.62001400 \\
0.14862800 \\
1.12519600 \\
0.13813100 \\
0.12227300 \\
-0.02779400 \\
-0.17083400 \\
-0.77902500\end{array}$ \\
\hline $\mathrm{H}_{3} \mathrm{C}_{-}-\mathrm{CH}_{3}$ & $\begin{array}{l}\mathrm{C} \\
\mathrm{H} \\
\mathrm{H} \\
\mathrm{C} \\
\mathrm{C} \\
\mathrm{H} \\
\mathrm{C} \\
\mathrm{H} \\
\mathrm{C} \\
\mathrm{H} \\
\mathrm{H} \\
\mathrm{B} \\
\mathrm{F} \\
\mathrm{C} \\
\mathrm{H} \\
\mathrm{H} \\
\mathrm{H} \\
\mathrm{C} \\
\mathrm{H} \\
\mathrm{H} \\
\mathrm{H}\end{array}$ & $\begin{array}{r}0.14681600 \\
0.66305400 \\
-0.35810900 \\
-0.52569700 \\
0.27581200 \\
-0.20549500 \\
1.66052500 \\
2.16351100 \\
2.39916300 \\
2.69551500 \\
1.35137700 \\
-2.02458000 \\
3.55174000 \\
-2.87977500 \\
-2.46361000 \\
-2.95784500 \\
-3.91109600 \\
-2.74031800 \\
-2.06628800 \\
-3.24239300 \\
-3.53054000\end{array}$ & $\begin{array}{l} \\
98.388224 \\
23 \\
-1.25948300 \\
-1.28522500 \\
-2.20397900 \\
-0.03878500 \\
1.12429500 \\
2.07614300 \\
1.03530700 \\
1.83746700 \\
-0.17844500 \\
-0.55478100 \\
-1.10993500 \\
-0.02638800 \\
-0.26577300 \\
1.30886000 \\
2.08919500 \\
1.72918800 \\
1.10129100 \\
-1.34528100 \\
-2.09237300 \\
-1.82660800 \\
-1.11529000\end{array}$ & $\begin{array}{r}0.74014600 \\
1.70576100 \\
0.52726100 \\
0.37417700 \\
0.21167900 \\
-0.00122900 \\
-0.00156500 \\
-0.53981900 \\
0.17961600 \\
1.16061100 \\
0.06175600 \\
-0.06966100 \\
-0.67218800 \\
0.00956400 \\
0.65790500 \\
-1.00874100 \\
0.32824900 \\
-0.58536100 \\
-1.02306600 \\
0.27250800 \\
-1.31272800\end{array}$ \\
\hline $\mathrm{Cl}_{\mathrm{B}}$ & \multicolumn{4}{|c|}{$\begin{array}{l}\text { Free Energy: }-348.629712 \\
\text { Enthalpy: }-348.584052 \\
\text { ZPE Corrected Energy: }-348.593966 \\
\text { Total Energy: }-348.6939729 \\
\text { Frequency: }-1363.0999\end{array}$} \\
\hline
\end{tabular}




\begin{tabular}{|c|c|c|c|c|}
\hline & $\begin{array}{l}\mathrm{H} \\
\mathrm{C} \\
\mathrm{H} \\
\mathrm{C} \\
\mathrm{H} \\
\mathrm{H} \\
\mathrm{B} \\
\mathrm{Cl} \\
\mathrm{Cl} \\
\mathrm{F}\end{array}$ & $\begin{array}{r}0.37621700 \\
2.23030900 \\
2.73732900 \\
2.95792000 \\
3.29116800 \\
1.91059100 \\
-1.42738700 \\
-2.35435200 \\
-2.35801900 \\
4.05473900\end{array}$ & $\begin{array}{r}2.09014900 \\
1.03269100 \\
1.86451300 \\
-0.20261300 \\
-0.63184300 \\
-1.10919400 \\
-0.01632900 \\
1.54184500 \\
-1.53659900 \\
-0.27068600\end{array}$ & $\begin{array}{r}0.15385600 \\
0.06222000 \\
-0.42351900 \\
0.14917000 \\
1.09626900 \\
0.01819400 \\
0.03887100 \\
-0.06882800 \\
-0.30447300 \\
-0.76178900\end{array}$ \\
\hline NC: & $\begin{array}{l}\mathrm{C} \\
\mathrm{H} \\
\mathrm{H} \\
\mathrm{C} \\
\mathrm{C} \\
\mathrm{H} \\
\mathrm{C} \\
\mathrm{H} \\
\mathrm{C} \\
\mathrm{H} \\
\mathrm{H} \\
\mathrm{B} \\
\mathrm{C} \\
\mathrm{C} \\
\mathrm{N} \\
\mathrm{N} \\
\mathrm{F}\end{array}$ & $\begin{array}{l}y:-504.31193 \\
04.263666 \\
\text { ted Energy: }-5 \\
y:-504.38952 \\
-1309.0078 \\
\\
0.54049300 \\
1.05809000 \\
0.05401600 \\
-0.13537900 \\
0.66703700 \\
0.18315800 \\
2.03862100 \\
2.55398400 \\
2.76405700 \\
3.10363200 \\
1.72929200 \\
-1.61157300 \\
-2.39982100 \\
-2.38562600 \\
-2.99549700 \\
-2.96696300 \\
3.84184800\end{array}$ & $\begin{array}{l} \\
04.275041 \\
95 \\
-1.28351100 \\
-1.35004100 \\
-2.22105600 \\
-0.04522400 \\
1.13426200 \\
2.09770500 \\
1.04307100 \\
1.87453400 \\
-0.19737700 \\
-0.61049400 \\
-1.10578700 \\
-0.01822000 \\
-1.31476900 \\
1.30999100 \\
-2.31704200 \\
2.33816500 \\
-0.29114200\end{array}$ & $\begin{array}{r}0.71475400 \\
1.67631300 \\
0.44020100 \\
0.40549200 \\
0.28577200 \\
0.14745300 \\
0.05383700 \\
-0.42310100 \\
0.15086300 \\
1.10321800 \\
0.02393200 \\
0.03271000 \\
-0.22925800 \\
-0.06757900 \\
-0.43455100 \\
-0.14712800 \\
-0.77145500\end{array}$ \\
\hline$\overbrace{F}^{B_{1}}$ & $\begin{array}{l}\mathrm{C} \\
\mathrm{H} \\
\mathrm{H} \\
\mathrm{C} \\
\mathrm{C} \\
\mathrm{H} \\
\mathrm{C} \\
\mathrm{H} \\
\mathrm{C} \\
\mathrm{H} \\
\mathrm{H} \\
\mathrm{B}\end{array}$ & $\begin{array}{l}y:-518.48025 \\
18.437112 \\
\text { ted Energy: }-5 \\
y:-518.54941 \\
-1388.8841 \\
0.07450600 \\
0.59036800 \\
-0.44281300 \\
-0.56929200 \\
0.23128500 \\
-0.24619300 \\
1.61655800 \\
2.14165700 \\
2.33465200 \\
2.61551700 \\
1.28847600 \\
-2.03054000\end{array}$ & $\begin{array}{l} \\
18.446363 \\
25 \\
-1.36147400 \\
-1.54798600 \\
-2.24864700 \\
-0.08775200 \\
1.08622000 \\
2.06067200 \\
1.01287600 \\
1.88909500 \\
-0.22829300 \\
-0.77352500 \\
-1.11124900 \\
0.00587100\end{array}$ & $\begin{array}{r}0.50676600 \\
1.45320600 \\
0.13820100 \\
0.33776300 \\
0.37439200 \\
0.31287800 \\
0.18208100 \\
-0.19407200 \\
0.15632900 \\
1.05967000 \\
-0.13341300 \\
-0.09489000\end{array}$ \\
\hline
\end{tabular}




\begin{tabular}{|c|c|c|c|c|}
\hline & $\begin{array}{l}F \\
F \\
F\end{array}$ & $\begin{array}{r}-2.70314000 \\
-2.76797100 \\
3.47993600\end{array}$ & $\begin{array}{r}1.20080100 \\
-1.09849700 \\
-0.19421200\end{array}$ & $\begin{array}{l}-0.14629500 \\
-0.43541200 \\
-0.69673700\end{array}$ \\
\hline 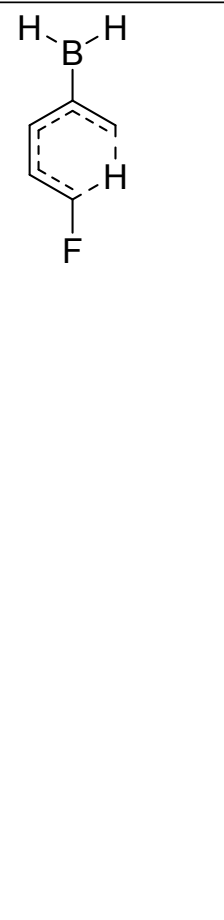 & $\begin{array}{l}\mathrm{C} \\
\mathrm{H} \\
\mathrm{H} \\
\mathrm{C} \\
\mathrm{C} \\
\mathrm{H} \\
\mathrm{C} \\
\mathrm{H} \\
\mathrm{C} \\
\mathrm{H} \\
\mathrm{H} \\
\mathrm{B} \\
\mathrm{F} \\
\mathrm{H} \\
\mathrm{H}\end{array}$ & $\begin{array}{r}0.83429600 \\
0.45011800 \\
1.31701700 \\
1.44130200 \\
0.61738900 \\
1.07238200 \\
-0.77897000 \\
-1.35544300 \\
-1.47228100 \\
-1.65112300 \\
-0.44158100 \\
2.84939300 \\
-2.70085100 \\
3.36118100 \\
3.45772100\end{array}$ & $\begin{array}{l} \\
19.792904 \\
11 \\
1.34626600 \\
1.60798700 \\
2.19396100 \\
0.04734300 \\
-1.10143000 \\
-2.08857600 \\
-1.01015500 \\
-1.88747100 \\
0.24560600 \\
0.82523000 \\
1.09538600 \\
-0.09966500 \\
0.21093900 \\
0.80895400 \\
-1.12138000\end{array}$ & $\begin{array}{r}0.26801300 \\
1.25968100 \\
-0.22367300 \\
0.09828400 \\
0.28481700 \\
0.23611200 \\
0.21272800 \\
-0.07607800 \\
0.21336800 \\
1.12106800 \\
-0.21320300 \\
-0.49187700 \\
-0.51930000 \\
-1.07751200 \\
-0.35656900\end{array}$ \\
\hline (- & $\begin{array}{l}\mathrm{C} \\
\mathrm{H} \\
\mathrm{H} \\
\mathrm{C} \\
\mathrm{C} \\
\mathrm{H} \\
\mathrm{C} \\
\mathrm{H} \\
\mathrm{C} \\
\mathrm{H} \\
\mathrm{H} \\
\mathrm{B} \\
\mathrm{N} \\
\mathrm{H} \\
\mathrm{H} \\
\mathrm{N} \\
\mathrm{H} \\
\mathrm{H} \\
\mathrm{F}\end{array}$ & $\begin{array}{r}0.11272500 \\
0.64907900 \\
-0.41077100 \\
-0.56027100 \\
0.23231100 \\
-0.24997600 \\
1.62407200 \\
2.12241900 \\
2.37289300 \\
2.65670800 \\
1.32530200 \\
-2.07304700 \\
-2.59615700 \\
-3.56412900 \\
-2.01015300 \\
-2.88968500 \\
-2.54931700 \\
-3.85827100 \\
3.55165100\end{array}$ & $\begin{array}{l}\text { 30.574501 } \\
76 \\
\\
-1.16501300 \\
-1.06122000 \\
-2.12294100 \\
-0.00555900 \\
1.12875900 \\
2.03214300 \\
1.02627300 \\
1.76173700 \\
-0.14898300 \\
-0.41385900 \\
-1.09907000 \\
-0.05057900 \\
-1.22659000 \\
-1.32973300 \\
-2.01561700 \\
1.09954300 \\
1.90251400 \\
1.16623000 \\
-0.29898000\end{array}$ & $\begin{array}{r}0.88244000 \\
1.83136300 \\
0.83153600 \\
0.36733200 \\
0.05916900 \\
-0.30924400 \\
-0.11824100 \\
-0.74884800 \\
0.20545000 \\
1.22599900 \\
0.18505500 \\
-0.09126900 \\
-0.70847000 \\
-0.98580900 \\
-0.94570400 \\
0.13520800 \\
0.64698900 \\
-0.15139100 \\
-0.60974000\end{array}$ \\
\hline
\end{tabular}




\begin{tabular}{|c|c|c|c|c|}
\hline$\underbrace{-\mathrm{HO}}_{\mathrm{F}}$ & $\begin{array}{l}\mathrm{C} \\
\mathrm{H} \\
\mathrm{H} \\
\mathrm{C} \\
\mathrm{C} \\
\mathrm{H} \\
\mathrm{C} \\
\mathrm{H} \\
\mathrm{C} \\
\mathrm{H} \\
\mathrm{H} \\
\mathrm{B} \\
\mathrm{O} \\
\mathrm{H} \\
\mathrm{O} \\
\mathrm{H} \\
\mathrm{F}\end{array}$ & $\begin{array}{l}:-470.38625 \\
70.342378 \\
\text { ted Energy: }-4 \\
y:-470.47933 \\
-1431.1918 \\
\\
-0.09602400 \\
-0.62185300 \\
0.40968300 \\
0.56156100 \\
-0.23796700 \\
0.25414800 \\
-1.62549000 \\
-2.13403500 \\
-2.35945600 \\
-2.63900000 \\
-1.31176400 \\
2.05380200 \\
2.87271000 \\
2.53319100 \\
2.62028000 \\
3.56297600 \\
-3.52466900\end{array}$ & $\begin{array}{l} \\
70.352107 \\
13 \\
\\
1.38725100 \\
1.60271100 \\
2.28198900 \\
0.11574500 \\
-1.05384000 \\
-2.02116900 \\
-0.99804400 \\
-1.88830000 \\
0.22987900 \\
0.79631900 \\
1.11329800 \\
-0.02136500 \\
1.05264100 \\
1.96249600 \\
-1.28085700 \\
-1.34935400 \\
0.14984600\end{array}$ & $\begin{array}{r}0.46678100 \\
1.40201200 \\
0.08909400 \\
0.34087800 \\
0.40103700 \\
0.35690200 \\
0.19625900 \\
-0.17112600 \\
0.14090800 \\
1.03188700 \\
-0.17091000 \\
-0.08752500 \\
-0.41559600 \\
-0.37256200 \\
-0.13333600 \\
-0.37163000 \\
-0.69330700\end{array}$ \\
\hline $\mathrm{H}$ & $\begin{array}{l}\mathrm{C} \\
\mathrm{H} \\
\mathrm{H} \\
\mathrm{C} \\
\mathrm{C} \\
\mathrm{H} \\
\mathrm{C} \\
\mathrm{H} \\
\mathrm{C} \\
\mathrm{H} \\
\mathrm{H} \\
\mathrm{H} \\
\mathrm{F}\end{array}$ & $\begin{array}{l}:-294.36243 \\
\text { ted Energy: }-2 \\
y:-294.43836 \\
-682.4800 \\
-1.45085700 \\
-0.96078600 \\
-2.35447800 \\
-1.26008100 \\
0.00021900 \\
0.00050100 \\
1.26026400 \\
2.08214600 \\
1.45112100 \\
0.96113800 \\
2.35453500 \\
-2.08147900 \\
-0.00062000 \\
\end{array}$ & $\begin{array}{l} \\
94.333324 \\
87 \\
-0.48509300 \\
-0.92340400 \\
-1.00551300 \\
0.84342500 \\
1.48078100 \\
2.50128400 \\
0.84282300 \\
1.33989700 \\
-0.48572400 \\
-0.92462700 \\
-1.00614200 \\
1.33997300 \\
-1.61097200 \\
\end{array}$ & $\begin{array}{r}0.39403400 \\
1.24964100 \\
0.07951400 \\
-0.01371100 \\
-0.08915400 \\
-0.47581100 \\
-0.01371900 \\
-0.52758400 \\
0.39367000 \\
1.24902000 \\
0.07853000 \\
-0.52883300 \\
-0.57235600 \\
\end{array}$ \\
\hline$\overbrace{H}^{-B r}$ & \multicolumn{4}{|c|}{$\begin{array}{l}\text { Free Energy: }-345.032308 \\
\text { Enthalpy: }-344.984181 \\
\text { ZPE Corrected Energy: }-344.994734 \\
\text { Total Energy: }-345.096581 \\
\text { Frequency: }-743.0930\end{array}$} \\
\hline
\end{tabular}




\begin{tabular}{|c|c|c|c|c|}
\hline & $\begin{array}{l}\mathrm{C} \\
\mathrm{C} \\
\mathrm{H} \\
\mathrm{C} \\
\mathrm{H} \\
\mathrm{C} \\
\mathrm{H} \\
\mathrm{B} \\
\mathrm{H} \\
\mathrm{Br} \\
\mathrm{Br} \\
\mathrm{F}\end{array}$ & $\begin{array}{c}-0.95997600 \\
-1.70742400 \\
-1.12656100 \\
-3.07818500 \\
-3.42973000 \\
-3.98988600 \\
-4.12830400 \\
0.53075200 \\
-4.87635600 \\
1.63017600 \\
1.53848700 \\
-3.09676700\end{array}$ & $\begin{array}{r}-0.06113800 \\
-1.27921300 \\
-2.18085000 \\
-1.36530900 \\
-2.24608400 \\
-0.27398200 \\
0.26563200 \\
-0.01591200 \\
-0.27935800 \\
-1.67330900 \\
1.70133300 \\
1.24444700\end{array}$ & $\begin{array}{r}0.35022200 \\
0.16934100 \\
-0.01851000 \\
-0.08079500 \\
-0.61546600 \\
0.04065200 \\
0.97136600 \\
0.10127100 \\
-0.59465900 \\
-0.03714900 \\
-0.05233800 \\
-0.60816300\end{array}$ \\
\hline $\mathrm{H}_{3} \mathrm{C}_{-}-\mathrm{CH}_{3}$ & $\begin{array}{l}\mathrm{C} \\
\mathrm{H} \\
\mathrm{H} \\
\mathrm{C} \\
\mathrm{C} \\
\mathrm{H} \\
\mathrm{C} \\
\mathrm{H} \\
\mathrm{C} \\
\mathrm{H} \\
\mathrm{B} \\
\mathrm{H} \\
\mathrm{F} \\
\mathrm{C} \\
\mathrm{H} \\
\mathrm{H} \\
\mathrm{H} \\
\mathrm{C} \\
\mathrm{H} \\
\mathrm{H} \\
\mathrm{H}\end{array}$ & $\begin{array}{l}y:-398.397953 \\
398.350670 \\
\text { ted Energy: }-3 \\
y:-398.536655 \\
-705.4898 \\
0.39473900 \\
1.13904000 \\
-0.08122900 \\
-0.29357600 \\
0.44984600 \\
-0.13353900 \\
1.82993200 \\
2.15912700 \\
2.75197400 \\
2.81604900 \\
-1.81034400 \\
3.68042200 \\
1.91374800 \\
-2.68078700 \\
-2.25005400 \\
-2.82197200 \\
-3.68844800 \\
-2.56333600 \\
-1.93930700 \\
-2.94260100 \\
-3.44226600 \\
\end{array}$ & $\begin{array}{l} \\
98.362169 \\
-1.13544800 \\
-1.17688200 \\
-2.09324100 \\
0.06103800 \\
1.26380100 \\
2.15251500 \\
1.35273400 \\
2.18885900 \\
0.30365500 \\
-0.23160000 \\
0.01407700 \\
0.30325500 \\
-1.33988000 \\
1.34444000 \\
2.21261100 \\
1.62321500 \\
1.17025800 \\
-1.36312300 \\
-2.14820900 \\
-1.75726700 \\
-1.21755600 \\
\end{array}$ & $\begin{array}{r}0.67737900 \\
1.46553000 \\
0.47665800 \\
0.34226800 \\
0.12002900 \\
-0.12162100 \\
-0.14072400 \\
-0.75664500 \\
0.08072500 \\
1.01875900 \\
0.01150200 \\
-0.49054200 \\
-0.57247600 \\
-0.04739700 \\
0.46701400 \\
-1.10640300 \\
0.35628600 \\
-0.25308400 \\
-0.70002100 \\
0.70598800 \\
-0.89541300 \\
\end{array}$ \\
\hline ר & \multicolumn{4}{|c|}{$\begin{array}{l}\text { Free Energy: }-348.606819 \\
\text { Enthalpy: }-348.561333 \\
\text { ZPE Corrected Energy: }-348.571414 \\
\text { Total Energy:- } 348.6744017 \\
\text { Frequency: }-742.4825\end{array}$} \\
\hline
\end{tabular}




\begin{tabular}{|c|c|c|c|c|}
\hline & $\begin{array}{l}\mathrm{C} \\
\mathrm{H} \\
\mathrm{C} \\
\mathrm{H} \\
\mathrm{B} \\
\mathrm{H} \\
\mathrm{F} \\
\mathrm{Cl} \\
\mathrm{Cl}\end{array}$ & $\begin{array}{r}2.36969200 \\
2.73196300 \\
3.26925400 \\
3.39088300 \\
-1.24132500 \\
4.16236500 \\
2.36853900 \\
-2.17956600 \\
-2.22384600 \\
\end{array}$ & $\begin{array}{r}1.34077100 \\
2.22791400 \\
0.23882300 \\
-0.32328600 \\
0.01946400 \\
0.24820400 \\
-1.26425700 \\
-1.54360900 \\
1.55127900 \\
\end{array}$ & $\begin{array}{r}-0.04868500 \\
-0.56520200 \\
0.05891100 \\
0.97835600 \\
0.06594400 \\
-0.56685700 \\
-0.63266900 \\
-0.08583700 \\
-0.09023600\end{array}$ \\
\hline${ }^{N C}{ }_{-C N}$ & $\begin{array}{l}\mathrm{C} \\
\mathrm{H} \\
\mathrm{H} \\
\mathrm{C} \\
\mathrm{C} \\
\mathrm{H} \\
\mathrm{C} \\
\mathrm{H} \\
\mathrm{C} \\
\mathrm{H} \\
\mathrm{B} \\
\mathrm{H} \\
\mathrm{F} \\
\mathrm{C} \\
\mathrm{C} \\
\mathrm{N} \\
\mathrm{N}\end{array}$ & $\begin{array}{l}\text { y: }-504.294062 \\
504.246127 \\
\text { ted Energy: }-5 \\
y:-504.37535 \\
-724.4110 \\
0.76545500 \\
1.41305400 \\
0.28437100 \\
0.06442900 \\
0.82502400 \\
0.25234100 \\
2.19589300 \\
2.59377000 \\
3.08256700 \\
3.21210500 \\
-1.41741300 \\
3.97402900 \\
2.18527100 \\
-2.22906500 \\
-2.19984300 \\
-2.83597700 \\
-2.78356600\end{array}$ & $\begin{array}{r}-1.19871600 \\
-1.31373400 \\
-2.12636600 \\
0.04343900 \\
1.26458900 \\
2.17606600 \\
1.33763900 \\
2.23326700 \\
0.21252100 \\
-0.31526600 \\
0.02048300 \\
0.20377500 \\
-1.23511600 \\
1.32915800 \\
-1.30644100 \\
2.34225100 \\
-2.33328600\end{array}$ & $\begin{array}{r}0.52745300 \\
1.39528400 \\
0.21922000 \\
0.29194700 \\
0.16359400 \\
-0.00149500 \\
-0.04786300 \\
-0.52079100 \\
0.06698800 \\
1.00816000 \\
0.05829700 \\
-0.56142400 \\
-0.59876700 \\
-0.04835000 \\
-0.04955500 \\
-0.13284700 \\
-0.13384400\end{array}$ \\
\hline - & $\begin{array}{l}\mathrm{C} \\
\mathrm{H} \\
\mathrm{H} \\
\mathrm{C} \\
\mathrm{C} \\
\mathrm{H} \\
\mathrm{C} \\
\mathrm{H} \\
\mathrm{C} \\
\mathrm{H} \\
\mathrm{B} \\
\mathrm{H} \\
\mathrm{F}\end{array}$ & $\begin{array}{l}\text { y: }-518.456490 \\
518.413357 \\
\text { cted Energy: }-5 \\
y:-518.52861 \\
-737.7889 \\
0.32944400 \\
1.05544600 \\
-0.18326700 \\
-0.33483500 \\
0.41043200 \\
-0.16960400 \\
1.78944100 \\
2.15298300 \\
2.68233400 \\
2.76217000 \\
-1.80732600 \\
3.59567900 \\
1.79415000\end{array}$ & $\begin{array}{l} \\
18.422811 \\
74 \\
\\
-1.19623500 \\
-1.31054500 \\
-2.12028700 \\
0.03983700 \\
1.25638600 \\
2.16052700 \\
1.34049300 \\
2.22455200 \\
0.24137200 \\
-0.34629700 \\
0.03100100 \\
0.25426700 \\
-1.28949000 \\
\end{array}$ & $\begin{array}{r}0.57698500 \\
1.37455600 \\
0.31210800 \\
0.33323400 \\
0.19739000 \\
0.01222200 \\
-0.02760000 \\
-0.54858500 \\
0.09588000 \\
1.00221700 \\
-0.00593200 \\
-0.49935700 \\
-0.65276100\end{array}$ \\
\hline
\end{tabular}




\begin{tabular}{|c|c|c|c|c|}
\hline & $\begin{array}{l}F \\
F\end{array}$ & $\begin{array}{l}-2.53481100 \\
-2.53019100\end{array}$ & $\begin{array}{r}-1.13392800 \\
1.18915700\end{array}$ & $\begin{array}{l}-0.12185300 \\
-0.18970200\end{array}$ \\
\hline 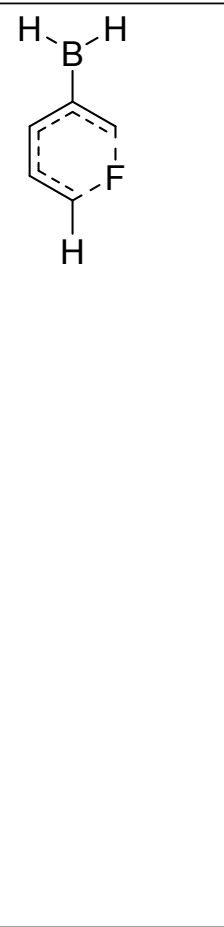 & $\begin{array}{l}\mathrm{C} \\
\mathrm{H} \\
\mathrm{H} \\
\mathrm{C} \\
\mathrm{C} \\
\mathrm{H} \\
\mathrm{C} \\
\mathrm{H} \\
\mathrm{C} \\
\mathrm{H} \\
\mathrm{B} \\
\mathrm{H} \\
\mathrm{F} \\
\mathrm{H} \\
\mathrm{H}\end{array}$ & $\begin{array}{l}y:-319.800425 \\
319.762024 \\
\text { ted Energy: }-3 \\
y:-319.88710 \\
-725.4285 \\
-0.52151700 \\
0.10052700 \\
-1.01106300 \\
-1.14613400 \\
-0.35755900 \\
-0.90814100 \\
1.03654200 \\
1.46439300 \\
1.89045500 \\
1.86430600 \\
-2.60812400 \\
2.86309100 \\
1.07245900 \\
-3.19651700 \\
-3.19883500\end{array}$ & $\begin{array}{l} \\
19.769937 \\
78 \\
-1.17455900 \\
-1.31046600 \\
-2.08707800 \\
0.07475900 \\
1.27374500 \\
2.18574300 \\
1.32872500 \\
2.18882000 \\
0.21707800 \\
-0.33207100 \\
0.08408600 \\
0.19811400 \\
-1.30721600 \\
-0.93875500 \\
1.12171700\end{array}$ & $\begin{array}{r}0.48188500 \\
1.36305900 \\
0.14266000 \\
0.17047200 \\
0.08608000 \\
-0.14822300 \\
-0.03482300 \\
-0.54724900 \\
0.20970800 \\
1.14371500 \\
-0.23900200 \\
-0.28247200 \\
-0.57531500 \\
-0.44310000 \\
-0.33548200\end{array}$ \\
\hline $\mathrm{H}_{2} \mathrm{~N}_{-}-\mathrm{NH}_{2}$ & $\begin{array}{l}\mathrm{C} \\
\mathrm{H} \\
\mathrm{H} \\
\mathrm{C} \\
\mathrm{C} \\
\mathrm{H} \\
\mathrm{C} \\
\mathrm{H} \\
\mathrm{C} \\
\mathrm{H} \\
\mathrm{B} \\
\mathrm{H} \\
\mathrm{F} \\
\mathrm{N} \\
\mathrm{H} \\
\mathrm{H} \\
\mathrm{N} \\
\mathrm{H} \\
\mathrm{H}\end{array}$ & $\begin{array}{r}0.35168000 \\
1.16533700 \\
-0.13664700 \\
-0.33035300 \\
0.37767100 \\
-0.23036500 \\
1.76281400 \\
2.05135400 \\
2.72171000 \\
2.76836800 \\
-1.85701300 \\
3.65850700 \\
1.91781100 \\
-2.32176700 \\
-1.67925500 \\
-3.29573200 \\
-2.73870700 \\
-3.70723200 \\
-2.44738200 \\
\end{array}$ & $\begin{array}{l}\text { 30.545982 } \\
15 \\
\\
-0.97081500 \\
-0.86693700 \\
-1.94392900 \\
0.12335100 \\
1.27180100 \\
2.07441500 \\
1.35127700 \\
2.06570400 \\
0.40619200 \\
-0.02124600 \\
-0.02320700 \\
0.35049600 \\
-1.44652900 \\
-1.24780000 \\
-1.96043200 \\
-1.44448600 \\
1.07738100 \\
1.09346700 \\
1.88982900 \\
\end{array}$ & $\begin{array}{r}0.93149400 \\
1.63557000 \\
0.96518800 \\
0.36980400 \\
-0.08334500 \\
-0.50630300 \\
-0.36474400 \\
-1.13569200 \\
0.03274600 \\
1.02220800 \\
-0.01396400 \\
-0.52108200 \\
-0.35230300 \\
-0.58210000 \\
-0.90345100 \\
-0.77545700 \\
0.22056500 \\
-0.07317000 \\
0.74774500 \\
\end{array}$ \\
\hline
\end{tabular}




\begin{tabular}{|c|c|c|c|c|}
\hline - & $\begin{array}{l}\mathrm{C} \\
\mathrm{H} \\
\mathrm{H} \\
\mathrm{C} \\
\mathrm{C} \\
\mathrm{H} \\
\mathrm{C} \\
\mathrm{H} \\
\mathrm{C} \\
\mathrm{H} \\
\mathrm{B} \\
\mathrm{H} \\
\mathrm{F} \\
\mathrm{O} \\
\mathrm{H} \\
\mathrm{O} \\
\mathrm{H}\end{array}$ & $\begin{array}{l}y:-470.35644 \\
170.312284 \\
\text { ted Energy: } \\
y:-470.45161 \\
-698.7610 \\
\\
0.32733500 \\
1.10759900 \\
-0.20269800 \\
-0.32288800 \\
0.41456500 \\
-0.18019000 \\
1.79898600 \\
2.13172100 \\
2.71052100 \\
2.75665100 \\
-1.82308400 \\
3.64196700 \\
1.84825200 \\
-2.47993500 \\
-3.41235700 \\
-2.46042600 \\
-3.40976900\end{array}$ & $\begin{array}{l} \\
70.322455 \\
02 \\
-1.19601300 \\
-1.31582700 \\
-2.11297400 \\
0.03613100 \\
1.24687300 \\
2.13966500 \\
1.34858200 \\
2.22827300 \\
0.28052400 \\
-0.34892500 \\
0.03120400 \\
0.31972800 \\
-1.31921000 \\
-1.19644700 \\
-1.28341600 \\
1.25432000 \\
1.33078600 \\
\end{array}$ & $\begin{array}{r}0.60579100 \\
1.34648200 \\
0.35457500 \\
0.36316600 \\
0.21322600 \\
0.02025500 \\
-0.02738400 \\
-0.57717000 \\
0.10830700 \\
0.98582400 \\
0.00506000 \\
-0.45652400 \\
-0.70171400 \\
-0.07766300 \\
-0.33761500 \\
-0.20104800 \\
-0.39464400 \\
\end{array}$ \\
\hline $\mathrm{HO}_{\mathrm{B}^{-}}-\mathrm{OH}$ & $\begin{array}{l}\text { Fre } \\
\text { En } \\
\text { ZP } \\
\text { Tot } \\
\text { C } \\
\mathrm{H} \\
\mathrm{H} \\
\mathrm{C} \\
\mathrm{C} \\
\mathrm{H} \\
\mathrm{C} \\
\mathrm{H} \\
\mathrm{C} \\
\mathrm{H} \\
\mathrm{B} \\
\mathrm{H} \\
\mathrm{O} \\
\mathrm{H} \\
\mathrm{O} \\
\mathrm{H} \\
\mathrm{Br}\end{array}$ & $\begin{array}{r}-3.05261600 \\
-3.14032600 \\
-3.85597100 \\
-1.97435200 \\
-0.92632200 \\
-1.23141600 \\
0.34197800 \\
0.89788500 \\
1.09543100 \\
0.47285300 \\
-2.04112600 \\
1.83660100 \\
-2.89304000 \\
-3.03876600 \\
-1.33813100 \\
-1.40603200 \\
2.30329900\end{array}$ & $\begin{array}{l}83.710915 \\
33 \\
1.23008300 \\
2.28840100 \\
0.57995600 \\
0.73925900 \\
1.72570000 \\
2.74957000 \\
1.62343200 \\
2.55835000 \\
0.41771300 \\
-0.43343300 \\
-0.79212000 \\
0.63532200 \\
-1.56346600 \\
-2.51374000 \\
-1.33392100 \\
-2.27353200 \\
-0.31052300\end{array}$ & $\begin{array}{r}-0.83315200 \\
-1.08196000 \\
-1.16658500 \\
-0.14873500 \\
0.20380200 \\
-0.03309900 \\
0.69544800 \\
0.77390200 \\
1.12261100 \\
1.37503100 \\
0.18571100 \\
1.89173100 \\
-0.59564300 \\
-0.44983100 \\
1.25603900 \\
1.50079000 \\
-0.43604400\end{array}$ \\
\hline 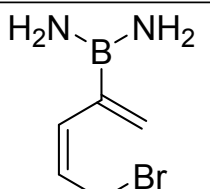 & \multicolumn{4}{|c|}{$\begin{array}{l}\text { Free Energy: }-343.981841 \\
\text { Enthalpy: -343.932102 } \\
\text { ZPE Corrected Energy: }-343.943678 \\
\text { Total Energy: }-344.0998024\end{array}$} \\
\hline
\end{tabular}




\begin{tabular}{|c|c|c|c|c|}
\hline & $\begin{array}{l}\mathrm{C} \\
\mathrm{H} \\
\mathrm{H} \\
\mathrm{C} \\
\mathrm{C} \\
\mathrm{H} \\
\mathrm{C} \\
\mathrm{H} \\
\mathrm{C} \\
\mathrm{H} \\
\mathrm{B} \\
\mathrm{H} \\
\mathrm{N} \\
\mathrm{H} \\
\mathrm{H} \\
\mathrm{N} \\
\mathrm{H} \\
\mathrm{H} \\
\mathrm{Br}\end{array}$ & $\begin{array}{r}-3.39913200 \\
-3.59842000 \\
-4.24024400 \\
-2.16317900 \\
-1.10301800 \\
-1.40347600 \\
0.15816200 \\
0.74638000 \\
0.84375600 \\
0.23783800 \\
-1.97490400 \\
1.36897300 \\
-2.87664100 \\
-3.56893500 \\
-2.88297000 \\
-0.95905200 \\
-0.80393600 \\
-0.31082700 \\
2.43315600\end{array}$ & $\begin{array}{r}1.10923500 \\
2.16595000 \\
0.43327100 \\
0.65822000 \\
1.68162400 \\
2.67339300 \\
1.61540700 \\
2.53308900 \\
0.45441100 \\
-0.44008700 \\
-0.91056600 \\
0.71428600 \\
-1.59814400 \\
-1.09685100 \\
-2.60219700 \\
-1.59847100 \\
-2.59764600 \\
-1.11456300 \\
-0.19579700\end{array}$ & $\begin{array}{r}-0.59346300 \\
-0.78118100 \\
-0.73365700 \\
-0.22518500 \\
-0.08884400 \\
-0.44048100 \\
0.42957000 \\
0.41391400 \\
1.05260500 \\
1.16685400 \\
-0.04165000 \\
1.97256900 \\
0.82590800 \\
1.36638100 \\
0.95734600 \\
-0.76294300 \\
-0.70775800 \\
-1.37142200 \\
-0.15780500\end{array}$ \\
\hline & $\begin{array}{l}\mathrm{C} \\
\mathrm{H} \\
\mathrm{H} \\
\mathrm{C} \\
\mathrm{C} \\
\mathrm{H} \\
\mathrm{C} \\
\mathrm{H} \\
\mathrm{C} \\
\mathrm{H} \\
\mathrm{B} \\
\mathrm{H} \\
\mathrm{H} \\
\mathrm{H} \\
\mathrm{Br}\end{array}$ & $\begin{array}{r}-3.07175600 \\
-3.84427700 \\
-3.30208100 \\
-1.88759600 \\
-1.63372600 \\
-2.45601500 \\
-0.47820300 \\
-0.44316200 \\
0.79257300 \\
0.70504600 \\
-0.94741400 \\
1.62473400 \\
-0.93058400 \\
-0.46827800 \\
1.47211200\end{array}$ & $\begin{array}{l} \\
33.153277 \\
27 \\
-0.82640000 \\
-0.21833400 \\
-1.88248100 \\
-0.29247300 \\
1.13292700 \\
1.66264900 \\
1.83164700 \\
2.85399600 \\
1.35687600 \\
1.08195500 \\
-1.21424200 \\
2.04370900 \\
-2.38907600 \\
-0.81924900 \\
-0.44221200\end{array}$ & $\begin{array}{r}-0.34069500 \\
-0.81587600 \\
-0.21426000 \\
0.09026700 \\
-0.20530400 \\
-0.69390600 \\
-0.03354400 \\
-0.41012600 \\
0.59410300 \\
1.64536900 \\
0.94100600 \\
0.43771400 \\
0.72887900 \\
1.96149100 \\
-0.22780800\end{array}$ \\
\hline$\underbrace{N C}_{B r}$ & $\begin{array}{l}\mathrm{C} \\
\mathrm{H} \\
\mathrm{H} \\
\mathrm{C} \\
\mathrm{C} \\
\mathrm{H} \\
\mathrm{C}\end{array}$ & $\begin{array}{l}y:-417.67086 \\
17.619212 \\
\text { ted Energy: }-4 \\
y:-417.74869 \\
-2.09666500 \\
-2.08721400 \\
-3.00845800 \\
-1.02304400 \\
0.19202600 \\
0.04627500 \\
1.46634000\end{array}$ & $\begin{array}{l} \\
17.631866 \\
31 \\
1.87284400 \\
2.87092100 \\
1.29694400 \\
1.38413300 \\
2.22485000 \\
3.26598900 \\
1.86562900 \\
\end{array}$ & $\begin{array}{r}-1.04840300 \\
-1.48796500 \\
-1.18686600 \\
-0.35619800 \\
-0.27046600 \\
-0.56519800 \\
0.03564700\end{array}$ \\
\hline
\end{tabular}




\begin{tabular}{|c|c|c|c|c|}
\hline & $\begin{array}{l}\mathrm{H} \\
\mathrm{C} \\
\mathrm{H} \\
\mathrm{B} \\
\mathrm{H} \\
\mathrm{C} \\
\mathrm{C} \\
\mathrm{N} \\
\mathrm{N} \\
\mathrm{Br}\end{array}$ & $\begin{array}{r}2.22778600 \\
2.02178400 \\
1.81717400 \\
-1.19694600 \\
3.08851500 \\
-2.20023000 \\
-0.73752600 \\
-0.39194100 \\
-2.97949200 \\
1.19327300\end{array}$ & $\begin{array}{r}2.64319200 \\
0.53464100 \\
0.26307300 \\
0.02170100 \\
0.45053600 \\
-1.03138900 \\
-0.12906400 \\
-0.20781700 \\
-1.82632500 \\
-1.05685800\end{array}$ & $\begin{array}{r}-0.03768100 \\
0.44477800 \\
1.48278100 \\
0.38782200 \\
0.23294900 \\
-0.12949300 \\
1.85716300 \\
2.98610900 \\
-0.52991400 \\
-0.59339000\end{array}$ \\
\hline $\mathrm{Cl}_{\mathrm{B}^{-}}-\mathrm{Cl}$ & $\begin{array}{l}\text { Fre } \\
\text { En } \\
\text { ZP } \\
\text { To } \\
\\
\mathrm{C} \\
\mathrm{H} \\
\mathrm{H} \\
\mathrm{C} \\
\mathrm{C} \\
\mathrm{H} \\
\mathrm{C} \\
\mathrm{H} \\
\mathrm{C} \\
\mathrm{H} \\
\mathrm{B} \\
\mathrm{H} \\
\mathrm{Cl} \\
\mathrm{Cl} \\
\mathrm{Br}\end{array}$ & $\begin{array}{l}y:-261.988646 \\
261.937555 \\
\text { ted Energy: }-2 \\
y:-262.05128 \\
-1.89095400 \\
-1.76613400 \\
-2.69521200 \\
-1.07178500 \\
0.01619600 \\
-0.15348000 \\
1.21589100 \\
1.88000000 \\
1.79894100 \\
1.21669200 \\
-1.45059500 \\
2.83469800 \\
-1.34282900 \\
-2.24471200 \\
1.90042500\end{array}$ & $\begin{array}{l} \\
61.948939 \\
1.51507600 \\
2.44072600 \\
0.85991300 \\
1.20627500 \\
2.16714800 \\
3.16641400 \\
1.98221200 \\
2.84707500 \\
0.75627200 \\
0.36195800 \\
-0.04239300 \\
0.91161100 \\
-0.04200400 \\
-1.48879800 \\
-0.86040000\end{array}$ & $\begin{array}{r}-1.71451400 \\
-2.27653100 \\
-2.03795800 \\
-0.66222400 \\
-0.34705300 \\
-0.75399600 \\
0.26527700 \\
0.28293700 \\
0.89784900 \\
1.73010800 \\
0.17491100 \\
1.19858600 \\
1.98858900 \\
-0.57162000 \\
-0.39263400\end{array}$ \\
\hline$\underbrace{\mathrm{H}_{3} \mathrm{C}_{\mathrm{B}}^{-\mathrm{CH}_{3}}}_{B r}$ & $\begin{array}{l}\mathrm{C} \\
\mathrm{H} \\
\mathrm{H} \\
\mathrm{C} \\
\mathrm{C} \\
\mathrm{H} \\
\mathrm{C} \\
\mathrm{H} \\
\mathrm{C} \\
\mathrm{H} \\
\mathrm{B} \\
\mathrm{H} \\
\mathrm{C} \\
\mathrm{H} \\
\mathrm{H} \\
\mathrm{H}\end{array}$ & $\begin{array}{r}-2.86123100 \\
-2.84828800 \\
-3.64884500 \\
-1.95730300 \\
-0.91056700 \\
-1.11963700 \\
0.28667200 \\
0.93380800 \\
0.85697000 \\
0.19550300 \\
-2.23007800 \\
1.29287600 \\
-2.31342400 \\
-3.38786400 \\
-1.92224800 \\
-1.84940300 \\
\end{array}$ & $\begin{array}{l} \\
11.749791 \\
62 \\
1.45954800 \\
2.53941200 \\
0.91664500 \\
0.83383900 \\
1.67724200 \\
2.75036600 \\
1.30340900 \\
2.08624800 \\
-0.06902100 \\
-0.86153100 \\
-0.68178900 \\
-0.31887100 \\
-1.14940600 \\
-1.20617900 \\
-2.16609100 \\
-0.46884200 \\
\end{array}$ & $\begin{array}{r}-1.08384800 \\
-1.24074200 \\
-1.60233500 \\
-0.26997300 \\
0.35380700 \\
0.35187800 \\
0.88349200 \\
1.27799200 \\
0.94343800 \\
0.60199800 \\
0.07550500 \\
1.91176500 \\
1.58810700 \\
1.83912100 \\
1.73756700 \\
2.31051000\end{array}$ \\
\hline
\end{tabular}




\begin{tabular}{|c|c|c|c|c|}
\hline & $\begin{array}{l}\mathrm{C} \\
\mathrm{H} \\
\mathrm{H} \\
\mathrm{H} \\
\mathrm{Br}\end{array}$ & $\begin{array}{l}-2.51724800 \\
-1.54488300 \\
-3.14394300 \\
-2.94084000 \\
2.50374100\end{array}$ & $\begin{array}{l}-1.72883400 \\
-2.15912400 \\
-2.56927500 \\
-1.28327200 \\
-0.22317700\end{array}$ & $\begin{array}{l}-1.07434800 \\
-1.37623400 \\
-0.74894300 \\
-1.98339400 \\
-0.32859300\end{array}$ \\
\hline$\underbrace{B r}_{B r}$ & $\begin{array}{l}\text { Fr } \\
\text { En } \\
\text { ZP } \\
\text { To } \\
\text { C } \\
\mathrm{H} \\
\mathrm{H} \\
\mathrm{C} \\
\mathrm{C} \\
\mathrm{H} \\
\mathrm{C} \\
\mathrm{H} \\
\mathrm{C} \\
\mathrm{H} \\
\mathrm{B} \\
\mathrm{H} \\
\mathrm{Br} \\
\mathrm{Br} \\
\mathrm{Br}\end{array}$ & $\begin{array}{r}0.99202400 \\
0.51090800 \\
1.84845400 \\
0.53511100 \\
-0.64981300 \\
-0.69873000 \\
-1.67624500 \\
-2.46828200 \\
-1.87274900 \\
-1.09713300 \\
1.38080000 \\
-2.05999200 \\
2.40383000 \\
1.57509100 \\
-3.60489800\end{array}$ & $\begin{array}{l} \\
58.375361 \\
11 \\
-1.29168100 \\
-1.45860900 \\
-1.92127600 \\
-0.33467700 \\
0.47290800 \\
0.71699300 \\
0.90227800 \\
1.47922600 \\
0.63691300 \\
0.03034600 \\
-0.02394000 \\
1.53973900 \\
-1.43179700 \\
1.82276800 \\
-0.46471800\end{array}$ & $\begin{array}{r}2.44816600 \\
3.41191500 \\
2.22115200 \\
1.58379600 \\
1.99310600 \\
3.05535500 \\
1.21658100 \\
1.69253200 \\
-0.23832200 \\
-0.70297700 \\
0.31936400 \\
-0.82162700 \\
-0.62130200 \\
-0.35868900 \\
-0.51924100\end{array}$ \\
\hline (c) & $\begin{array}{l}\text { Fre } \\
\text { En } \\
\text { ZP } \\
\text { To } \\
\text { Fre } \\
\text { C } \\
\mathrm{H} \\
\mathrm{H} \\
\mathrm{C} \\
\mathrm{C} \\
\mathrm{H} \\
\mathrm{C} \\
\mathrm{H} \\
\mathrm{C} \\
\mathrm{H} \\
\mathrm{H} \\
\mathrm{B} \\
\mathrm{O} \\
\mathrm{H} \\
\mathrm{O} \\
\mathrm{H} \\
\mathrm{Br}\end{array}$ & $\begin{array}{l}\text { : }-383.705996 \\
83.659251 \\
\text { ted Energy: }-3 \\
y:-383.794751 \\
-1413.3306 \\
1.10819900 \\
0.73215000 \\
1.52036200 \\
1.78322000 \\
1.04356300 \\
1.56052300 \\
-0.36351400 \\
-0.85589300 \\
-1.13503200 \\
-1.30151600 \\
-0.16735200 \\
3.20639900 \\
3.93461400 \\
3.57431800 \\
3.80118300 \\
4.70330800 \\
-2.92294000\end{array}$ & $\begin{array}{l} \\
83.669587 \\
9 \\
1.32412700 \\
1.48534700 \\
2.25086300 \\
0.08370600 \\
-1.11996000 \\
-2.06201000 \\
-1.11952600 \\
-2.02186100 \\
0.08135200 \\
0.56892600 \\
1.02706500 \\
0.02689100 \\
1.14754000 \\
2.03992400 \\
-1.20556100 \\
-1.22698500 \\
0.07915000\end{array}$ & $\begin{array}{r}0.70405100 \\
1.71865500 \\
0.29071900 \\
0.42591200 \\
0.53447200 \\
0.37368800 \\
0.50366900 \\
0.14586400 \\
0.62897600 \\
1.58772300 \\
0.25110100 \\
-0.21047400 \\
-0.58981400 \\
-0.45253800 \\
-0.39913800 \\
-0.76774500 \\
-0.31331300\end{array}$ \\
\hline
\end{tabular}




\begin{tabular}{|c|c|c|c|c|}
\hline ir & $\begin{array}{l}\mathrm{C} \\
\mathrm{H} \\
\mathrm{H} \\
\mathrm{C} \\
\mathrm{C} \\
\mathrm{H} \\
\mathrm{C} \\
\mathrm{H} \\
\mathrm{C} \\
\mathrm{H} \\
\mathrm{H} \\
\mathrm{B} \\
\mathrm{N} \\
\mathrm{H} \\
\mathrm{H} \\
\mathrm{N} \\
\mathrm{H} \\
\mathrm{H} \\
\mathrm{Br}\end{array}$ & $\begin{array}{r}-1.11273600 \\
-0.72740600 \\
-1.55420900 \\
-1.79249100 \\
-1.05016100 \\
-1.54697400 \\
0.35994400 \\
0.84962400 \\
1.13549200 \\
1.30730500 \\
0.16216100 \\
-3.23255900 \\
-3.58923000 \\
-4.50570200 \\
-2.92281100 \\
-4.15074100 \\
-3.93251000 \\
-5.08196400 \\
2.94442200\end{array}$ & $\begin{array}{l} \\
43.892288 \\
39 \\
\\
-0.97588900 \\
-0.73625300 \\
-1.97565200 \\
0.06847100 \\
1.22468700 \\
2.04097100 \\
1.21701500 \\
1.92294600 \\
0.15030500 \\
0.04516000 \\
-0.87419600 \\
-0.14676800 \\
-1.41544800 \\
-1.62535100 \\
-2.17091900 \\
0.94717000 \\
1.82127900 \\
0.90411000 \\
-0.15565300\end{array}$ & $\begin{array}{r}1.14863000 \\
2.14474700 \\
1.13213900 \\
0.42997400 \\
0.08543700 \\
-0.43592500 \\
0.05888900 \\
-0.60968700 \\
0.61614400 \\
1.68548400 \\
0.61363400 \\
-0.18990200 \\
-0.73689700 \\
-1.11186500 \\
-0.82329700 \\
-0.17559100 \\
0.28367000 \\
-0.57035400 \\
-0.25731600\end{array}$ \\
\hline 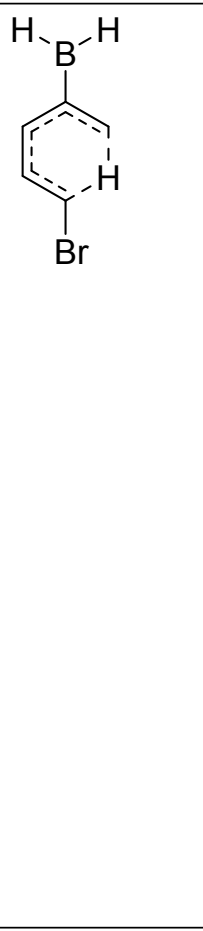 & $\begin{array}{l}\mathrm{C} \\
\mathrm{H} \\
\mathrm{H} \\
\mathrm{C} \\
\mathrm{C} \\
\mathrm{H} \\
\mathrm{C} \\
\mathrm{H} \\
\mathrm{C} \\
\mathrm{H} \\
\mathrm{H} \\
\mathrm{B} \\
\mathrm{H} \\
\mathrm{H} \\
\mathrm{Br}\end{array}$ & $\begin{array}{l}\text { y: }-233.14293 \\
33.101713 \\
\text { ted Energy: }- \\
y:-233.22214 \\
-1339.1575 \\
1.94151700 \\
1.71541100 \\
2.28583200 \\
2.59500400 \\
1.88299000 \\
2.38771100 \\
0.48265300 \\
-0.04701400 \\
-0.27319800 \\
-0.33915000 \\
0.63771300 \\
3.90793100 \\
4.26193800 \\
4.59893900 \\
-2.13756600\end{array}$ & $\begin{array}{l} \\
33.110083 \\
65 \\
1.33248600 \\
1.51299100 \\
2.23755300 \\
0.08961000 \\
-1.12451100 \\
-2.07102900 \\
-1.13571600 \\
-2.04149900 \\
0.06680700 \\
0.55224800 \\
1.02100000 \\
0.08101700 \\
1.05469700 \\
-0.89576900 \\
0.08150500 \\
\end{array}$ & $\begin{array}{r}0.37900300 \\
1.43501500 \\
-0.12707800 \\
0.03329700 \\
0.25204700 \\
0.06780200 \\
0.36819600 \\
0.07997500 \\
0.57454300 \\
1.54597900 \\
0.09172600 \\
-0.76254700 \\
-1.35983600 \\
-0.77796700 \\
-0.19386800 \\
\end{array}$ \\
\hline
\end{tabular}




\begin{tabular}{|c|c|c|c|c|}
\hline $\mathrm{F}_{\mathrm{B}^{-}} \mathrm{F}^{\mathrm{F}}$ & $\begin{array}{l}\mathrm{C} \\
\mathrm{H} \\
\mathrm{H} \\
\mathrm{C} \\
\mathrm{C} \\
\mathrm{H} \\
\mathrm{C} \\
\mathrm{H} \\
\mathrm{C} \\
\mathrm{H} \\
\mathrm{H} \\
\mathrm{B} \\
\mathrm{F} \\
\mathrm{F} \\
\mathrm{Br}\end{array}$ & $\begin{array}{l}y:-431.79955 \\
\text { 31.753613 } \\
\text { ted Energy: } \\
\text { y: }-431.8644 \\
-1372.4833 \\
\\
-1.11134600 \\
-0.74868000 \\
-1.52749700 \\
-1.77839300 \\
-1.04600300 \\
-1.55421400 \\
0.35824600 \\
0.86171700 \\
1.12250000 \\
1.28348600 \\
0.16170700 \\
-3.16678800 \\
-3.88289200 \\
-3.79265600 \\
2.89049500\end{array}$ & $\begin{array}{l}3 \\
\\
-1.26671300 \\
-1.36930500 \\
-2.20018500 \\
-0.04029900 \\
1.17540900 \\
2.11518800 \\
1.16939400 \\
2.05286900 \\
-0.03132100 \\
-0.47080900 \\
-0.99322000 \\
-0.05861200 \\
1.08875800 \\
-1.22438400 \\
-0.10456200 \\
\end{array}$ & $\begin{array}{r}0.77133100 \\
1.79790500 \\
0.38860100 \\
0.42021600 \\
0.47593100 \\
0.27421300 \\
0.46097300 \\
0.07403900 \\
0.64616300 \\
1.62854500 \\
0.31158900 \\
-0.21714200 \\
-0.44749000 \\
-0.57577300 \\
-0.30935700 \\
\end{array}$ \\
\hline $\mathrm{NC}_{-}{ }_{-\mathrm{CN}}$ & $\begin{array}{l}\text { Fre } \\
\text { En } \\
\text { ZP } \\
\text { Tot } \\
\text { Fre } \\
\text { C } \\
\mathrm{H} \\
\mathrm{H} \\
\mathrm{C} \\
\mathrm{C} \\
\mathrm{H} \\
\mathrm{C} \\
\mathrm{H} \\
\mathrm{C} \\
\mathrm{H} \\
\mathrm{H} \\
\mathrm{B} \\
\mathrm{C} \\
\mathrm{C} \\
\mathrm{N} \\
\mathrm{N} \\
\mathrm{Br}\end{array}$ & $\begin{array}{l}y:-417.63138 \\
\text { t17.580387 } \\
\text { ted Energy: } \\
y:-417.70470 \\
-1274.0420 \\
\\
0.64837500 \\
0.28553100 \\
1.02729400 \\
1.35334400 \\
0.61992900 \\
1.14133100 \\
-0.77607400 \\
-1.26830200 \\
-1.55755900 \\
-1.77934500 \\
-0.59948300 \\
2.76267200 \\
3.42035200 \\
3.59981400 \\
3.91442800 \\
4.23015500 \\
-3.24233000\end{array}$ & $\begin{array}{l}7 \\
71.592339 \\
17 \\
1.14648500 \\
1.13677900 \\
2.12686900 \\
-0.02798000 \\
-1.25383600 \\
-2.17353000 \\
-1.25034300 \\
-2.10077900 \\
-0.06949900 \\
0.25117800 \\
0.91689400 \\
0.06675000 \\
1.42650400 \\
-1.19730900 \\
2.47676900 \\
-2.17742900 \\
0.13626600 \\
\end{array}$ & $\begin{array}{r} \\
1.01976000 \\
2.05159800 \\
0.72499500 \\
0.55431700 \\
0.47790600 \\
0.22397900 \\
0.41526600 \\
-0.05143300 \\
0.68618500 \\
1.70257700 \\
0.50577400 \\
-0.01561900 \\
-0.31382700 \\
-0.28840300 \\
-0.54738200 \\
-0.49726700 \\
-0.37354500 \\
\end{array}$ \\
\hline$\overbrace{\mathrm{Br}}^{\mathrm{Cl}}$ & \multicolumn{4}{|c|}{$\begin{array}{l}\text { Free Energy: }-261.948974 \\
\text { Enthalpy: }-261.900590 \\
\text { ZPE Corrected Energy: }-261.911089 \\
\text { Total Energy: }-262.0090819 \\
\text { Frequency: }-1329.6223\end{array}$} \\
\hline
\end{tabular}




\begin{tabular}{|c|c|c|c|c|}
\hline & $\begin{array}{l}\mathrm{C} \\
\mathrm{H} \\
\mathrm{H} \\
\mathrm{C} \\
\mathrm{C} \\
\mathrm{H} \\
\mathrm{C} \\
\mathrm{H} \\
\mathrm{C} \\
\mathrm{H} \\
\mathrm{H} \\
\mathrm{B} \\
\mathrm{Cl} \\
\mathrm{Cl} \\
\mathrm{Br}\end{array}$ & $\begin{array}{r}0.43671100 \\
0.04527300 \\
0.84306200 \\
1.13580800 \\
0.39080900 \\
0.90208200 \\
-1.00896700 \\
-1.49699900 \\
-1.78465400 \\
-2.00376100 \\
-0.80977500 \\
2.55001300 \\
3.54452100 \\
3.33634000 \\
-3.49208000\end{array}$ & $\begin{array}{r}1.14003400 \\
1.11511200 \\
2.11774300 \\
-0.03524300 \\
-1.24741500 \\
-2.16884700 \\
-1.23215700 \\
-2.07372200 \\
-0.05293500 \\
0.27645800 \\
0.92733200 \\
0.04918000 \\
-1.43926200 \\
1.64013100 \\
0.13461600\end{array}$ & $\begin{array}{r}1.04296500 \\
2.06385900 \\
0.78095700 \\
0.57950000 \\
0.48220200 \\
0.21705500 \\
0.40842100 \\
-0.07865700 \\
0.68013200 \\
1.69395700 \\
0.50373600 \\
0.00215600 \\
-0.30585700 \\
-0.37728500 \\
-0.36393100\end{array}$ \\
\hline $\mathrm{H}_{3} \mathrm{C}_{-}-\mathrm{CH}_{3}$ & $\begin{array}{l}\mathrm{C} \\
\mathrm{H} \\
\mathrm{H} \\
\mathrm{C} \\
\mathrm{C} \\
\mathrm{H} \\
\mathrm{C} \\
\mathrm{H} \\
\mathrm{C} \\
\mathrm{H} \\
\mathrm{H} \\
\mathrm{B} \\
\mathrm{C} \\
\mathrm{H} \\
\mathrm{H} \\
\mathrm{H} \\
\mathrm{C} \\
\mathrm{H} \\
\mathrm{H} \\
\mathrm{H} \\
\mathrm{Br}\end{array}$ & $\begin{array}{r}-1.07112800 \\
-0.70837400 \\
-1.48010700 \\
-1.76036600 \\
-1.01892100 \\
-1.52626800 \\
0.38798900 \\
0.87690700 \\
1.16405000 \\
1.34599100 \\
0.19253500 \\
-3.18456500 \\
-4.12952400 \\
-3.85369200 \\
-4.10301200 \\
-5.17725200 \\
-3.73157200 \\
-2.95478400 \\
-4.32081800 \\
-4.42172200 \\
2.94315000\end{array}$ & $\begin{array}{l}1 \\
11.705530 \\
-1.10872600 \\
-1.01855800 \\
-2.10208000 \\
0.02717100 \\
1.22645700 \\
2.11893800 \\
1.22128000 \\
2.01341600 \\
0.08572600 \\
-0.15809300 \\
-0.92622100 \\
-0.10706400 \\
1.16087900 \\
2.01536400 \\
1.49947700 \\
0.89835300 \\
-1.51043800 \\
-2.23491300 \\
-1.96303400 \\
-1.39582100 \\
-0.13787400\end{array}$ & $\begin{array}{r}1.02345500 \\
2.05260700 \\
0.82662700 \\
0.46016100 \\
0.28882200 \\
-0.07137400 \\
0.23929300 \\
-0.32476200 \\
0.64220200 \\
1.68681000 \\
0.51285500 \\
-0.17331900 \\
-0.30987900 \\
0.31961100 \\
-1.36074400 \\
-0.10612000 \\
-0.67075600 \\
-0.94597000 \\
0.14637600 \\
-1.51754700 \\
-0.29690100\end{array}$ \\
\hline - & \multicolumn{4}{|c|}{$\begin{array}{l}\text { Free Energy: -258.374643 } \\
\text { Enthalpy: -258.323664 } \\
\text { ZPE Corrected Energy: -258.334635 } \\
\text { Total Energy: -258.4314642 } \\
\text { Frequency: -1327.7727 }\end{array}$} \\
\hline
\end{tabular}




\begin{tabular}{|c|c|c|c|c|}
\hline & $\begin{array}{l}\mathrm{C} \\
\mathrm{C} \\
\mathrm{H} \\
\mathrm{C} \\
\mathrm{H} \\
\mathrm{C} \\
\mathrm{H} \\
\mathrm{H} \\
\mathrm{B} \\
\mathrm{Br} \\
\mathrm{Br} \\
\mathrm{Br}\end{array}$ & $\begin{array}{r}0.22813400 \\
-0.51611300 \\
0.00048200 \\
-1.91281500 \\
-2.38539000 \\
-2.69870400 \\
-2.95234600 \\
-1.71762800 \\
1.66229200 \\
2.79586700 \\
2.49144500 \\
-4.37082800\end{array}$ & $\begin{array}{r}-0.11826400 \\
-1.31734900 \\
-2.21902100 \\
-1.29297300 \\
-2.09450900 \\
-0.13772600 \\
0.11165300 \\
0.84990100 \\
0.01224500 \\
-1.58210500 \\
1.77662300 \\
0.13348400\end{array}$ & $\begin{array}{r}0.68060600 \\
0.46790300 \\
0.15051700 \\
0.35251600 \\
-0.21142400 \\
0.69040900 \\
1.71886700 \\
0.62463200 \\
0.15257700 \\
-0.17085600 \\
-0.20110100 \\
-0.39259700\end{array}$ \\
\hline $\mathrm{HO}_{-\mathrm{B}}$ & $\begin{array}{l}\mathrm{C} \\
\mathrm{H} \\
\mathrm{H} \\
\mathrm{C} \\
\mathrm{C} \\
\mathrm{H} \\
\mathrm{C} \\
\mathrm{H} \\
\mathrm{C} \\
\mathrm{H} \\
\mathrm{B} \\
\mathrm{H} \\
\mathrm{O} \\
\mathrm{H} \\
\mathrm{O} \\
\mathrm{H} \\
\mathrm{Br}\end{array}$ & $\begin{array}{l}\text { y: }-383.720422 \\
383.673594 \\
\text { ted Energy: }-3 \\
y:-383.81240 \varsigma \\
-289.1362 \\
-0.28897900 \\
0.44221700 \\
-0.52536500 \\
-1.17848000 \\
-0.86709100 \\
-1.67868800 \\
0.40929000 \\
0.48646200 \\
1.61292600 \\
1.67080500 \\
-2.52321600 \\
2.55085400 \\
-2.75370500 \\
-3.54177700 \\
-3.42611800 \\
-4.29584100 \\
1.96628600\end{array}$ & $\begin{array}{l} \\
83.684209 \\
93 \\
-0.51897200 \\
-0.13913700 \\
-1.57468400 \\
0.30487300 \\
1.62763300 \\
2.15440600 \\
2.22095200 \\
3.04439500 \\
1.76688700 \\
1.26983700 \\
-0.31704100 \\
2.19769500 \\
-1.65938300 \\
-2.14287200 \\
0.51084700 \\
0.21291900 \\
-0.76163700\end{array}$ & $\begin{array}{r}1.16512700 \\
1.86491300 \\
1.27056200 \\
0.45192500 \\
0.02196800 \\
-0.47897800 \\
-0.08442200 \\
-0.79522800 \\
0.47656400 \\
1.43332200 \\
-0.03224200 \\
0.13221400 \\
0.23187700 \\
-0.06716600 \\
-0.68835600 \\
-1.00394500 \\
-0.30656100\end{array}$ \\
\hline$\overbrace{-B r}^{\mathrm{H}_{2} \mathrm{~N}_{-}}$ & $\begin{array}{l}\mathrm{C} \\
\mathrm{H} \\
\mathrm{H} \\
\mathrm{C} \\
\mathrm{C} \\
\mathrm{H} \\
\mathrm{C} \\
\mathrm{H} \\
\mathrm{C} \\
\mathrm{H}\end{array}$ & $\begin{array}{r}-0.32184100 \\
0.40334600 \\
-0.50896700 \\
-1.21778900 \\
-0.93580700 \\
-1.74689800 \\
0.32624700 \\
0.37723400 \\
1.54196100 \\
1.62144200 \\
\end{array}$ & $\begin{array}{l} \\
43.910684 \\
33 \\
\\
-0.27164600 \\
0.25491000 \\
-1.29679500 \\
0.36430800 \\
1.60465600 \\
2.01965300 \\
2.21051800 \\
2.89644700 \\
1.90929200 \\
1.55624100 \\
\end{array}$ & $\begin{array}{r}1.28301300 \\
1.88818500 \\
1.59532600 \\
0.41372800 \\
-0.22946500 \\
-0.83081900 \\
-0.43984800 \\
-1.28649600 \\
0.18634600 \\
1.20263300 \\
\end{array}$ \\
\hline
\end{tabular}




\begin{tabular}{|c|c|c|c|c|}
\hline & $\begin{array}{l}\mathrm{B} \\
\mathrm{H} \\
\mathrm{N} \\
\mathrm{H} \\
\mathrm{H} \\
\mathrm{N} \\
\mathrm{H} \\
\mathrm{H} \\
\mathrm{Br}\end{array}$ & $\begin{array}{r}-2.52579400 \\
2.46424600 \\
-2.38918200 \\
-1.47692200 \\
-3.16763200 \\
-3.78314200 \\
-4.64601500 \\
-3.88959900 \\
2.00138200\end{array}$ & $\begin{array}{r}-0.42103800 \\
2.30826500 \\
-1.77811000 \\
-2.19408200 \\
-2.37854200 \\
0.25390100 \\
-0.15803500 \\
1.17400700 \\
-0.75171900\end{array}$ & $\begin{array}{r}-0.03172000 \\
-0.23056300 \\
-0.43994500 \\
-0.58211400 \\
-0.68148700 \\
-0.01321600 \\
-0.34610900 \\
0.39310200 \\
-0.14495900\end{array}$ \\
\hline - & $\begin{array}{l}\text { Fre } \\
\text { En } \\
\text { ZP } \\
\text { To } \\
\text { Fre } \\
\\
\text { C } \\
\mathrm{H} \\
\mathrm{H} \\
\mathrm{C} \\
\mathrm{C} \\
\mathrm{H} \\
\mathrm{C} \\
\mathrm{H} \\
\mathrm{C} \\
\mathrm{H} \\
\mathrm{B} \\
\mathrm{H} \\
\mathrm{H} \\
\mathrm{H} \\
\mathrm{Br}\end{array}$ & $\begin{array}{l}\text { y: }-233.159502 \\
233.118294 \\
\text { ted Energy: }-2 \\
y:-233.242607 \\
-305.8215 \\
-0.75961900 \\
-0.31514100 \\
-0.63698900 \\
-1.81279600 \\
-1.88402600 \\
-2.78190200 \\
-0.83884300 \\
-0.93966100 \\
0.39218700 \\
0.47252000 \\
-2.85673600 \\
1.19125600 \\
-2.67261800 \\
-3.87495700 \\
1.52170700 \\
\end{array}$ & $\begin{array}{r}-0.95881300 \\
-0.42819300 \\
-2.03938700 \\
-0.42006400 \\
0.96334300 \\
1.25344100 \\
1.90366400 \\
2.73998100 \\
1.79598000 \\
1.35984200 \\
-1.39126300 \\
2.49117000 \\
-2.57161100 \\
-0.97209300 \\
-0.41661400 \\
\end{array}$ & $\begin{array}{r}0.96104800 \\
1.79575300 \\
0.99849800 \\
0.17550100 \\
-0.19653700 \\
-0.74386600 \\
-0.19040300 \\
-0.88237700 \\
0.49145500 \\
1.47717700 \\
-0.40044000 \\
0.24155600 \\
-0.36761900 \\
-0.86828700 \\
-0.20271500 \\
\end{array}$ \\
\hline$\overbrace{\mathrm{H}^{-}}$ & $\begin{array}{l}\mathrm{C} \\
\mathrm{H} \\
\mathrm{H} \\
\mathrm{C} \\
\mathrm{C} \\
\mathrm{H} \\
\mathrm{C} \\
\mathrm{H} \\
\mathrm{C} \\
\mathrm{H} \\
\mathrm{B} \\
\mathrm{H} \\
\mathrm{F} \\
\mathrm{F} \\
\mathrm{Br}\end{array}$ & $\begin{array}{r}-0.25741700 \\
0.42554000 \\
-0.48620300 \\
-1.15973100 \\
-0.84923700 \\
-1.65625400 \\
0.42832700 \\
0.52098100 \\
1.62924500 \\
1.67897200 \\
-2.48691000 \\
2.57296900 \\
-2.79387000 \\
-3.46282500 \\
1.91261900 \\
\end{array}$ & $\begin{array}{r}-0.54451400 \\
-0.16654100 \\
-1.60390000 \\
0.29337500 \\
1.62428800 \\
2.17054400 \\
2.19956900 \\
3.03164600 \\
1.70940700 \\
1.26534800 \\
-0.31010200 \\
2.11870400 \\
-1.63432900 \\
0.44549100 \\
-0.75024300\end{array}$ & $\begin{array}{r}1.12363400 \\
1.87247400 \\
1.21837700 \\
0.42566500 \\
0.00658600 \\
-0.48149400 \\
-0.09644400 \\
-0.79420500 \\
0.45946800 \\
1.44379400 \\
-0.03713100 \\
0.10444500 \\
0.14182500 \\
-0.63768900 \\
-0.29224000 \\
\end{array}$ \\
\hline
\end{tabular}




\begin{tabular}{|c|c|c|c|c|}
\hline (- & $\begin{array}{l}\mathrm{C} \\
\mathrm{H} \\
\mathrm{H} \\
\mathrm{C} \\
\mathrm{C} \\
\mathrm{H} \\
\mathrm{C} \\
\mathrm{H} \\
\mathrm{C} \\
\mathrm{H} \\
\mathrm{B} \\
\mathrm{H} \\
\mathrm{C} \\
\mathrm{C} \\
\mathrm{N} \\
\mathrm{N} \\
\mathrm{Br}\end{array}$ & $\begin{array}{l}\text { : }-417.647758 \\
17.597140 \\
\text { ted Energy: }-4 \\
y:-417.72547 \\
-315.9718 \\
\\
0.14924600 \\
0.82169300 \\
-0.10496800 \\
-0.77234000 \\
-0.40431200 \\
-1.20184000 \\
0.89014900 \\
1.00279700 \\
2.09484000 \\
2.17141800 \\
-2.15538000 \\
3.03795200 \\
-3.26430100 \\
-2.52638800 \\
-4.10862700 \\
-2.80392600 \\
2.18389600\end{array}$ & $\begin{array}{l}17.609127 \\
95 \\
-0.58932000 \\
-0.30841000 \\
-1.64708700 \\
0.34833800 \\
1.71008400 \\
2.35270600 \\
2.22334800 \\
3.13090000 \\
1.60469800 \\
1.12532000 \\
-0.14578200 \\
2.00112400 \\
0.83394900 \\
-1.64034500 \\
1.59527500 \\
-2.79087000 \\
-0.70002900\end{array}$ & $\begin{array}{r}1.05721700 \\
1.85975200 \\
1.04586300 \\
0.49437300 \\
0.20787400 \\
-0.16314800 \\
0.10272800 \\
-0.48973100 \\
0.53952000 \\
1.50748400 \\
0.09124400 \\
0.16737200 \\
-0.34028900 \\
0.12660200 \\
-0.67054900 \\
0.15583200 \\
-0.39739800\end{array}$ \\
\hline $\mathrm{Cl}_{3}$ & $\begin{array}{l}\mathrm{C} \\
\mathrm{H} \\
\mathrm{H} \\
\mathrm{C} \\
\mathrm{C} \\
\mathrm{H} \\
\mathrm{C} \\
\mathrm{H} \\
\mathrm{C} \\
\mathrm{H} \\
\mathrm{B} \\
\mathrm{H} \\
\mathrm{Br} \\
\mathrm{Cl} \\
\mathrm{Cl}\end{array}$ & $\begin{array}{l}\text { y: }-261.964811 \\
61.916501 \\
\text { ted Energy: }-2 \\
y:-262.02870 \\
-319.5318 \\
\\
0.30407700 \\
1.01551700 \\
0.04236000 \\
-0.59986600 \\
-0.20977300 \\
-0.99255400 \\
1.09677600 \\
1.21140000 \\
2.29422100 \\
2.37108000 \\
-1.98634500 \\
3.23986300 \\
2.37453100 \\
-3.29331300 \\
-2.43475200\end{array}$ & $\begin{array}{l} \\
61.927118 \\
08 \\
-0.50387500 \\
-0.18296000 \\
-1.55745000 \\
0.41012700 \\
1.74787500 \\
2.38606000 \\
2.24370100 \\
3.11720000 \\
1.64688300 \\
1.16071300 \\
-0.09110900 \\
2.02448700 \\
-0.74685500 \\
1.04861600 \\
-1.84984300 \\
\end{array}$ & $\begin{array}{r}1.12118500 \\
1.87093200 \\
1.17498400 \\
0.51411400 \\
0.17322000 \\
-0.23360400 \\
0.04989800 \\
-0.59195800 \\
0.50935200 \\
1.47233900 \\
0.09302600 \\
0.12480300 \\
-0.37668400 \\
-0.45463700 \\
0.14256000\end{array}$ \\
\hline$\overbrace{-1}^{-}$ & \multicolumn{4}{|c|}{$\begin{array}{l}\text { Free Energy: }-311.760231 \\
\text { Enthalpy: }-311.710270 \\
\text { ZPE Corrected Energy: }-311.722281 \\
\text { Total Energy: }-311.8955745 \\
\text { Frequency: }-289.2880\end{array}$} \\
\hline
\end{tabular}




\begin{tabular}{|c|c|c|c|c|}
\hline & $\begin{array}{l}\mathrm{C} \\
\mathrm{H} \\
\mathrm{H} \\
\mathrm{C} \\
\mathrm{C} \\
\mathrm{H} \\
\mathrm{C} \\
\mathrm{H} \\
\mathrm{C} \\
\mathrm{H} \\
\mathrm{B} \\
\mathrm{H} \\
\mathrm{C} \\
\mathrm{H} \\
\mathrm{H} \\
\mathrm{H} \\
\mathrm{C} \\
\mathrm{H} \\
\mathrm{H} \\
\mathrm{H} \\
\mathrm{Br}\end{array}$ & $\begin{array}{r}-0.24340800 \\
0.45268400 \\
-0.42890800 \\
-1.16150300 \\
-0.84806300 \\
-1.65920600 \\
0.42899900 \\
0.49958700 \\
1.64099300 \\
1.70768600 \\
-2.51888600 \\
2.57399600 \\
-3.76225100 \\
-3.75481700 \\
-3.78380600 \\
-4.70816200 \\
-2.68302200 \\
-1.75581300 \\
-3.04361200 \\
-3.44471900 \\
1.99168700\end{array}$ & $\begin{array}{r}-0.45148900 \\
-0.02507800 \\
-1.51302500 \\
0.32547800 \\
1.63858000 \\
2.17221400 \\
2.21511800 \\
3.00154100 \\
1.78662900 \\
1.36045100 \\
-0.33013200 \\
2.19996200 \\
0.56853000 \\
1.58854500 \\
0.65113200 \\
0.08798700 \\
-1.90642200 \\
-2.45266900 \\
-2.25263500 \\
-2.21085500 \\
-0.74329900\end{array}$ & $\begin{array}{r}1.17944700 \\
1.89118400 \\
1.32139800 \\
0.44157300 \\
-0.02786600 \\
-0.52347600 \\
-0.19304400 \\
-0.94539000 \\
0.37610100 \\
1.36623300 \\
-0.00573400 \\
-0.00160800 \\
-0.41023700 \\
-0.00636000 \\
-1.51158600 \\
-0.12530900 \\
-0.05075200 \\
-0.26506500 \\
0.93423200 \\
-0.78047700 \\
-0.26332700\end{array}$ \\
\hline$\overbrace{-1}^{-B r}$ & $\begin{array}{l}\mathrm{C} \\
\mathrm{H} \\
\mathrm{H} \\
\mathrm{C} \\
\mathrm{C} \\
\mathrm{H} \\
\mathrm{C} \\
\mathrm{H} \\
\mathrm{C} \\
\mathrm{H} \\
\mathrm{B} \\
\mathrm{H} \\
\mathrm{Br} \\
\mathrm{Br} \\
\mathrm{Br}\end{array}$ & $\begin{array}{l}y:-258.390091 \\
\text { ted Energy: }-2 \\
y:-258.450563 \\
-318.6199 \\
1.08134200 \\
1.80728100 \\
0.82447200 \\
0.16186900 \\
0.55579600 \\
-0.23008600 \\
1.86273300 \\
1.96632400 \\
3.06909700 \\
3.16964300 \\
-1.24240400 \\
4.00576400 \\
-2.71038100 \\
-1.70963200 \\
3.11382900\end{array}$ & $\begin{array}{l} \\
58.350169 \\
31 \\
\\
-0.42162400 \\
-0.11600400 \\
-1.47692400 \\
0.50798800 \\
1.85262500 \\
2.51014800 \\
2.34284100 \\
3.23257000 \\
1.73013400 \\
1.21954300 \\
0.02225500 \\
2.11468500 \\
1.29320600 \\
-1.90176800 \\
-0.63906800\end{array}$ & $\begin{array}{r}1.12897500 \\
1.87143100 \\
1.16314600 \\
0.56799200 \\
0.25680700 \\
-0.11107200 \\
0.11426100 \\
-0.50707100 \\
0.52717800 \\
1.47521500 \\
0.17102600 \\
0.12772700 \\
-0.24507600 \\
0.08000100 \\
-0.41909000\end{array}$ \\
\hline$\underbrace{\prime}_{H}$ & \multicolumn{4}{|c|}{$\begin{array}{l}\text { Free Energy: -207.730148 } \\
\text { Enthalpy: -207.691619 } \\
\text { ZPE Corrected Energy: -207.698826 } \\
\text { Total Energy: -207.8031441 } \\
\text { Frequency: -293.0709 }\end{array}$} \\
\hline
\end{tabular}




\begin{tabular}{|c|c|c|c|c|}
\hline & $\begin{array}{l}\mathrm{C} \\
\mathrm{C} \\
\mathrm{H} \\
\mathrm{C} \\
\mathrm{H} \\
\mathrm{C} \\
\mathrm{H} \\
\mathrm{H} \\
\mathrm{Br} \\
\mathrm{H}\end{array}$ & $\begin{array}{r}-1.72851800 \\
-2.29539500 \\
-3.15359500 \\
-1.72854500 \\
-2.09588500 \\
-0.63980500 \\
-0.40294600 \\
-0.16907200 \\
1.44797000 \\
-2.09599000 \\
\end{array}$ & $\begin{array}{r}-1.26752900 \\
-0.00006800 \\
-0.00012500 \\
1.26744400 \\
2.09059000 \\
1.54139300 \\
0.96248100 \\
2.52082300 \\
0.00003500 \\
-2.09082000 \\
\end{array}$ & $\begin{array}{r}-0.14338800 \\
-0.41696700 \\
-1.09005800 \\
-0.14354500 \\
-0.75658300 \\
0.69080000 \\
1.57037900 \\
0.64788200 \\
-0.16853900 \\
-0.75617400 \\
\end{array}$ \\
\hline$\overbrace{B r}^{B r}$ & $\begin{array}{l}\text { Fre } \\
\text { En } \\
\text { ZP } \\
\text { To } \\
\text { C } \\
\mathrm{H} \\
\mathrm{C} \\
\mathrm{C} \\
\mathrm{H} \\
\mathrm{C} \\
\mathrm{H} \\
\mathrm{C} \\
\mathrm{H} \\
\mathrm{H} \\
\mathrm{B} \\
\mathrm{H} \\
\mathrm{Br} \\
\mathrm{Br} \\
\mathrm{Br}\end{array}$ & $\begin{array}{l}y:-258.429366 \\
58.375627 \\
\text { ted Energy: }-2 \\
y:-258.488979 \\
-1.45406500 \\
-1.04193700 \\
-0.40098300 \\
-0.70453000 \\
0.08894600 \\
-2.00262400 \\
-2.85331000 \\
-2.18792000 \\
-1.36814900 \\
-2.16666900 \\
1.01655200 \\
-3.16185200 \\
-2.65319400 \\
1.50527800 \\
2.45994300\end{array}$ & $\begin{array}{l} \\
58.387576 \\
94 \\
-0.42838600 \\
-1.34819700 \\
0.53308900 \\
1.87457200 \\
2.55142000 \\
2.50083700 \\
1.87252100 \\
3.84085700 \\
4.50570800 \\
0.01219600 \\
0.02279600 \\
4.29997500 \\
-1.11115400 \\
-1.88016600 \\
1.22179400\end{array}$ & $\begin{array}{r}0.98576500 \\
1.39486800 \\
0.50011700 \\
0.35399200 \\
0.03711100 \\
0.56357600 \\
0.81662100 \\
0.42581000 \\
0.15755600 \\
1.68276300 \\
0.17405200 \\
0.57471300 \\
-0.56306600 \\
0.39381300 \\
-0.47387400\end{array}$ \\
\hline 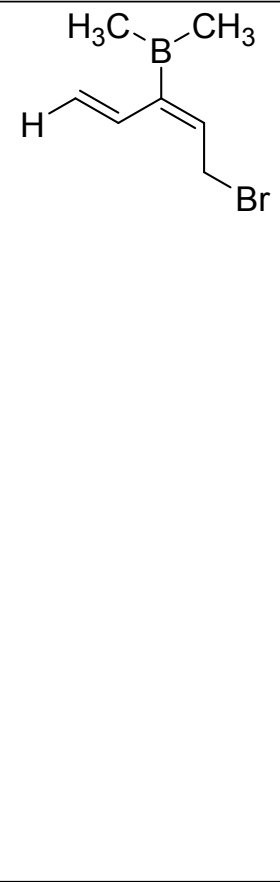 & $\begin{array}{l}\text { Fre } \\
\text { En } \\
\text { ZP } \\
\text { To } \\
\text { C } \\
\text { H } \\
\text { C } \\
\text { C } \\
\text { H } \\
\text { C } \\
\text { H } \\
\text { C } \\
\text { H } \\
\text { H } \\
\text { B } \\
\text { C } \\
\text { H } \\
\text { H } \\
\text { H }\end{array}$ & $\begin{array}{c}y:-311.798248 \\
311.745760 \\
\text { ted Energy: }-3 \\
y:-311.932916 \\
0.47893900 \\
0.67336400 \\
-0.85571900 \\
-1.43586600 \\
-2.42741000 \\
-0.85822300 \\
0.15813600 \\
-1.52652200 \\
-2.53895700 \\
0.68104900 \\
-1.56592300 \\
-3.03362900 \\
-2.95584800 \\
-3.63422100 \\
-3.59702800\end{array}$ & $\begin{array}{l} \\
11.758539 \\
64 \\
0.15588700 \\
1.08644300 \\
0.13275000 \\
-1.07288100 \\
-1.05845900 \\
-2.40571300 \\
-2.48721200 \\
-3.54027300 \\
-3.50842400 \\
-0.69892000 \\
1.49185600 \\
1.55413000 \\
1.80255000 \\
0.64140500 \\
2.38214600 \\
\end{array}$ & $\begin{array}{r}1.07445900 \\
1.60430600 \\
0.38348800 \\
0.05453300 \\
-0.39865600 \\
0.21394800 \\
0.59440800 \\
-0.12011400 \\
-0.52083600 \\
1.72077800 \\
0.02811000 \\
-0.56763000 \\
-1.64060000 \\
-0.48269900 \\
-0.11299400 \\
\end{array}$ \\
\hline
\end{tabular}




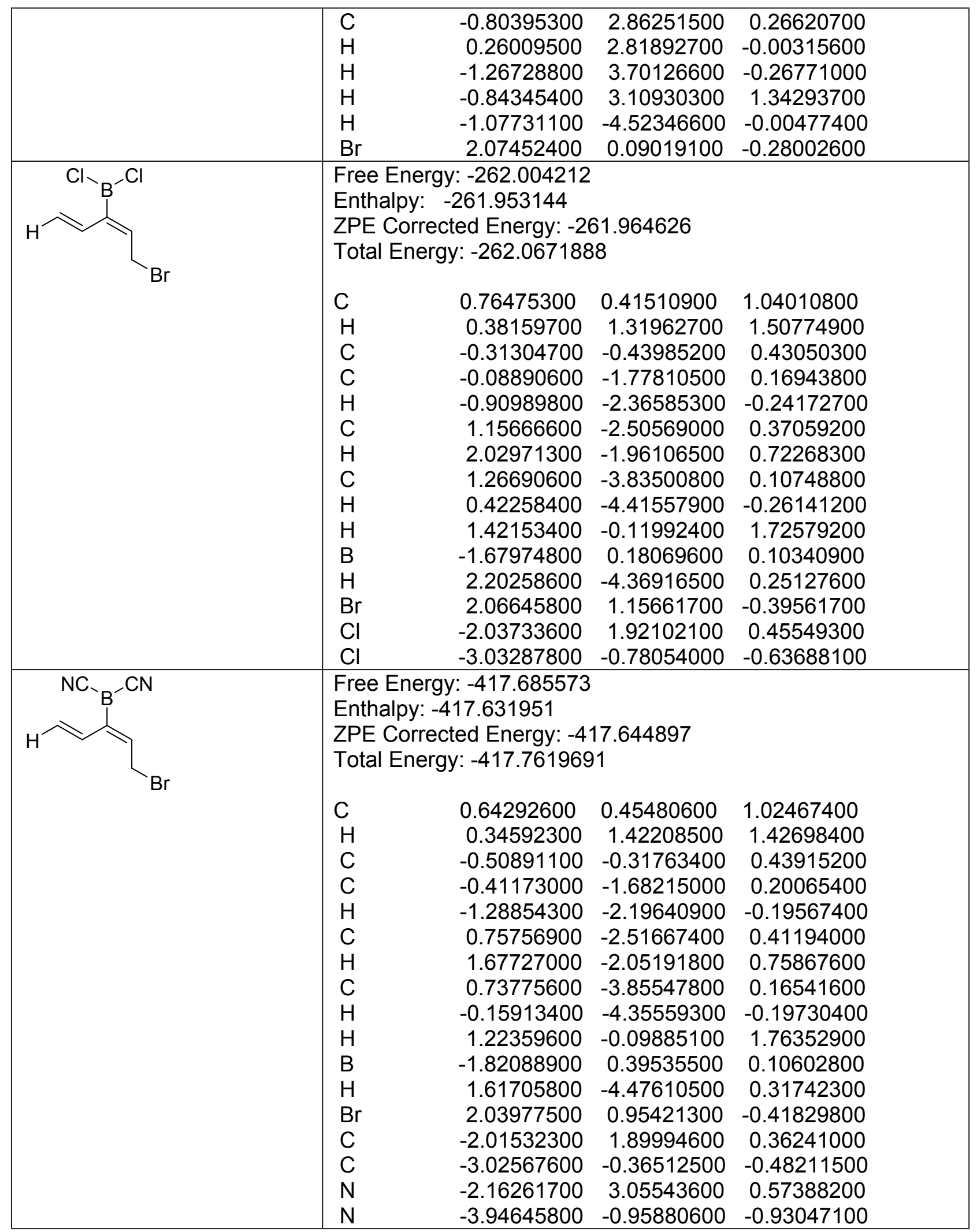




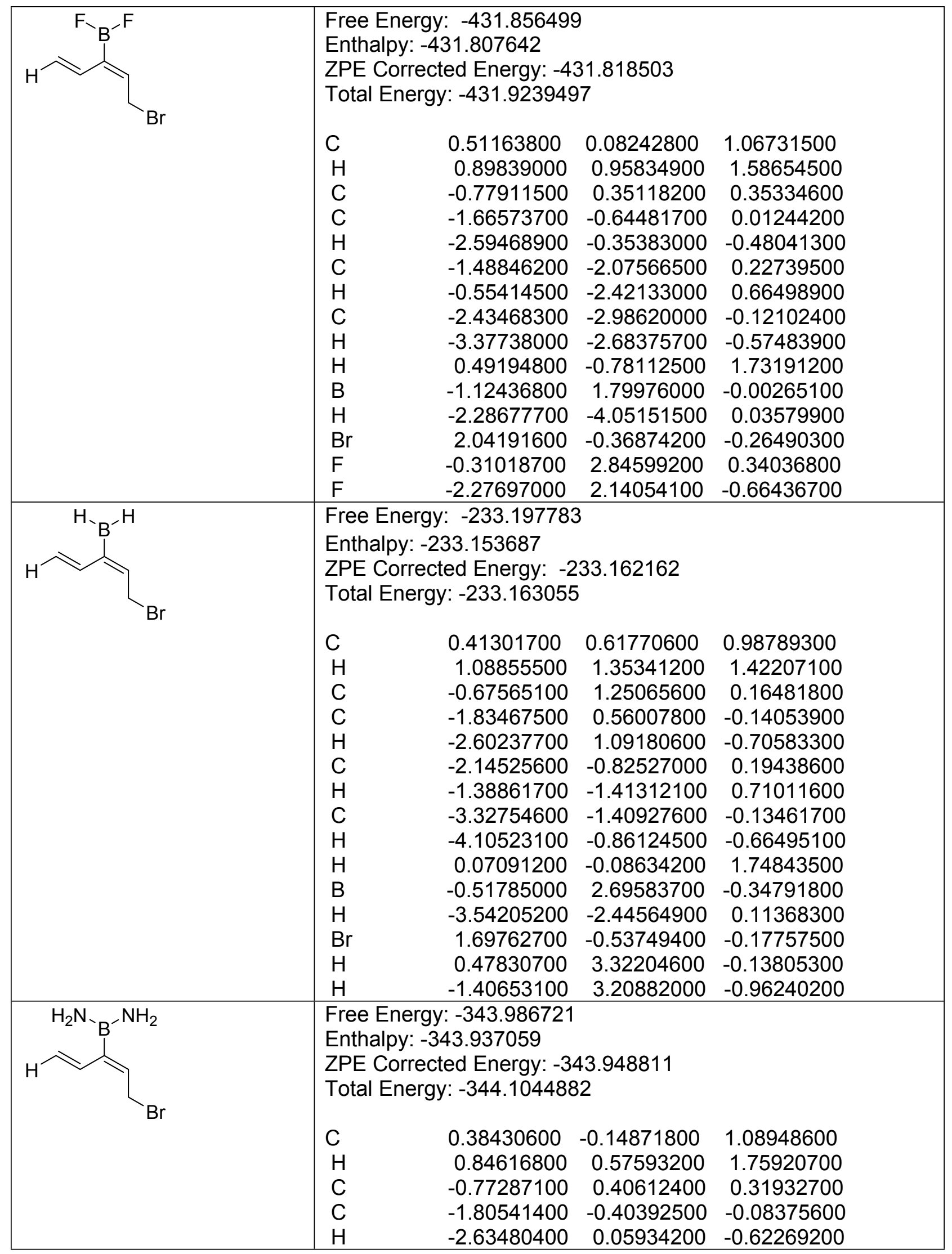




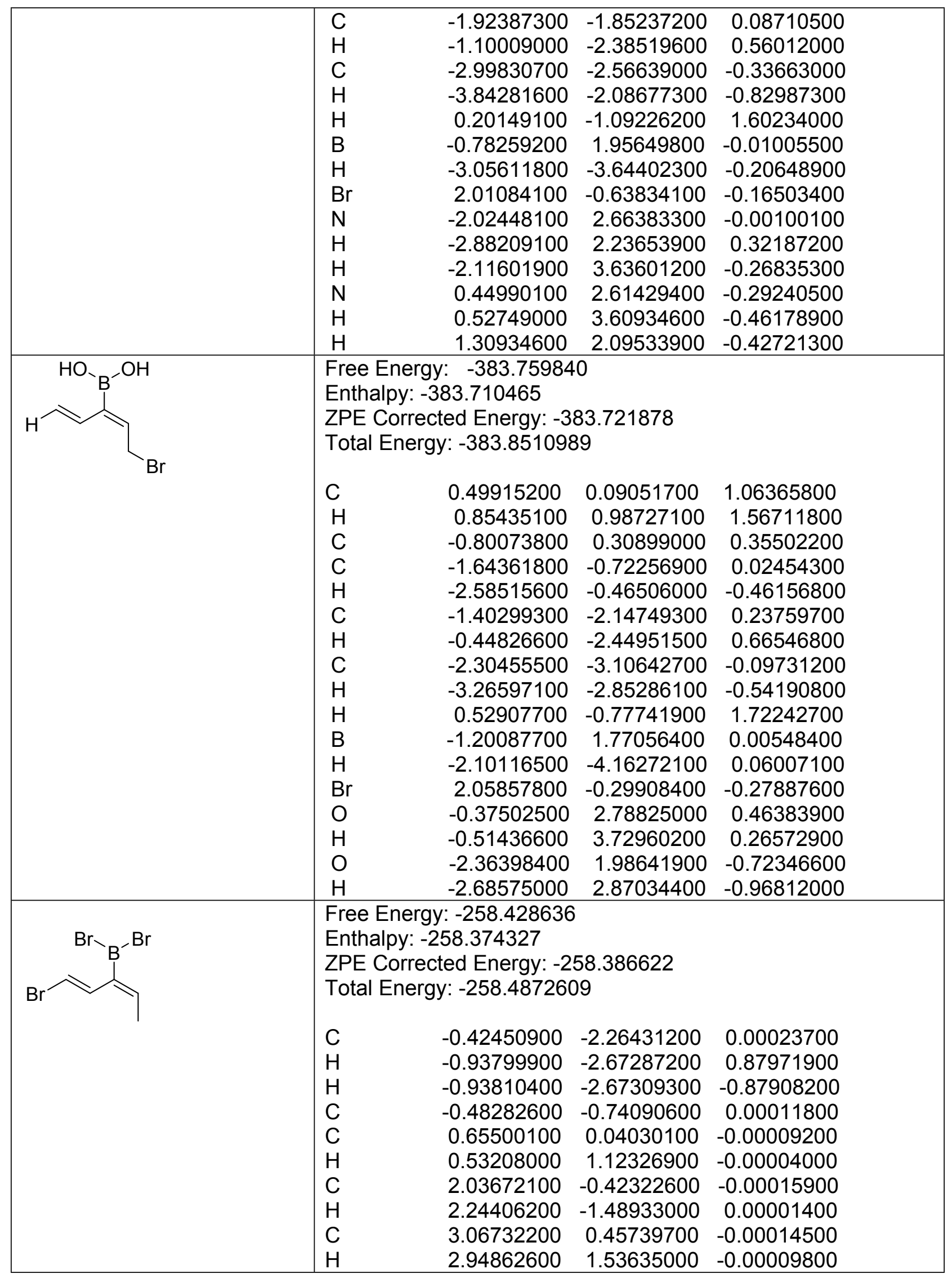




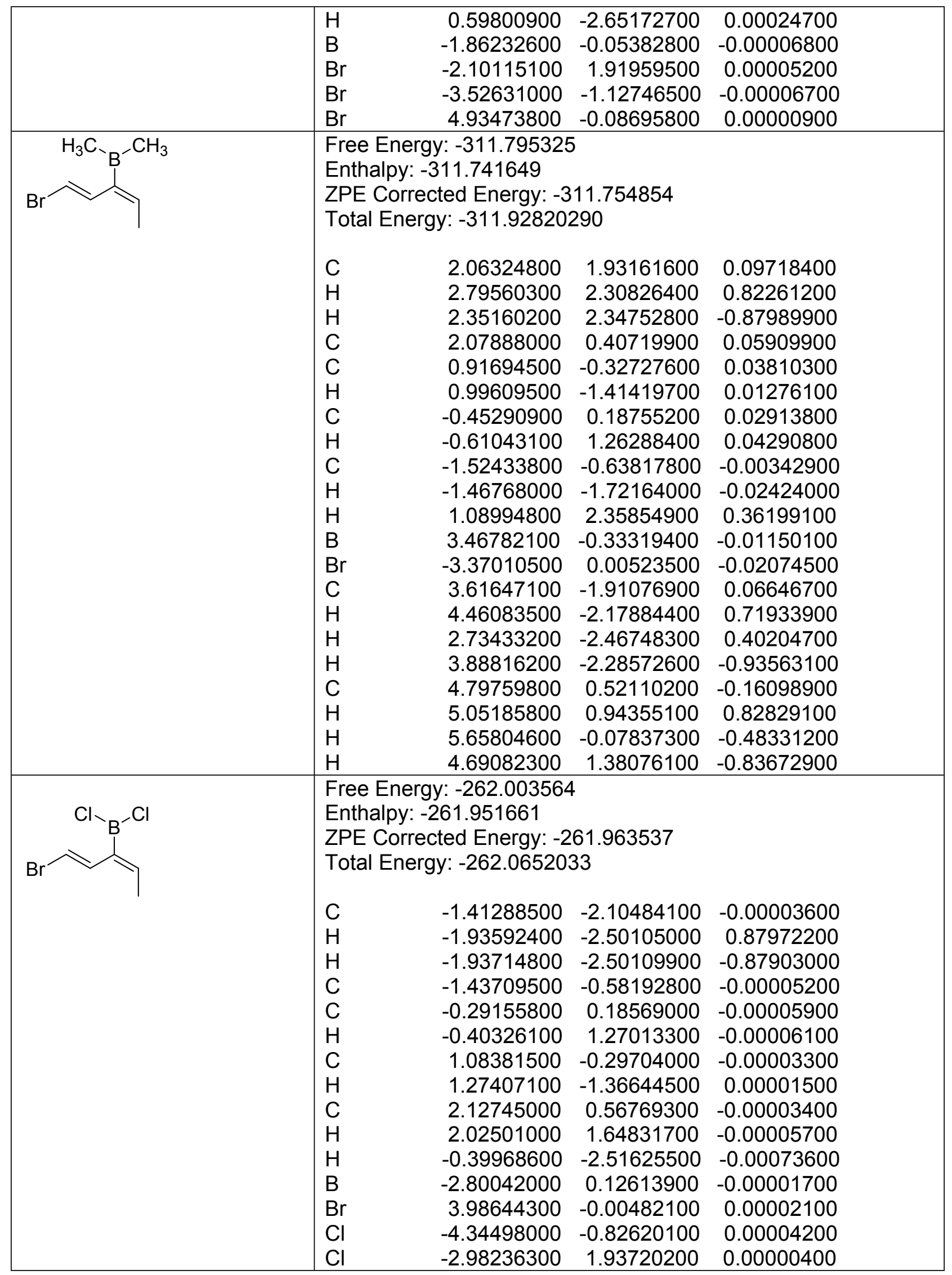




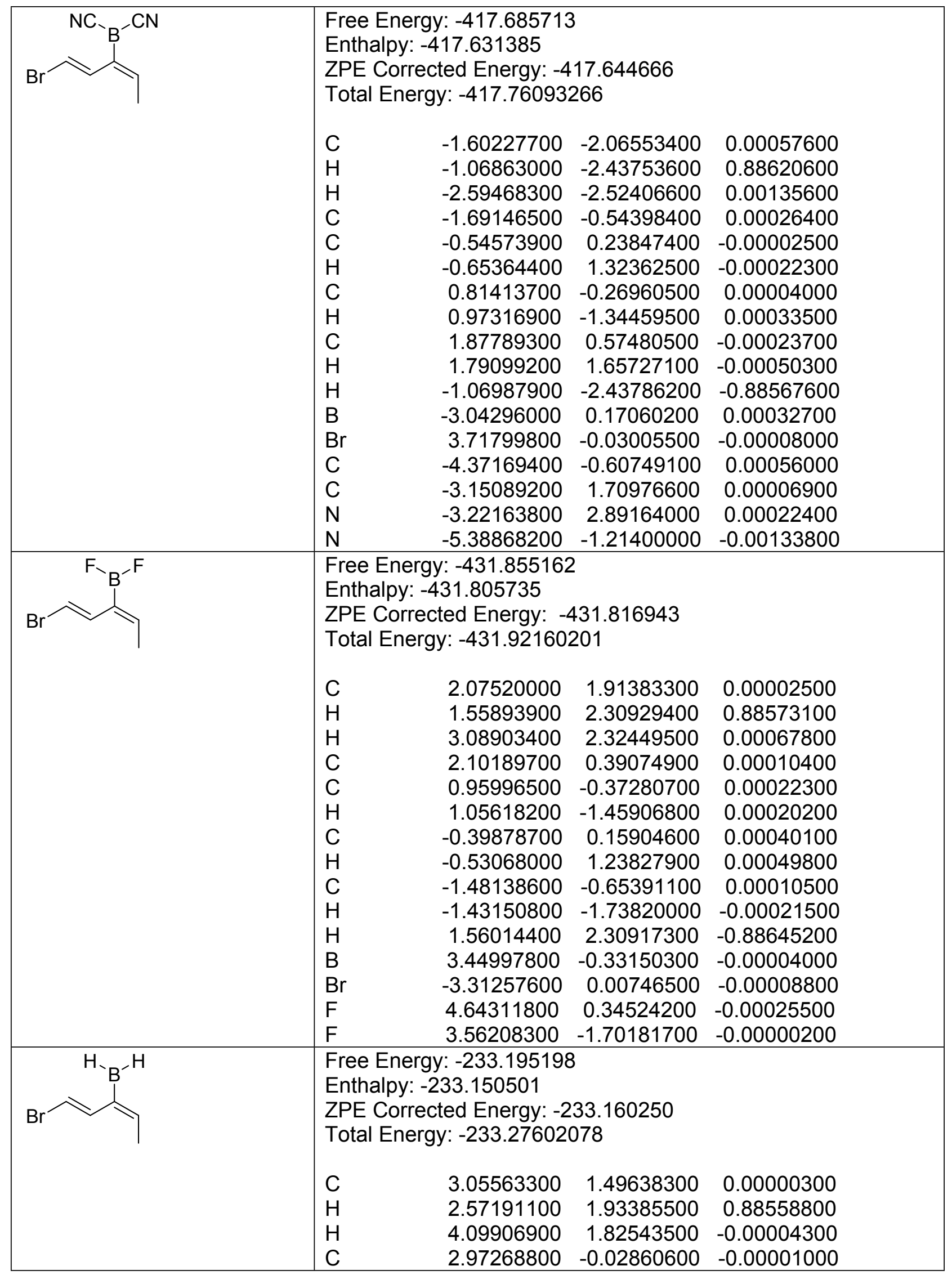




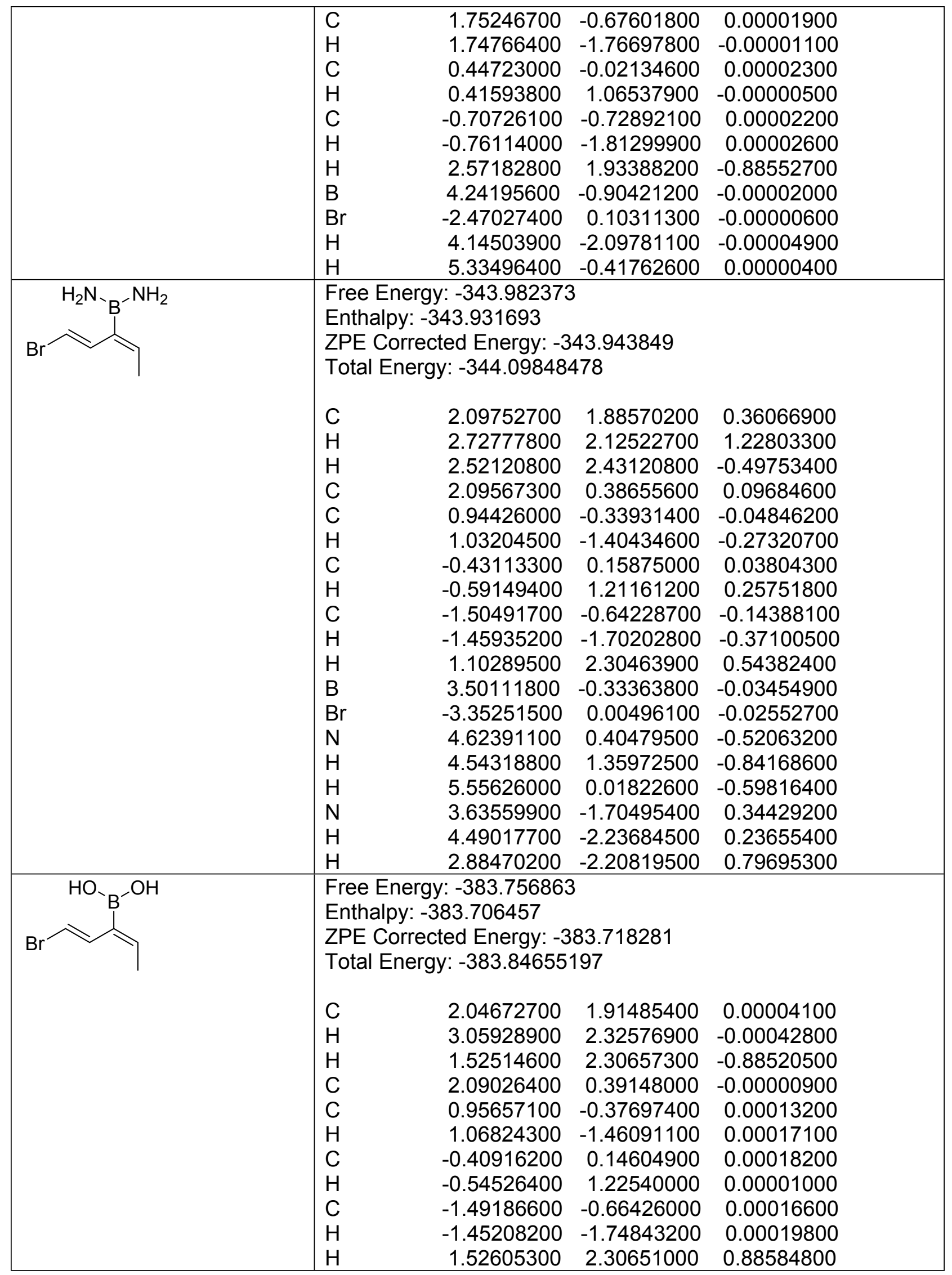




\begin{tabular}{|c|c|c|c|c|}
\hline & $\begin{array}{l}\mathrm{B} \\
\mathrm{Br} \\
\mathrm{O} \\
\mathrm{H} \\
\mathrm{O} \\
\mathrm{H}\end{array}$ & $\begin{array}{r}3.47154200 \\
-3.33047500 \\
4.61601300 \\
5.51655900 \\
3.51107500 \\
4.33904900 \\
\end{array}$ & $\begin{array}{r}-0.32143200 \\
0.00738000 \\
0.46969900 \\
0.10255100 \\
-1.71375400 \\
-2.22305800 \\
\end{array}$ & $\begin{array}{c}-0.00003100 \\
-0.00006000 \\
-0.00012700 \\
0.00008800 \\
-0.00002200 \\
-0.00028700 \\
\end{array}$ \\
\hline$\overbrace{}^{\mathrm{Br}}$ & $\begin{array}{l}\mathrm{C} \\
\mathrm{H} \\
\mathrm{H} \\
\mathrm{C} \\
\mathrm{C} \\
\mathrm{H} \\
\mathrm{C} \\
\mathrm{H} \\
\mathrm{C} \\
\mathrm{H} \\
\mathrm{H} \\
\mathrm{B} \\
\mathrm{N} \\
\mathrm{H} \\
\mathrm{H} \\
\mathrm{Br} \\
\mathrm{Br}\end{array}$ & $\begin{array}{r}0.30364100 \\
-0.22837700 \\
1.36321000 \\
-0.33865300 \\
-1.79556100 \\
-2.14723400 \\
-2.71772600 \\
-3.75251400 \\
-2.52447900 \\
-1.52229200 \\
-2.59055200 \\
0.53305800 \\
-3.51392000 \\
-4.43593800 \\
-3.54180000 \\
2.05772000 \\
0.26297500\end{array}$ & $\begin{array}{l} \\
01.123977 \\
01 \\
1.92906300 \\
2.50080100 \\
2.14441800 \\
0.98599600 \\
0.76836700 \\
1.00080000 \\
0.36799800 \\
0.27462700 \\
0.09711200 \\
0.40621600 \\
-0.98159700 \\
0.07452000 \\
0.77285000 \\
0.34861300 \\
1.78443400 \\
0.79362500 \\
-1.88368500\end{array}$ & $\begin{array}{r} \\
-1.97470300 \\
-2.73546400 \\
-1.86248100 \\
-1.21992500 \\
-1.43702100 \\
-2.44418900 \\
-0.52644000 \\
-0.86447200 \\
0.95929400 \\
1.28278900 \\
1.15674200 \\
-0.31565600 \\
1.81685200 \\
1.84488400 \\
1.72987200 \\
0.72598200 \\
-0.27039900\end{array}$ \\
\hline ( & $\begin{array}{l}\mathrm{C} \\
\mathrm{H} \\
\mathrm{H} \\
\mathrm{C} \\
\mathrm{C} \\
\mathrm{H} \\
\mathrm{C} \\
\mathrm{H} \\
\mathrm{C} \\
\mathrm{H} \\
\mathrm{H} \\
\mathrm{B} \\
\mathrm{N} \\
\mathrm{H} \\
\mathrm{H} \\
\mathrm{Br} \\
\mathrm{Br}\end{array}$ & $\begin{array}{r}0.34802400 \\
-0.17204500 \\
1.40333100 \\
-0.30234700 \\
-1.75165300 \\
-2.08479700 \\
-2.66462000 \\
-3.70048600 \\
-2.43792400 \\
-1.44690900 \\
-2.44178800 \\
0.53385800 \\
-3.44671600 \\
-4.31261100 \\
-3.54794100 \\
2.11249100 \\
0.13356900 \\
\end{array}$ & $\begin{array}{l} \\
00.910847 \\
-2.10485000 \\
-2.76070100 \\
-2.30523000 \\
-1.08531400 \\
-0.87513400 \\
-1.12725800 \\
-0.44325100 \\
-0.34329400 \\
-0.13972200 \\
-0.49236900 \\
0.94458100 \\
-0.07942200 \\
-0.73392400 \\
-0.22531300 \\
-1.73986000 \\
-0.65472200 \\
1.83968300 \\
\end{array}$ & $\begin{array}{r} \\
1.75576000 \\
2.45240000 \\
1.59142900 \\
1.13538200 \\
1.40386200 \\
2.40977000 \\
0.50992000 \\
0.83503500 \\
-0.96125500 \\
-1.27690800 \\
-1.12404200 \\
0.30679700 \\
-1.83828400 \\
-1.95667700 \\
-1.79362600 \\
-0.71186000 \\
0.34427700 \\
\end{array}$ \\
\hline
\end{tabular}




\begin{tabular}{|c|c|c|c|c|}
\hline $\mathrm{Cl}_{\mathrm{B}^{-}}-\mathrm{Cl}$ & $\begin{array}{l}\mathrm{C} \\
\mathrm{H} \\
\mathrm{H} \\
\mathrm{C} \\
\mathrm{C} \\
\mathrm{H} \\
\mathrm{C} \\
\mathrm{H} \\
\mathrm{C} \\
\mathrm{H} \\
\mathrm{H} \\
\mathrm{B} \\
\mathrm{N} \\
\mathrm{H} \\
\mathrm{H} \\
\mathrm{Cl} \\
\mathrm{Cl}\end{array}$ & $\begin{array}{r}1.06456700 \\
0.64504100 \\
2.07747500 \\
0.34726500 \\
-1.03437100 \\
-1.19879900 \\
-2.09456500 \\
-3.04107000 \\
-2.17784100 \\
-1.27942100 \\
-2.21556800 \\
1.08679500 \\
-3.35443600 \\
-4.23828400 \\
-3.42130100 \\
2.40777800 \\
0.77390900\end{array}$ & $\begin{array}{l} \\
04.700638 \\
17 \\
2.39253500 \\
3.36617900 \\
2.39046400 \\
1.24226900 \\
1.33765900 \\
2.17561700 \\
0.54021100 \\
0.76749500 \\
-0.61194500 \\
-0.64912200 \\
-1.56880600 \\
-0.10130400 \\
-0.54517100 \\
-0.81304000 \\
0.29447200 \\
-0.28588800 \\
-154028300\end{array}$ & $\begin{array}{r}-0.13065000 \\
-0.38467900 \\
0.26399500 \\
-0.31409000 \\
-0.86304100 \\
-1.54320600 \\
-0.58191000 \\
-1.07842800 \\
0.41082000 \\
1.03934800 \\
-0.12734000 \\
-0.10744500 \\
1.29538900 \\
0.87341300 \\
1.86302400 \\
1.12753900 \\
-1.16068000\end{array}$ \\
\hline $\mathrm{Cl}_{\mathrm{B}^{-}}$ & $\begin{array}{l}\text { Fre } \\
\text { En } \\
\text { ZP } \\
\text { To } \\
\text { C } \\
\mathrm{H} \\
\mathrm{H} \\
\mathrm{C} \\
\mathrm{C} \\
\mathrm{H} \\
\mathrm{C} \\
\mathrm{H} \\
\mathrm{C} \\
\mathrm{H} \\
\mathrm{H} \\
\mathrm{B} \\
\mathrm{N} \\
\mathrm{H} \\
\mathrm{H} \\
\mathrm{Cl} \\
\mathrm{Cl}\end{array}$ & $\begin{array}{l}:-304.53446 \\
04.485169 \\
\text { ted Energy: }-3 \\
y:-304.62687 \\
1.02895700 \\
0.59956800 \\
2.02679700 \\
0.34148500 \\
-1.02125300 \\
-1.17019700 \\
-2.06400600 \\
-3.01429800 \\
-2.10231800 \\
-1.20188400 \\
-2.09410700 \\
1.07079600 \\
-3.27895400 \\
-4.13170600 \\
-3.40822900 \\
2.42718700 \\
0.68431700\end{array}$ & $\begin{array}{l} \\
04.496768 \\
71 \\
2.37942700 \\
3.34987600 \\
2.38066600 \\
1.23023000 \\
1.29790100 \\
2.07873800 \\
0.50687000 \\
0.66599200 \\
-0.56456000 \\
-0.51073100 \\
-1.55864000 \\
-0.11287700 \\
-0.47510700 \\
-0.90353800 \\
0.39534700 \\
-0.26233800 \\
-1.56747400\end{array}$ & $\begin{array}{r}-0.10705700 \\
-0.35111400 \\
0.32354300 \\
-0.33880800 \\
-0.93580900 \\
-1.68027200 \\
-0.61131700 \\
-1.12163500 \\
0.46483900 \\
1.09056600 \\
0.00260700 \\
-0.11794100 \\
1.32959700 \\
0.99541400 \\
1.82913000 \\
1.05874900 \\
-1.09620800\end{array}$ \\
\hline $\mathrm{NC}_{\mathrm{B}^{-}}-\mathrm{CN}$ & \multicolumn{4}{|c|}{$\begin{array}{l}\text { Free Energy: }-460.420405 \\
\text { Enthalpy: }-460.367733 \\
\text { ZPE Corrected Energy: }-460.381035 \\
\text { Total Energy: }-460.5239053\end{array}$} \\
\hline
\end{tabular}




\begin{tabular}{|c|c|c|c|c|}
\hline & $\begin{array}{l}\mathrm{H} \\
\mathrm{C} \\
\mathrm{C} \\
\mathrm{H} \\
\mathrm{C} \\
\mathrm{H} \\
\mathrm{C} \\
\mathrm{H} \\
\mathrm{H} \\
\mathrm{B} \\
\mathrm{N} \\
\mathrm{H} \\
\mathrm{H} \\
\mathrm{C} \\
\mathrm{C} \\
\mathrm{N} \\
\mathrm{N}\end{array}$ & $\begin{array}{r}2.39850200 \\
0.54510200 \\
-0.84146900 \\
-0.97973700 \\
-1.93913900 \\
-2.88986700 \\
-2.05667400 \\
-1.12487100 \\
-2.18903500 \\
1.18618800 \\
-3.17725300 \\
-4.07624200 \\
-3.22094300 \\
2.43509800 \\
0.69301800 \\
3.39332800 \\
0.32700900\end{array}$ & $\begin{array}{r}2.16311300 \\
1.16695100 \\
1.36847200 \\
2.22706000 \\
0.63512300 \\
0.92937000 \\
-0.51195300 \\
-0.61813300 \\
-1.46166700 \\
-0.22344700 \\
-0.35959100 \\
-0.72312900 \\
0.50834300 \\
-0.39846800 \\
-1.48150000 \\
-0.52708900 \\
-2.44741900\end{array}$ & $\begin{array}{r}0.12787800 \\
-0.35871000 \\
-0.85058000 \\
-1.51066600 \\
-0.53418800 \\
-0.98375100 \\
0.46052600 \\
1.03648400 \\
-0.07481500 \\
-0.14094000 \\
1.39421000 \\
1.10241300 \\
1.91582400 \\
0.74396700 \\
-0.87617700 \\
1.42710900 \\
-1.45435300\end{array}$ \\
\hline NC, & $\begin{array}{l}\mathrm{C} \\
\mathrm{H} \\
\mathrm{H} \\
\mathrm{C} \\
\mathrm{C} \\
\mathrm{H} \\
\mathrm{C} \\
\mathrm{H} \\
\mathrm{C} \\
\mathrm{H} \\
\mathrm{H} \\
\mathrm{B} \\
\mathrm{N} \\
\mathrm{H} \\
\mathrm{H} \\
\mathrm{C} \\
\mathrm{C} \\
\mathrm{N} \\
\mathrm{N}\end{array}$ & $\begin{array}{l}:-460.231617 \\
60.180354 \\
\text { ted Energy: }-4 \\
y:-460.33832 \\
1.40752400 \\
1.10879500 \\
2.41849300 \\
0.55708800 \\
-0.82453600 \\
-0.96443600 \\
-1.90446800 \\
-2.87599400 \\
-1.96295800 \\
-1.00338400 \\
-2.08388000 \\
1.14394700 \\
-3.11537200 \\
-3.46009600 \\
-3.14291300 \\
2.38538700 \\
0.59661200 \\
3.33240700 \\
0.17665000\end{array}$ & $\begin{array}{l}60.193187 \\
71 \\
2.22923400 \\
3.24505900 \\
2.10046400 \\
1.17608200 \\
1.39675100 \\
2.24296500 \\
0.66270100 \\
0.92620100 \\
-0.48236500 \\
-0.56519800 \\
-1.42506500 \\
-0.23320600 \\
-0.31277400 \\
-1.13854900 \\
0.53486500 \\
-0.46084600 \\
-1.45445400 \\
-0.61488300 \\
-2.38053200\end{array}$ & $\begin{array}{r} \\
-0.19535400 \\
-0.45123700 \\
0.18431100 \\
-0.35697100 \\
-0.85048700 \\
-1.52147800 \\
-0.51018200 \\
-0.92395200 \\
0.47499500 \\
1.02620500 \\
-0.07300900 \\
-0.16280100 \\
1.35279900 \\
1.82396000 \\
1.90566500 \\
0.72463800 \\
-0.92639100 \\
1.40443700 \\
-1.51694300\end{array}$ \\
\hline $\mathrm{F} \backslash_{\mathrm{B}^{-}}{ } \mathrm{F}$ & $\begin{array}{l}\text { Free Energy: }-474.593214 \\
\text { Enthalpy: }-474.545635 \\
\text { ZPE Corrected Energy: }-474.556698 \\
\text { Total Energy: }-474.6884257\end{array}$ & $\begin{array}{l}y:-474.593214 \\
74.545635 \\
\text { ted Energy: }-4 \\
y:-474.68842 \\
-1.97380100 \\
-1.82901700 \\
-2.92728700 \\
-1.00523600 \\
0.26805200\end{array}$ & $\begin{array}{l} \\
74.556698 \\
57 \\
1.68246100 \\
2.76309000 \\
1.31963800 \\
0.83271400 \\
1.38368500 \\
\end{array}$ & $\begin{array}{r}-0.43882700 \\
-0.43995600 \\
-0.81543100 \\
0.01667100 \\
0.54773400\end{array}$ \\
\hline
\end{tabular}




\begin{tabular}{|c|c|c|c|c|}
\hline & $\begin{array}{l}\mathrm{H} \\
\mathrm{C} \\
\mathrm{H} \\
\mathrm{C} \\
\mathrm{H} \\
\mathrm{H} \\
\mathrm{B} \\
\mathrm{N} \\
\mathrm{H} \\
\mathrm{H} \\
\mathrm{F} \\
\mathrm{F}\end{array}$ & $\begin{array}{r}0.18148500 \\
1.50035400 \\
2.31200000 \\
1.95498200 \\
1.21047600 \\
2.04510500 \\
-1.37784500 \\
3.24861100 \\
4.06835200 \\
3.27513900 \\
-2.49849900 \\
-0.68521500\end{array}$ & $\begin{array}{r}2.38727300 \\
0.81510900 \\
1.38804200 \\
-0.50334900 \\
-0.88104900 \\
-1.27021500 \\
-0.66231600 \\
-0.41110700 \\
-0.35556700 \\
0.24514800 \\
-1.14904300 \\
-1.59215100\end{array}$ & $\begin{array}{r}0.96983500 \\
0.55034400 \\
1.00625100 \\
-0.06570100 \\
-0.77496600 \\
0.71487900 \\
0.08194800 \\
-0.76548400 \\
-0.16857700 \\
-1.54040000 \\
-0.54683900 \\
0.80635900\end{array}$ \\
\hline $\mathrm{B}^{\mathrm{F}} \mathrm{B}^{-\mathrm{F}}$ & $\begin{array}{l}\mathrm{C} \\
\mathrm{H} \\
\mathrm{H} \\
\mathrm{C} \\
\mathrm{C} \\
\mathrm{H} \\
\mathrm{C} \\
\mathrm{H} \\
\mathrm{C} \\
\mathrm{H} \\
\mathrm{H} \\
\mathrm{B} \\
\mathrm{N} \\
\mathrm{H} \\
\mathrm{H} \\
\mathrm{F} \\
\mathrm{F}\end{array}$ & $\begin{array}{r}1.92704000 \\
1.78499000 \\
2.85390900 \\
0.98915100 \\
-0.25835600 \\
-0.15695100 \\
-1.47526700 \\
-2.30274800 \\
-1.87051900 \\
-1.11342400 \\
-1.90025400 \\
1.34076900 \\
-3.17058800 \\
-3.99092500 \\
-3.27017900 \\
2.43068500 \\
0.64860000\end{array}$ & $\begin{array}{l} \\
74.371820 \\
49 \\
1.66501000 \\
2.74446300 \\
1.28345200 \\
0.83789600 \\
1.38239500 \\
2.34997600 \\
0.80028300 \\
1.31229200 \\
-0.48371100 \\
-0.75340200 \\
-1.31370500 \\
-0.65761800 \\
-0.40261100 \\
-0.54289400 \\
0.30995700 \\
-1.16748200 \\
-1.55418500\end{array}$ & $\begin{array}{r}0.50576000 \\
0.52533100 \\
0.92614300 \\
-0.02111500 \\
-0.61504700 \\
-1.10523500 \\
-0.58830700 \\
-1.08102100 \\
0.12367800 \\
0.86973900 \\
-0.59025600 \\
-0.09862300 \\
0.78989400 \\
0.21522600 \\
1.50177800 \\
0.55111800 \\
-0.85419100\end{array}$ \\
\hline $\mathrm{H}_{2} \mathrm{~N}$ & $\begin{array}{l}\mathrm{C} \\
\mathrm{H} \\
\mathrm{H} \\
\mathrm{C} \\
\mathrm{C} \\
\mathrm{H} \\
\mathrm{C} \\
\mathrm{H} \\
\mathrm{C} \\
\mathrm{H} \\
\mathrm{H}\end{array}$ & $\begin{array}{r}-2.76476700 \\
-3.04402400 \\
-3.53178400 \\
-1.52641700 \\
-0.51346800 \\
-0.90536200 \\
0.82841400 \\
1.42574300 \\
1.65554200 \\
1.06458100 \\
1.93692600 \\
\end{array}$ & $\begin{array}{l} \\
75.901089 \\
-0.36438600 \\
-1.41846100 \\
0.35432500 \\
0.03900000 \\
-0.99533900 \\
-2.00993000 \\
-0.83057000 \\
-1.71310800 \\
0.42970900 \\
1.19302100 \\
0.87225400 \\
\end{array}$ & $\begin{array}{r}0.46018900 \\
0.52258100 \\
0.74211700 \\
0.02701200 \\
-0.29431600 \\
-0.41057700 \\
-0.42268200 \\
-0.66659300 \\
-0.20252600 \\
0.31889000 \\
-1.16853100 \\
\end{array}$ \\
\hline
\end{tabular}




\begin{tabular}{|c|c|c|c|c|}
\hline & $\begin{array}{l}\mathrm{B} \\
\mathrm{H} \\
\mathrm{H} \\
\mathrm{N} \\
\mathrm{H} \\
\mathrm{H}\end{array}$ & $\begin{array}{r}-1.38873400 \\
-2.08766900 \\
-0.65970300 \\
2.89537400 \\
3.64532000 \\
2.75619500\end{array}$ & $\begin{array}{r}1.55496900 \\
2.33918500 \\
1.95693300 \\
0.20319300 \\
-0.25404800 \\
-0.18784900\end{array}$ & $\begin{array}{r}-0.27888800 \\
0.29361700 \\
-1.13386500 \\
0.56375700 \\
0.05305900 \\
1.49138000\end{array}$ \\
\hline $\mathrm{H}_{-\mathrm{B}^{-}} \mathrm{H}^{-{ }^{-}}$ & $\begin{array}{l}\mathrm{C} \\
\mathrm{H} \\
\mathrm{H} \\
\mathrm{C} \\
\mathrm{C} \\
\mathrm{H} \\
\mathrm{C} \\
\mathrm{H} \\
\mathrm{C} \\
\mathrm{H} \\
\mathrm{H} \\
\mathrm{B} \\
\mathrm{H} \\
\mathrm{H} \\
\mathrm{N} \\
\mathrm{H} \\
\mathrm{H}\end{array}$ & $\begin{array}{r}-2.73002500 \\
-3.03105500 \\
-3.46539900 \\
-1.50578100 \\
-0.52282700 \\
-0.92808300 \\
0.81180400 \\
1.43031500 \\
1.59701200 \\
0.98945600 \\
1.82951000 \\
-1.31436200 \\
-1.94279300 \\
-0.61322700 \\
2.85588300 \\
3.63581700 \\
2.77499400\end{array}$ & $\begin{array}{l} \\
75.766151 \\
67 \\
-0.31908900 \\
-1.36248300 \\
0.42832900 \\
0.03628800 \\
-1.02082900 \\
-2.02357400 \\
-0.84777800 \\
-1.70904600 \\
0.42631100 \\
1.12946700 \\
0.93634500 \\
1.53667200 \\
2.36409800 \\
1.86885100 \\
0.20075100 \\
-0.13367600 \\
-0.23634800\end{array}$ & $\begin{array}{r}0.49995000 \\
0.60166300 \\
0.78852600 \\
0.01962500 \\
-0.31738300 \\
-0.46241700 \\
-0.42635800 \\
-0.68271500 \\
-0.15807500 \\
0.42691800 \\
-1.10005000 \\
-0.33247100 \\
0.25498300 \\
-1.23663800 \\
0.55636700 \\
0.00443100 \\
1.46653500\end{array}$ \\
\hline (N) & $\begin{array}{l}\mathrm{C} \\
\mathrm{H} \\
\mathrm{H} \\
\mathrm{C} \\
\mathrm{C} \\
\mathrm{H} \\
\mathrm{C} \\
\mathrm{H} \\
\mathrm{C} \\
\mathrm{H} \\
\mathrm{H} \\
\mathrm{B} \\
\mathrm{N} \\
\mathrm{H} \\
\mathrm{H} \\
\mathrm{N}\end{array}$ & $\begin{array}{r}-2.02179100 \\
-1.89003000 \\
-3.00353100 \\
-1.02120400 \\
0.27137400 \\
0.21550200 \\
1.50080800 \\
2.32633100 \\
1.93468700 \\
1.12191700 \\
2.19624000 \\
-1.33059000 \\
3.11158200 \\
3.97992900 \\
2.95207100 \\
-1.88142000 \\
\end{array}$ & $\begin{array}{l} \\
86.688023 \\
1.70896400 \\
2.79211800 \\
1.33691000 \\
0.87264700 \\
1.48241900 \\
2.55218400 \\
0.90701800 \\
1.54862500 \\
-0.50745100 \\
-1.05906800 \\
-1.06890500 \\
-0.68025400 \\
-0.54662200 \\
-0.23138000 \\
-0.10290000 \\
-1.36383800 \\
\end{array}$ & $\begin{array}{r}-0.42066200 \\
-0.45887100 \\
-0.70832600 \\
-0.01978200 \\
0.38240800 \\
0.60728100 \\
0.47797700 \\
0.80064200 \\
0.11538900 \\
-0.36193000 \\
1.02387200 \\
0.09908200 \\
-0.78378500 \\
-0.35628700 \\
-1.68578300 \\
-1.02616500\end{array}$ \\
\hline
\end{tabular}




\begin{tabular}{|c|c|c|c|c|}
\hline & $\begin{array}{l}\mathrm{H} \\
\mathrm{H} \\
\mathrm{N} \\
\mathrm{H} \\
\mathrm{H}\end{array}$ & $\begin{array}{l}-2.00749800 \\
-2.16551600 \\
-1.09400500 \\
-0.72463600 \\
-1.28417300\end{array}$ & $\begin{array}{l}-0.89130900 \\
-2.33566300 \\
-1.34126900 \\
-0.83804400 \\
-2.32077900\end{array}$ & $\begin{array}{r}-1.91133600 \\
-1.02013500 \\
1.34080900 \\
2.13662900 \\
1.51084400\end{array}$ \\
\hline 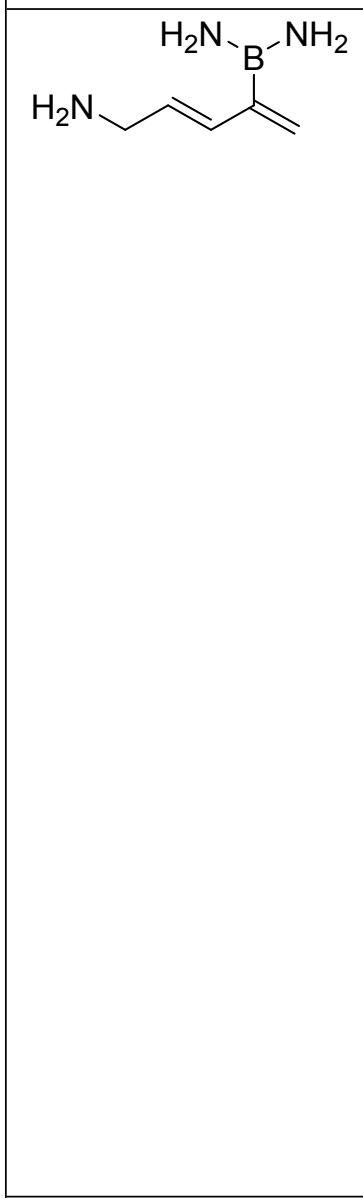 & $\begin{array}{l}\mathrm{C} \\
\mathrm{H} \\
\mathrm{H} \\
\mathrm{C} \\
\mathrm{C} \\
\mathrm{H} \\
\mathrm{C} \\
\mathrm{H} \\
\mathrm{C} \\
\mathrm{H} \\
\mathrm{H} \\
\mathrm{B} \\
\mathrm{N} \\
\mathrm{H} \\
\mathrm{H} \\
\mathrm{N} \\
\mathrm{H} \\
\mathrm{H} \\
\mathrm{N} \\
\mathrm{H} \\
\mathrm{H}\end{array}$ & $\begin{array}{r}-1.98271000 \\
-1.84647000 \\
-2.94602200 \\
-1.01373900 \\
0.25944700 \\
0.19507300 \\
1.47149600 \\
2.31552900 \\
1.84742800 \\
1.05404400 \\
1.95429300 \\
-1.31236600 \\
3.10326300 \\
3.94914100 \\
3.08906400 \\
-1.97335300 \\
-2.16918000 \\
-2.26352800 \\
-0.93971200 \\
-0.49331000 \\
-1.09972000\end{array}$ & $\begin{array}{l} \\
86.518728 \\
78 \\
1.70188600 \\
2.78289000 \\
1.32537900 \\
0.87243400 \\
1.46178700 \\
2.49285700 \\
0.86240800 \\
1.42919600 \\
-0.50622600 \\
-0.89563600 \\
-1.22909500 \\
-0.67629700 \\
-0.49626600 \\
-0.38151800 \\
0.07636800 \\
-1.34798900 \\
-0.87186200 \\
-2.31610000 \\
-1.33425000 \\
-0.82064500 \\
-2.31454500\end{array}$ & $\begin{array}{r}-0.46744600 \\
-0.50411600 \\
-0.80460600 \\
-0.00984900 \\
0.47904400 \\
0.83204400 \\
0.50791900 \\
0.90646800 \\
-0.03957200 \\
-0.68297600 \\
0.77781200 \\
0.12336500 \\
-0.80422800 \\
-0.25685900 \\
-1.64132400 \\
-0.94276300 \\
-1.81273800 \\
-0.91060600 \\
1.32797200 \\
2.07668700 \\
1.51594000\end{array}$ \\
\hline (NO & $\begin{array}{l}\text { Fre } \\
\text { En } \\
\text { ZP } \\
\text { To } \\
\text { C } \\
\text { H } \\
\text { H } \\
\text { C } \\
\text { C } \\
\text { H } \\
\text { C } \\
\text { H } \\
\text { C } \\
\text { H } \\
\text { H } \\
\text { B }\end{array}$ & $\begin{array}{l}\mathrm{y}:-426.493748 \\
426.445345 \\
\text { cted Energy: }-4 \\
y \mathrm{y}:-426.612440 \\
-1.94646500 \\
-1.77811600 \\
-2.90947200 \\
-0.99177600 \\
0.30144100 \\
0.24115000 \\
1.52855000 \\
2.35519100 \\
1.95356400 \\
1.19161300 \\
2.04232700 \\
-1.39265400\end{array}$ & $\begin{array}{l} \\
26.456925 \\
06 \\
1.74427100 \\
2.82220800 \\
1.40243500 \\
0.86330600 \\
1.39716000 \\
2.41174100 \\
0.81783600 \\
1.39185600 \\
-0.52165600 \\
-0.90318300 \\
-1.26722800 \\
-0.64704600 \\
\end{array}$ & $\begin{array}{r}-0.38668100 \\
-0.37178400 \\
-0.75662700 \\
0.02947000 \\
0.52894900 \\
0.93234200 \\
0.52013700 \\
0.94894600 \\
-0.06519100 \\
-0.75151600 \\
0.73590000 \\
0.08587800 \\
\end{array}$ \\
\hline
\end{tabular}




\begin{tabular}{|c|c|c|c|c|}
\hline & $\begin{array}{l}\mathrm{N} \\
\mathrm{H} \\
\mathrm{H} \\
\mathrm{O} \\
\mathrm{H} \\
\mathrm{O} \\
\mathrm{H}\end{array}$ & $\begin{array}{r}3.24208500 \\
4.05772700 \\
-2.24766600 \\
-2.74558400 \\
-0.73945300 \\
-0.97332500 \\
\end{array}$ & $\begin{array}{c}-0.47564800 \\
-0.34741200 \\
0.16005800 \\
-1.07105000 \\
-1.97679700 \\
-1.49198400 \\
-2.43013900 \\
\end{array}$ & $\begin{array}{r}-0.79090700 \\
-0.19616600 \\
-1.58552200 \\
-0.72113700 \\
-0.69690600 \\
0.97217400 \\
1.07989600 \\
\end{array}$ \\
\hline - & $\begin{array}{l}\mathrm{C} \\
\mathrm{H} \\
\mathrm{H} \\
\mathrm{C} \\
\mathrm{C} \\
\mathrm{H} \\
\mathrm{C} \\
\mathrm{H} \\
\mathrm{C} \\
\mathrm{H} \\
\mathrm{H} \\
\mathrm{B} \\
\mathrm{N} \\
\mathrm{H} \\
\mathrm{H} \\
\mathrm{Br} \\
\mathrm{Br}\end{array}$ & $\begin{array}{l}y:-301.140695 \\
301.091561 \\
\text { ted Energy: }-30 \\
y:-301.226893 \\
-1148.5861 \\
-1.49385500 \\
-2.09841700 \\
-1.02795600 \\
-0.80113600 \\
-1.63314200 \\
-1.16254600 \\
-2.99049800 \\
-3.51807100 \\
-3.73896800 \\
-4.13624300 \\
-2.71652100 \\
0.65889900 \\
-4.69719500 \\
-5.45140500 \\
-4.49485400 \\
1.67656000 \\
1.69879600\end{array}$ & $\begin{array}{l} \\
01.102536 \\
35 \\
1.23858400 \\
1.24793900 \\
2.20031900 \\
0.02812100 \\
-1.14956800 \\
-2.12687100 \\
-1.05718700 \\
-1.94033900 \\
0.21686800 \\
0.60901800 \\
1.06024100 \\
0.01404300 \\
0.30438400 \\
0.97434500 \\
-0.09978100 \\
-1.68578600 \\
1.69188000\end{array}$ & $\begin{array}{c}0.87831800 \\
1.78875500 \\
0.65981100 \\
0.54577000 \\
0.43503300 \\
0.36009300 \\
0.18371900 \\
-0.17481600 \\
0.11404300 \\
1.05591800 \\
0.09157400 \\
0.14259400 \\
-0.91722900 \\
-0.86715000 \\
-1.82157400 \\
-0.08539500 \\
-0.15249900\end{array}$ \\
\hline$\underbrace{\mathrm{Br}}_{\mathrm{NH}_{2}}$ & $\begin{array}{l}\text { MC } \\
\text { Fre } \\
\text { En } \\
\text { ZP } \\
\text { To } \\
\text { Fre } \\
\text { C } \\
\mathrm{H} \\
\mathrm{H} \\
\mathrm{C} \\
\mathrm{C} \\
\mathrm{H} \\
\mathrm{C} \\
\mathrm{H} \\
\mathrm{C} \\
\mathrm{H} \\
\mathrm{H} \\
\mathrm{B} \\
\mathrm{N}\end{array}$ & $\begin{array}{l}\text { NL2DZ } \\
\text { y: }-300.921070 \\
300.872374 \\
\text { ted Energy: }-3 \\
y:-301.009259 \\
-1160.2483 \\
\\
1.48016200 \\
2.10581900 \\
1.01001900 \\
0.78990700 \\
1.61548000 \\
1.14638600 \\
2.96626600 \\
3.49916200 \\
3.70565400 \\
4.08212900 \\
2.67218400 \\
-0.66388900 \\
4.66442100 \\
\end{array}$ & $\begin{array}{l}\text { l } \\
00.883200 \\
99 \\
-1.23699200 \\
-1.24681700 \\
-2.19631300 \\
-0.03078200 \\
1.14399800 \\
2.12100900 \\
1.04290900 \\
1.92122100 \\
-0.22741800 \\
-0.65317700 \\
-1.06994900 \\
-0.01416100 \\
-0.29461600 \\
\end{array}$ & $\begin{array}{r}0.86980300 \\
1.76390800 \\
0.65470200 \\
0.55524600 \\
0.45015100 \\
0.37742300 \\
0.19304300 \\
-0.16400200 \\
0.10707700 \\
1.03924300 \\
0.05344400 \\
0.14453400 \\
-0.91689600 \\
\end{array}$ \\
\hline
\end{tabular}




\begin{tabular}{|c|c|c|c|c|}
\hline & $\begin{array}{l}\mathrm{H} \\
\mathrm{H} \\
\mathrm{Br} \\
\mathrm{Br}\end{array}$ & $\begin{array}{r}5.42651500 \\
4.47159900 \\
-1.65797800 \\
-1.68745500\end{array}$ & $\begin{array}{r}-0.95357100 \\
0.14118500 \\
1.67946200 \\
-1.68177000\end{array}$ & $\begin{array}{l}-0.87385200 \\
-1.80713700 \\
-0.08748100 \\
-0.15252000\end{array}$ \\
\hline$\underbrace{\mathrm{C}}_{\mathrm{NH}_{2}}$ & $\begin{array}{l}\mathrm{C} \\
\mathrm{H} \\
\mathrm{H} \\
\mathrm{C} \\
\mathrm{C} \\
\mathrm{H} \\
\mathrm{C} \\
\mathrm{H} \\
\mathrm{C} \\
\mathrm{H} \\
\mathrm{H} \\
\mathrm{B} \\
\mathrm{N} \\
\mathrm{H} \\
\mathrm{H} \\
\mathrm{Cl} \\
\mathrm{Cl}\end{array}$ & $\begin{array}{c}-0.70735300 \\
-1.28800800 \\
-0.23813700 \\
-0.02923200 \\
-0.85791900 \\
-0.38426500 \\
-2.22310700 \\
-2.75742100 \\
-2.97551000 \\
-3.33454500 \\
-1.95449600 \\
1.41687000 \\
-3.97518800 \\
-4.72677300 \\
-3.80951900 \\
2.33403300 \\
2.37148800\end{array}$ & $\begin{array}{l} \\
04.678931 \\
1 \\
1.27865800 \\
1.33737200 \\
2.22446400 \\
0.05144200 \\
-1.13036500 \\
-2.10767700 \\
-1.05041700 \\
-1.94702200 \\
0.21851500 \\
0.65254500 \\
1.06274600 \\
0.01821300 \\
0.26207000 \\
0.93478400 \\
-0.18068300 \\
-1.54888700 \\
1.54247000\end{array}$ & $\begin{array}{c}0.77547600 \\
1.69942400 \\
0.50133600 \\
0.48192300 \\
0.44223600 \\
0.38819600 \\
0.22774100 \\
-0.08430900 \\
0.13257700 \\
1.07134500 \\
0.03458000 \\
0.04224000 \\
-0.86191500 \\
-0.81032200 \\
-1.75553800 \\
-0.15995000 \\
-0.28606300\end{array}$ \\
\hline$\underbrace{\mathrm{Cl}}_{\mathrm{NH}_{2}}$ & $\begin{array}{l}\text { MOt } \\
\text { Fre } \\
\text { Ent } \\
\text { ZPE } \\
\text { Tot } \\
\text { Fre } \\
\text { C } \\
\mathrm{H} \\
\mathrm{H} \\
\mathrm{C} \\
\mathrm{C} \\
\mathrm{H} \\
\mathrm{C} \\
\mathrm{H} \\
\mathrm{C} \\
\mathrm{H} \\
\mathrm{H} \\
\mathrm{B} \\
\mathrm{N} \\
\mathrm{H} \\
\mathrm{H} \\
\mathrm{Cl} \\
\mathrm{Cl}\end{array}$ & $\begin{array}{l}\text { VL2DZ } \\
\text { y: }-304.505036 \\
04.458827 \\
\text { ted Energy: }-3 \\
y:-304.596252 \\
-1202.7414 \\
\\
-0.69836300 \\
-1.29943400 \\
-0.22278600 \\
-0.02539000 \\
-0.84860600 \\
-0.37726100 \\
-2.20804100 \\
-2.74982800 \\
-2.95060900 \\
-3.28490500 \\
-1.91888500 \\
1.41473900 \\
-3.95468200 \\
-4.71287600 \\
-3.80720000 \\
2.30730000 \\
2.36142400\end{array}$ & $\begin{array}{l} \\
\\
\\
4.469227 \\
-1.28222700 \\
-1.35037300 \\
-2.22166400 \\
-0.05650000 \\
1.12145900 \\
2.09929900 \\
1.03341300 \\
1.92715500 \\
-0.23102800 \\
-0.70756200 \\
-1.06993800 \\
-0.01632600 \\
-0.24760700 \\
-0.91096000 \\
0.24108500 \\
1.54561700 \\
-1.52813800\end{array}$ & $\begin{array}{r}-0.75181300 \\
-1.65999100 \\
-0.47085100 \\
-0.48527200 \\
-0.46184900 \\
-0.41713500 \\
-0.24433300 \\
0.05651500 \\
-0.12463700 \\
-1.04830700 \\
0.01544300 \\
-0.03977300 \\
0.85767900 \\
0.81560900 \\
1.72881500 \\
0.16557300 \\
0.28045300\end{array}$ \\
\hline
\end{tabular}




\begin{tabular}{|c|c|c|c|c|}
\hline (NC) & $\begin{array}{l}\mathrm{C} \\
\mathrm{H} \\
\mathrm{H} \\
\mathrm{C} \\
\mathrm{C} \\
\mathrm{H} \\
\mathrm{C} \\
\mathrm{H} \\
\mathrm{C} \\
\mathrm{H} \\
\mathrm{H} \\
\mathrm{B} \\
\mathrm{N} \\
\mathrm{H} \\
\mathrm{H} \\
\mathrm{C} \\
\mathrm{C} \\
\mathrm{N} \\
\mathrm{N}\end{array}$ & $\begin{array}{r}-0.50759000 \\
-1.08333100 \\
-0.05933600 \\
0.16045000 \\
-0.67169100 \\
-0.19771800 \\
-2.03028100 \\
-2.58464500 \\
-2.77374200 \\
-3.18596300 \\
-1.78163500 \\
1.60293500 \\
-3.71949100 \\
-4.47878400 \\
-3.51711100 \\
2.38840000 \\
2.36357300 \\
2.97374500 \\
2.93133600\end{array}$ & $\begin{array}{l}60.364669 \\
68 \\
\\
1.26630000 \\
1.31398700 \\
2.21961100 \\
0.04314300 \\
-1.14546000 \\
-2.12399700 \\
-1.06030300 \\
-1.95637000 \\
0.22415800 \\
0.62518500 \\
1.05797000 \\
0.01985700 \\
0.28378700 \\
0.94988300 \\
-0.15726600 \\
1.32289500 \\
-1.31022300 \\
2.33121300 \\
-2.33947900\end{array}$ & $\begin{array}{c}0.80594000 \\
1.73333000 \\
0.52093400 \\
0.47613500 \\
0.44646200 \\
0.41048000 \\
0.23529700 \\
-0.03905700 \\
0.12426600 \\
1.05683200 \\
0.06123200 \\
0.03548100 \\
-0.91669800 \\
-0.89128900 \\
-1.80402100 \\
-0.22438600 \\
-0.15040100 \\
-0.43243600 \\
-0.29454000\end{array}$ \\
\hline NC: & $\begin{array}{l}\text { MC } \\
\text { Fre } \\
\text { En } \\
\text { ZP } \\
\text { To } \\
\text { Fre } \\
\text { C } \\
\text { H } \\
\text { H } \\
\text { C } \\
\text { C } \\
\text { H } \\
\text { C } \\
\text { H } \\
\text { C } \\
\text { H } \\
\text { H } \\
\text { B } \\
\text { N } \\
\mathrm{H} \\
\mathrm{H} \\
\text { C } \\
\text { C } \\
\mathrm{N} \\
\mathrm{N}\end{array}$ & $\begin{array}{r}-0.49965800 \\
-1.09748400 \\
-0.04449200 \\
0.16707700 \\
-0.65959700 \\
-0.18700100 \\
-2.01081800 \\
-2.57258000 \\
-2.74209000 \\
-3.13781700 \\
-1.73996900 \\
1.59626100 \\
-3.68430900 \\
-4.44909500 \\
-3.48666400 \\
2.36694200 \\
2.34944300 \\
2.92203800 \\
2.89170000\end{array}$ & $\begin{array}{l}l \\
60.169685 \\
31 \\
\\
-1.25590100 \\
-1.30068600 \\
-2.20655700 \\
-0.03847600 \\
1.14764100 \\
2.12583300 \\
1.05090100 \\
1.93887000 \\
-0.23233200 \\
-0.66123600 \\
-1.06324100 \\
-0.01897700 \\
-0.27625300 \\
-0.93376100 \\
0.19111700 \\
-1.33274500 \\
1.31660500 \\
-2.34916900 \\
2.34976300\end{array}$ & $\begin{array}{r}-0.80802900 \\
-1.71982300 \\
-0.52796000 \\
-0.49149800 \\
-0.46127400 \\
-0.42551400 \\
-0.24208700 \\
0.03636800 \\
-0.11693100 \\
-1.04062800 \\
-0.03010800 \\
-0.03908200 \\
0.92020700 \\
0.90342400 \\
1.79363000 \\
0.22748200 \\
0.15764000 \\
0.43338100 \\
0.30558400\end{array}$ \\
\hline
\end{tabular}




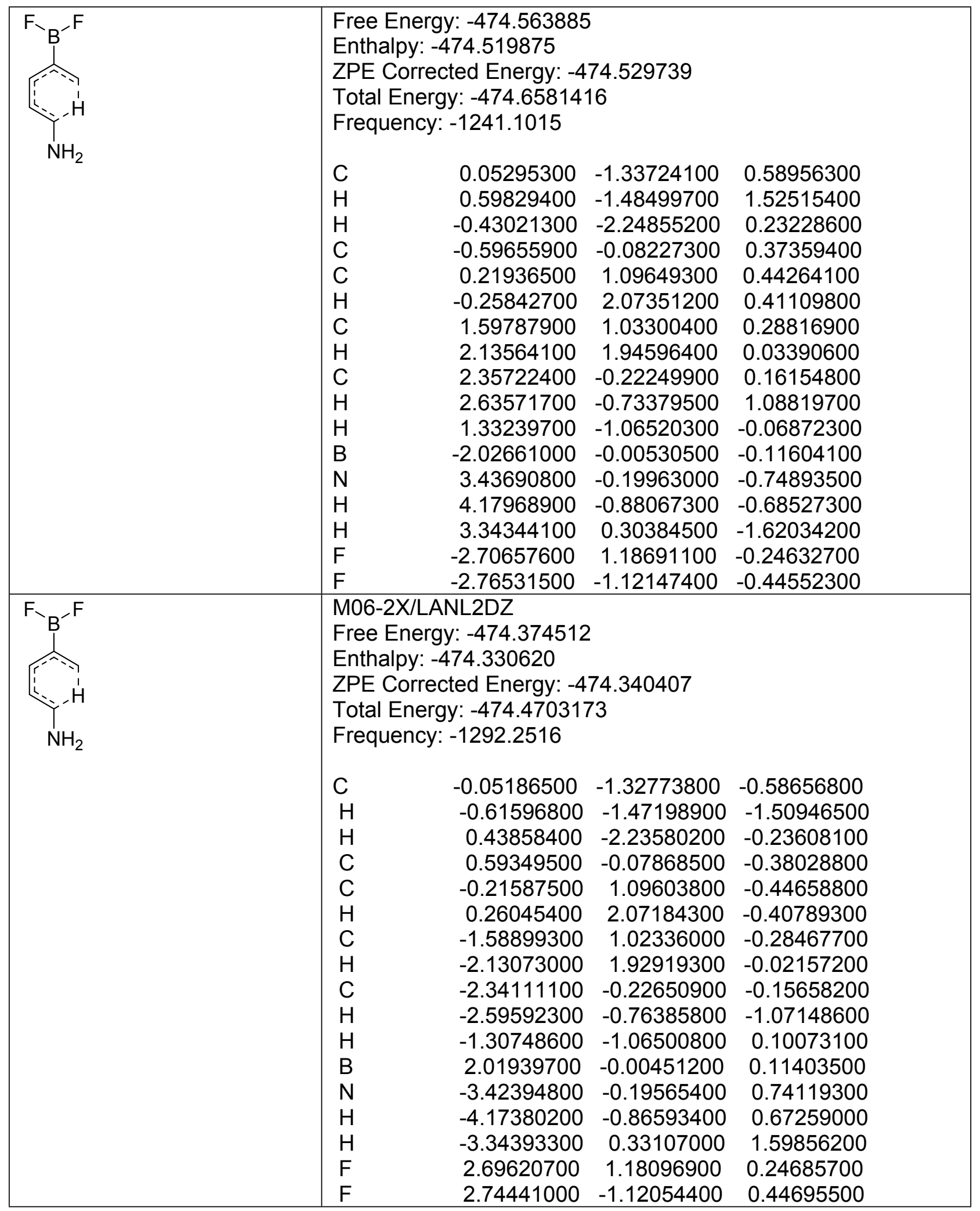




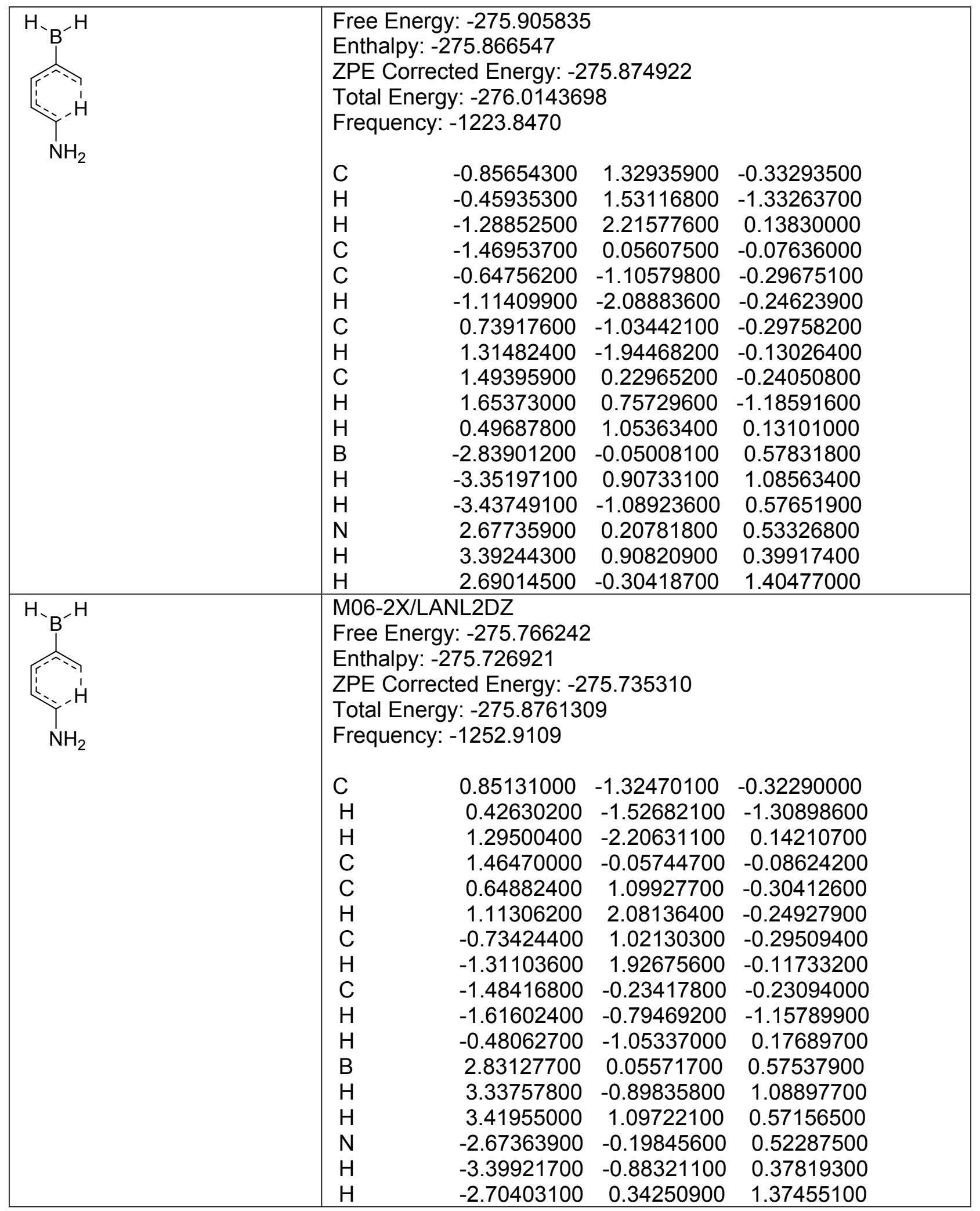




\begin{tabular}{|c|c|c|c|c|}
\hline 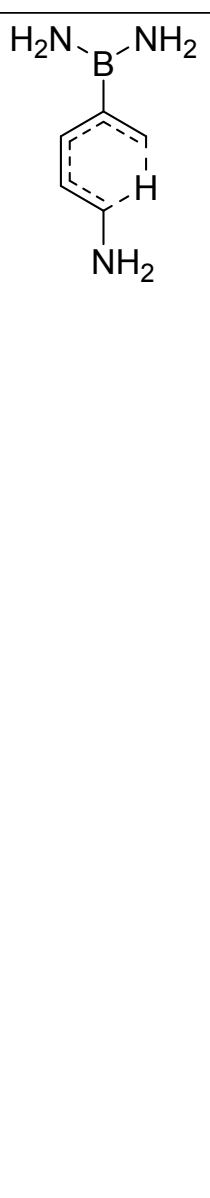 & $\begin{array}{l}\mathrm{C} \\
\mathrm{H} \\
\mathrm{H} \\
\mathrm{C} \\
\mathrm{C} \\
\mathrm{H} \\
\mathrm{C} \\
\mathrm{H} \\
\mathrm{C} \\
\mathrm{H} \\
\mathrm{H} \\
\mathrm{B} \\
\mathrm{N} \\
\mathrm{H} \\
\mathrm{H} \\
\mathrm{N} \\
\mathrm{H} \\
\mathrm{H} \\
\mathrm{N} \\
\mathrm{H} \\
\mathrm{H}\end{array}$ & $\begin{array}{r}-0.09807900 \\
-0.65482500 \\
0.40090300 \\
0.58429900 \\
-0.21517700 \\
0.27119400 \\
-1.60565600 \\
-2.09455000 \\
-2.40261100 \\
-2.62849700 \\
-1.36395300 \\
2.08176200 \\
-3.55474600 \\
-4.27433300 \\
-3.47458500 \\
2.63430900 \\
3.60276500 \\
2.07170400 \\
2.88722900 \\
3.84674500 \\
2.54442300\end{array}$ & $\begin{array}{l} \\
86.649511 \\
13 \\
-1.18071000 \\
-1.10501300 \\
-2.15067100 \\
-0.01712400 \\
1.13729000 \\
2.05939000 \\
1.07084000 \\
1.88063400 \\
-0.13053100 \\
-0.41265300 \\
-1.05124800 \\
-0.05767300 \\
-0.27728500 \\
-0.93697900 \\
-0.06111300 \\
-1.25844000 \\
-1.35321200 \\
-2.08228800 \\
1.12001900 \\
1.18221400 \\
1.95065800\end{array}$ & $\begin{array}{r}-0.86400200 \\
-1.80273900 \\
-0.77835000 \\
-0.38558700 \\
-0.14445800 \\
0.17378700 \\
0.01440600 \\
0.55858600 \\
-0.20246500 \\
-1.23463500 \\
-0.16122800 \\
0.09946100 \\
0.62435900 \\
0.36528700 \\
1.60866000 \\
0.64969100 \\
0.92772700 \\
0.81204500 \\
-0.02720200 \\
0.28856400 \\
-0.49031000\end{array}$ \\
\hline 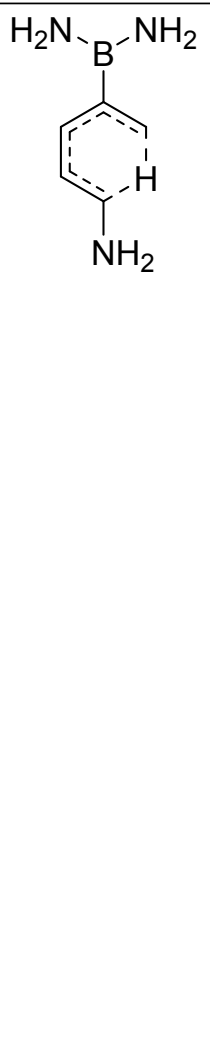 & $\begin{array}{l}\text { MC } \\
\text { Fre } \\
\text { En } \\
\text { ZP } \\
\text { To } \\
\text { Fre } \\
\text { C } \\
\text { H } \\
\text { H } \\
\text { C } \\
\text { C } \\
\text { H } \\
\text { C } \\
\mathrm{H} \\
\text { C } \\
\mathrm{H} \\
\mathrm{H} \\
\mathrm{B} \\
\mathrm{N} \\
\mathrm{H} \\
\mathrm{H} \\
\mathrm{N} \\
\mathrm{H}\end{array}$ & $\begin{array}{l}\text { NL2DZ } \\
y:-386.510147 \\
386.464691 \\
\text { ted Energy: }-3 \\
y:-386.65568 \\
-1407.6203 \\
\\
0.09333600 \\
0.67181600 \\
-0.41464100 \\
-0.58205000 \\
0.20788100 \\
-0.28074400 \\
1.59316400 \\
2.08493400 \\
2.38535500 \\
2.58894100 \\
1.34149200 \\
-2.07340000 \\
3.53935000 \\
4.27507600 \\
3.47674600 \\
-2.59707300 \\
-3.56270000\end{array}$ & $\begin{array}{l}\text { l } \\
86.475517 \\
98 \\
-1.16054500 \\
-1.07048900 \\
-2.12523000 \\
-0.00821900 \\
1.13922400 \\
2.05024000 \\
1.06097900 \\
1.85367900 \\
-0.12885700 \\
-0.42120500 \\
-1.05549700 \\
-0.06088300 \\
-0.27831900 \\
-0.90990400 \\
-0.04465300 \\
-1.26057600 \\
-1.37378800\end{array}$ & $\begin{array}{r} \\
0.88126500 \\
1.80301100 \\
0.81739500 \\
0.39212000 \\
0.12990200 \\
-0.21074000 \\
-0.03367500 \\
-0.59647200 \\
0.20255400 \\
1.23327500 \\
0.15026400 \\
-0.09901500 \\
-0.61079900 \\
-0.33570400 \\
-1.59060700 \\
-0.66724000 \\
-0.94411300\end{array}$ \\
\hline
\end{tabular}




\begin{tabular}{|c|c|c|c|c|}
\hline & $\begin{array}{l}\mathrm{H} \\
\mathrm{N} \\
\mathrm{H} \\
\mathrm{H}\end{array}$ & $\begin{array}{l}-2.01048800 \\
-2.88554500 \\
-3.83824700 \\
-2.54842400\end{array}$ & $\begin{array}{r}-2.06367900 \\
1.10412000 \\
1.16986900 \\
1.92300400\end{array}$ & $\begin{array}{r}-0.84544800 \\
0.04138400 \\
-0.29036500 \\
0.52817100\end{array}$ \\
\hline (-) & $\begin{array}{l}\mathrm{C} \\
\mathrm{H} \\
\mathrm{H} \\
\mathrm{C} \\
\mathrm{C} \\
\mathrm{H} \\
\mathrm{C} \\
\mathrm{H} \\
\mathrm{C} \\
\mathrm{H} \\
\mathrm{H} \\
\mathrm{B} \\
\mathrm{N} \\
\mathrm{H} \\
\mathrm{H} \\
\mathrm{O} \\
\mathrm{H} \\
\mathrm{O} \\
\mathrm{H}\end{array}$ & $\begin{array}{r}0.06966100 \\
0.62514600 \\
-0.44540700 \\
-0.58220900 \\
0.22880200 \\
-0.26005500 \\
1.61253000 \\
2.13028100 \\
2.37968800 \\
2.62613600 \\
1.33320300 \\
-2.04324300 \\
3.49923100 \\
4.21438000 \\
3.41170900 \\
-2.64641500 \\
-3.57994000 \\
-2.72189500 \\
-3.63821300\end{array}$ & $\begin{array}{r}-1.35072900 \\
-1.52057200 \\
-2.24596700 \\
-0.09142300 \\
1.08218300 \\
2.05276900 \\
1.02887000 \\
1.94148300 \\
-0.21326600 \\
-0.73983000 \\
-1.07038000 \\
-0.00409100 \\
-0.18050500 \\
-0.89039100 \\
0.29565900 \\
1.25337300 \\
1.36852900 \\
-1.18497300 \\
-1.18832000\end{array}$ & $\begin{array}{r}0.56412900 \\
1.49115000 \\
0.21113900 \\
0.38138500 \\
0.43342500 \\
0.38499600 \\
0.26146200 \\
-0.03604000 \\
0.15380100 \\
1.08052200 \\
-0.09408300 \\
-0.09848700 \\
-0.72052500 \\
-0.65313900 \\
-1.60786100 \\
-0.19211800 \\
-0.43884900 \\
-0.41158100 \\
-0.73735000\end{array}$ \\
\hline HO & $\begin{array}{l}\mathrm{C} \\
\mathrm{H} \\
\mathrm{H} \\
\mathrm{C} \\
\mathrm{C} \\
\mathrm{H} \\
\mathrm{C} \\
\mathrm{H} \\
\mathrm{C} \\
\mathrm{H} \\
\mathrm{H} \\
\mathrm{B} \\
\mathrm{N} \\
\mathrm{H} \\
\mathrm{H}\end{array}$ & $\begin{array}{r}-0.06540800 \\
-0.64156500 \\
0.46270200 \\
0.57841900 \\
-0.22361700 \\
0.26856700 \\
-1.60195600 \\
-2.12107100 \\
-2.36289300 \\
-2.58372100 \\
-1.30886500 \\
2.03501200 \\
-3.48694400 \\
-4.21264300 \\
-3.41720100\end{array}$ & $\begin{array}{l} \\
26.244409 \\
8 \\
-1.34544000 \\
-1.51769000 \\
-2.23309600 \\
-0.08993000 \\
1.08073200 \\
2.04771800 \\
1.02028300 \\
1.92534600 \\
-0.21484800 \\
-0.76512500 \\
-1.06991500 \\
-0.00313100 \\
-0.17724000 \\
-0.87248800 \\
0.32271400 \\
\end{array}$ & $\begin{array}{r}-0.56053300 \\
-1.47200100 \\
-0.21222500 \\
-0.39047200 \\
-0.43675300 \\
-0.37869400 \\
-0.25324000 \\
0.05799800 \\
-0.14925300 \\
-1.06482000 \\
0.12537900 \\
0.09500700 \\
0.71006400 \\
0.63222700 \\
1.58401800 \\
\end{array}$ \\
\hline
\end{tabular}




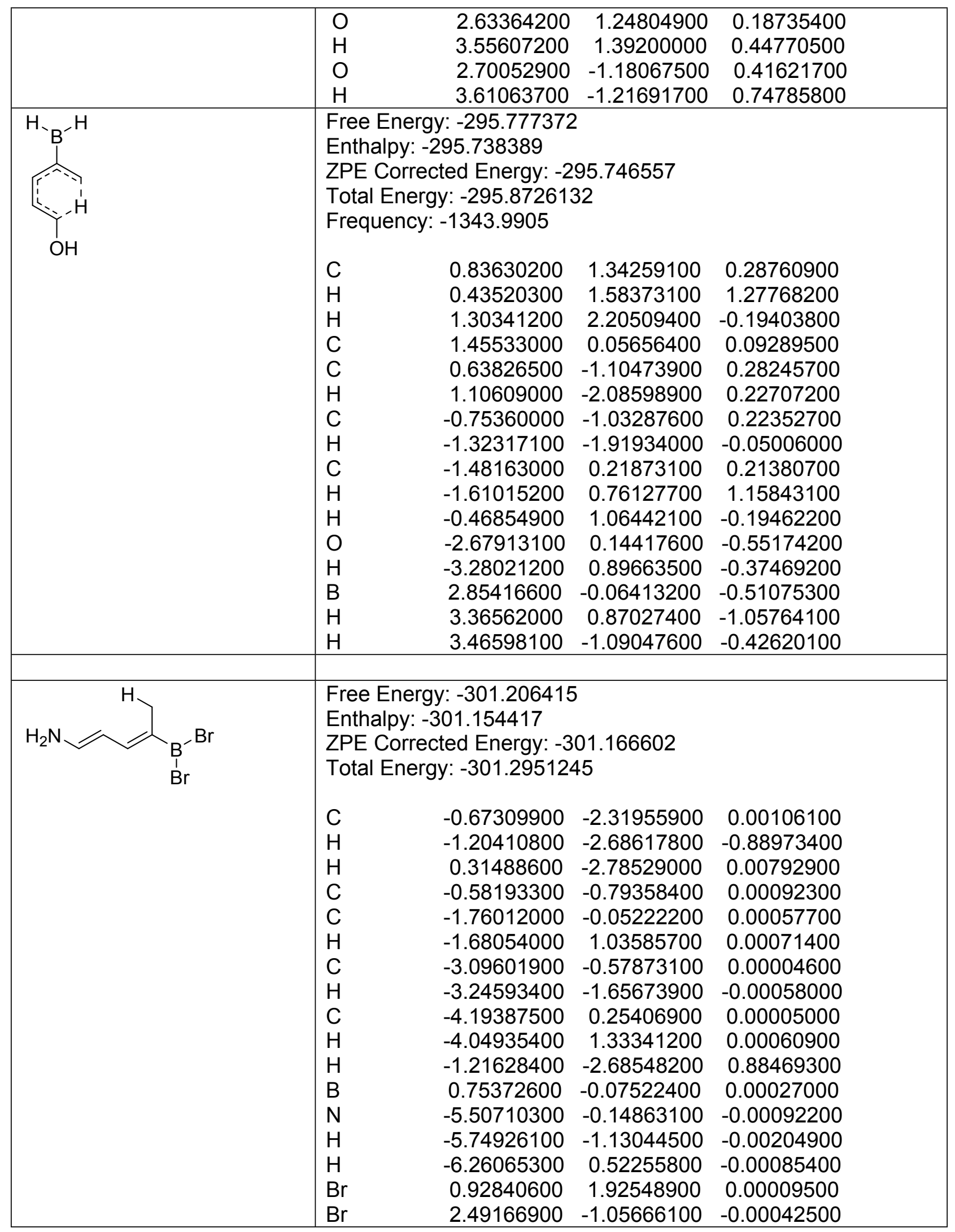




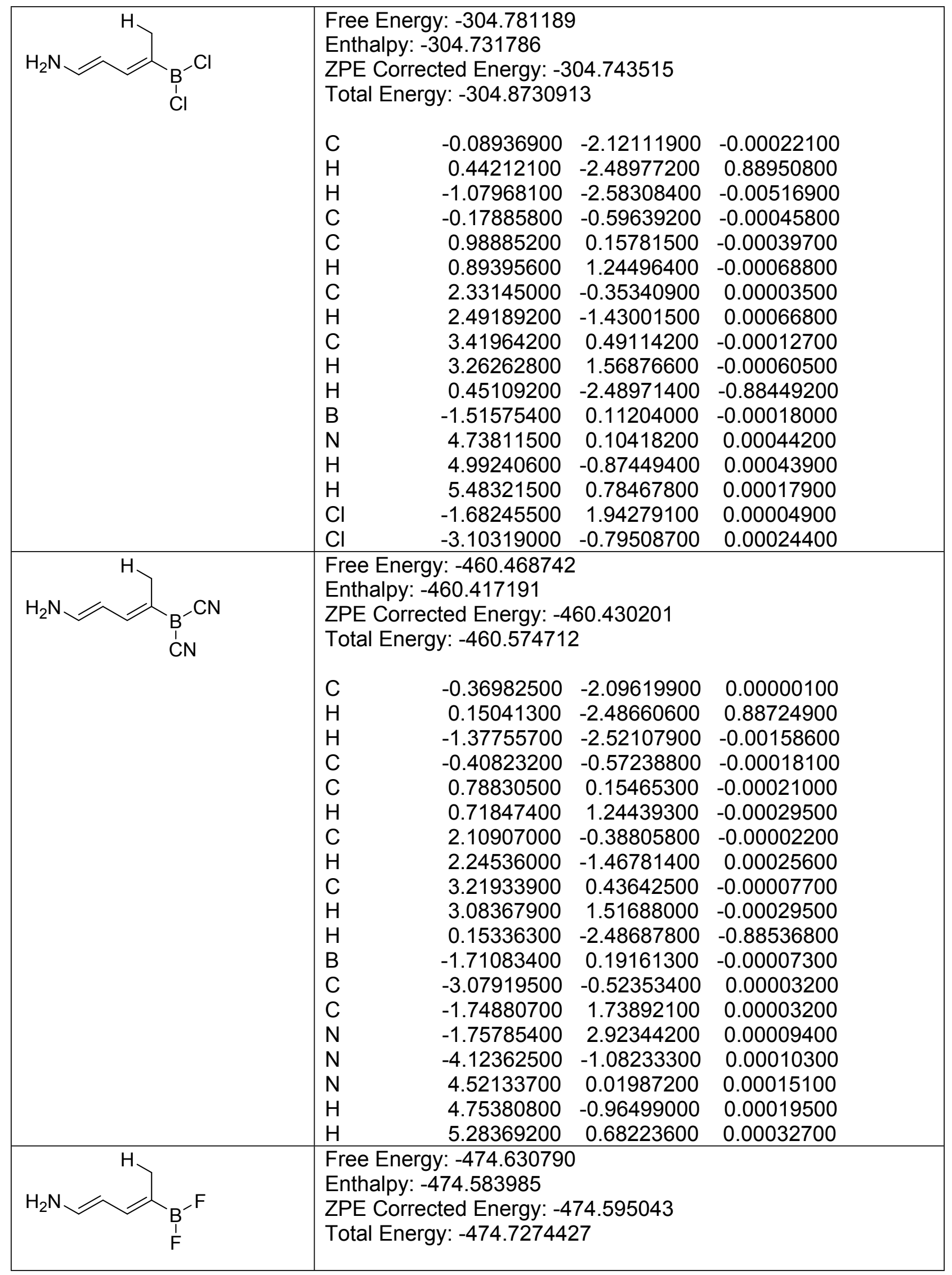




\begin{tabular}{|c|c|c|c|c|}
\hline & $\begin{array}{l}\mathrm{C} \\
\mathrm{H} \\
\mathrm{H} \\
\mathrm{C} \\
\mathrm{C} \\
\mathrm{H} \\
\mathrm{C} \\
\mathrm{H} \\
\mathrm{C} \\
\mathrm{H} \\
\mathrm{H} \\
\mathrm{B} \\
\mathrm{N} \\
\mathrm{H} \\
\mathrm{H} \\
\mathrm{F} \\
\mathrm{F}\end{array}$ & $\begin{array}{r}0.82085000 \\
0.31640400 \\
1.84658500 \\
0.80444300 \\
-0.37934900 \\
-0.31686800 \\
-1.70800900 \\
-1.82237300 \\
-2.82845700 \\
-2.71776800 \\
0.31424400 \\
2.11094700 \\
-4.13293900 \\
-4.34597100 \\
-4.90501800 \\
2.18657300 \\
3.34117500\end{array}$ & $\begin{array}{r}1.92848200 \\
2.33981100 \\
2.31039900 \\
0.40464200 \\
-0.30869000 \\
-1.39932300 \\
0.24918500 \\
1.33226700 \\
-0.54702600 \\
-1.63052900 \\
2.33995600 \\
-0.35819400 \\
-0.10528600 \\
0.88289600 \\
-0.75447700 \\
-1.73969300 \\
0.26718300\end{array}$ & $\begin{array}{r}-0.00002400 \\
0.88693500 \\
-0.00121600 \\
-0.00007000 \\
-0.00011200 \\
-0.00020100 \\
-0.00002900 \\
0.00009200 \\
-0.00003600 \\
-0.00011500 \\
-0.88566900 \\
0.00000100 \\
0.00010600 \\
0.00017100 \\
-0.00004100 \\
0.00004400 \\
0.00005800\end{array}$ \\
\hline $\begin{array}{l}\mathrm{B}^{-}-\mathrm{H} \\
\mathrm{H} \\
\mathrm{H}\end{array}$ & $\begin{array}{l}\mathrm{C} \\
\mathrm{H} \\
\mathrm{H} \\
\mathrm{C} \\
\mathrm{C} \\
\mathrm{H} \\
\mathrm{C} \\
\mathrm{H} \\
\mathrm{C} \\
\mathrm{H} \\
\mathrm{H} \\
\mathrm{B} \\
\mathrm{N} \\
\mathrm{H} \\
\mathrm{H} \\
\mathrm{H} \\
\mathrm{H}\end{array}$ & $\begin{array}{l}y:-275.969831 \\
275.927639 \\
\text { ted Energy: }-2 \\
\text { y: } 276.080749 \\
1.90415900 \\
1.46325000 \\
2.97374500 \\
1.68258000 \\
0.38938700 \\
0.28130200 \\
-0.83713600 \\
-0.78150400 \\
-2.06915400 \\
-2.12984100 \\
1.45783900 \\
2.84397100 \\
-3.28918300 \\
-3.34365400 \\
-4.15357200 \\
2.63186000 \\
3.98597700\end{array}$ & $\begin{array}{l} \\
75.937227 \\
98 \\
1.42355100 \\
1.90366000 \\
1.65741200 \\
-0.08836600 \\
-0.59907500 \\
-1.68677100 \\
0.15895200 \\
1.24681300 \\
-0.45119500 \\
-1.53868100 \\
1.90492500 \\
-1.07647300 \\
0.19003000 \\
1.19933200 \\
-0.32975400 \\
-2.25856900 \\
-0.70941300\end{array}$ & $\begin{array}{r}0.00000200 \\
0.88763200 \\
-0.00301900 \\
-0.00014000 \\
-0.00017700 \\
-0.00010600 \\
-0.00025700 \\
-0.00017400 \\
-0.00024400 \\
-0.00013900 \\
-0.88419000 \\
0.00023800 \\
0.00046200 \\
0.00002300 \\
-0.00029600 \\
0.00044200 \\
0.00029100\end{array}$ \\
\hline $\begin{array}{l}\mathrm{B}^{-}-\mathrm{NH}_{2} \\
\mathrm{~N}_{2}\end{array}$ & $\begin{array}{l}\mathrm{C} \\
\mathrm{H} \\
\mathrm{H} \\
\mathrm{C} \\
\mathrm{C} \\
\mathrm{H} \\
\mathrm{C}\end{array}$ & $\begin{array}{l}\text { y: }-386.748982 \\
386.699535 \\
\text { ted Energy: }-3 \\
\text { y: }-386.89331 \\
0.83292100 \\
1.22559400 \\
1.50052400 \\
0.78084100 \\
-0.40842400 \\
-0.36224600 \\
-1.76454200\end{array}$ & $\begin{array}{l} \\
86.711989 \\
02 \\
1.91429400 \\
2.41234100 \\
2.18643200 \\
0.40106500 \\
-0.27352500 \\
-1.35391800 \\
0.25755400 \\
\end{array}$ & $\begin{array}{r} \\
-0.26322600 \\
0.63923300 \\
-1.09329200 \\
-0.07793800 \\
0.03086900 \\
0.20133700 \\
-0.02410600\end{array}$ \\
\hline
\end{tabular}




\begin{tabular}{|c|c|c|c|c|}
\hline & $\begin{array}{l}\mathrm{H} \\
\mathrm{C} \\
\mathrm{H} \\
\mathrm{H} \\
\mathrm{B} \\
\mathrm{N} \\
\mathrm{H} \\
\mathrm{H} \\
\mathrm{N} \\
\mathrm{H} \\
\mathrm{H} \\
\mathrm{N} \\
\mathrm{H} \\
\mathrm{H}\end{array}$ & $\begin{array}{c}-1.90655700 \\
-2.87006900 \\
-2.75108100 \\
-0.14674200 \\
2.14947300 \\
-4.19419800 \\
-4.95419000 \\
-4.41955700 \\
3.33674400 \\
3.31424900 \\
4.25597500 \\
2.22451800 \\
1.43797100 \\
3.06489200\end{array}$ & $\begin{array}{r}1.32630700 \\
-0.53438600 \\
-1.60448900 \\
2.35975300 \\
-0.37150400 \\
-0.10937700 \\
-0.75961100 \\
0.86379600 \\
0.34231900 \\
1.31799000 \\
-0.07930700 \\
-1.77470000 \\
-2.28257100 \\
-2.32690600 \\
\end{array}$ & $\begin{array}{r}-0.18378000 \\
0.11957800 \\
0.28588500 \\
-0.46838100 \\
0.02010200 \\
0.07646300 \\
0.19803300 \\
-0.07097300 \\
0.39069000 \\
0.65290500 \\
0.42959600 \\
-0.26734200 \\
-0.64760200 \\
-0.15321100 \\
\end{array}$ \\
\hline $\begin{array}{l}\mathrm{B}_{1}^{-} \\
\mathrm{OH}\end{array}$ & $\begin{array}{l}\mathrm{C} \\
\mathrm{H} \\
\mathrm{H} \\
\mathrm{C} \\
\mathrm{C} \\
\mathrm{H} \\
\mathrm{C} \\
\mathrm{H} \\
\mathrm{C} \\
\mathrm{H} \\
\mathrm{H} \\
\mathrm{B} \\
\mathrm{N} \\
\mathrm{H} \\
\mathrm{H} \\
\mathrm{O} \\
\mathrm{H} \\
\mathrm{O} \\
\mathrm{H}\end{array}$ & $\begin{array}{r}-0.77747200 \\
-0.26571200 \\
-1.80044000 \\
-0.78405400 \\
0.39385600 \\
0.35793900 \\
1.73427900 \\
1.84155700 \\
2.85880500 \\
2.76290800 \\
-0.26368300 \\
-2.15025200 \\
4.16699600 \\
4.94381600 \\
4.36968200 \\
-2.33025300 \\
-1.55369000 \\
-3.32011800 \\
-4.15960500\end{array}$ & $\begin{array}{l} \\
26.493684 \\
1.91197100 \\
2.31645700 \\
2.29699100 \\
0.38671200 \\
-0.32185800 \\
-1.41781700 \\
0.23267500 \\
1.31665200 \\
-0.54965900 \\
-1.63498500 \\
2.31658500 \\
-0.33925600 \\
-0.09446900 \\
-0.73685200 \\
0.89522600 \\
-1.72856800 \\
-2.31277600 \\
0.40930100 \\
-0.08683400\end{array}$ & $\begin{array}{r}0.00004100 \\
-0.88655300 \\
0.00111500 \\
0.00008800 \\
0.00007100 \\
0.00009000 \\
-0.00000300 \\
-0.00012200 \\
0.00001700 \\
0.00007900 \\
0.88538200 \\
0.00000400 \\
-0.00005200 \\
-0.00011000 \\
-0.00008100 \\
-0.00001500 \\
0.00002800 \\
-0.00006500 \\
-0.00013500\end{array}$ \\
\hline$\underbrace{B r}_{H}$ & $\begin{array}{l}\mathrm{C} \\
\mathrm{H} \\
\mathrm{H} \\
\mathrm{C} \\
\mathrm{C} \\
\mathrm{H} \\
\mathrm{C}\end{array}$ & $\begin{array}{l}y:-245.845804 \\
\text { 25.796870 } \\
\text { ted Energy: }-2 \\
y:-245.917762 \\
-0.03935700 \\
-0.62452000 \\
1.03711000 \\
-0.64299400 \\
-2.12431900 \\
-2.66472000 \\
-2.85448100\end{array}$ & $\begin{array}{l} \\
45.807688 \\
2.32614300 \\
3.11907900 \\
2.37225700 \\
1.32688300 \\
1.34411500 \\
1.80404900 \\
0.90587900 \\
\end{array}$ & $\begin{array}{r}-1.39404700 \\
-1.86015200 \\
-1.53817700 \\
-0.68074300 \\
-0.53243700 \\
-1.36186200 \\
0.52299100 \\
\end{array}$ \\
\hline
\end{tabular}




\begin{tabular}{|c|c|c|c|c|}
\hline & $\begin{array}{l}\mathrm{H} \\
\mathrm{C} \\
\mathrm{H} \\
\mathrm{H} \\
\mathrm{B} \\
\mathrm{H} \\
\mathrm{Br} \\
\mathrm{Br}\end{array}$ & $\begin{array}{c}-3.93833800 \\
-2.35684800 \\
-1.27683100 \\
-2.58300500 \\
0.23871100 \\
-2.85831200 \\
2.08149100 \\
-0.37226100\end{array}$ & $\begin{array}{r}1.01518800 \\
0.31257100 \\
0.43531500 \\
-0.76194400 \\
0.13293100 \\
0.79444700 \\
0.39973800 \\
-1.73506900\end{array}$ & $\begin{array}{r}0.45146800 \\
1.81952000 \\
1.95771100 \\
1.87530300 \\
-0.22953500 \\
2.67003200 \\
0.45386900 \\
-0.43839400\end{array}$ \\
\hline $\mathrm{Cl}_{\mathrm{B}}$ & $\begin{array}{l}\text { Fr } \\
\text { En } \\
\text { ZP } \\
\text { To } \\
\\
\text { C } \\
\mathrm{H} \\
\mathrm{H} \\
\mathrm{C} \\
\mathrm{C} \\
\mathrm{H} \\
\mathrm{C} \\
\mathrm{H} \\
\mathrm{C} \\
\mathrm{H} \\
\mathrm{H} \\
\mathrm{B} \\
\mathrm{H} \\
\mathrm{Cl} \\
\mathrm{Cl}\end{array}$ & $\begin{array}{l}y:-249.420473 \\
\text { 249.374048 } \\
\text { ted Energy: }-2 \\
y:-249.495650 \\
0.31330500 \\
-0.29805400 \\
1.37872500 \\
-0.24218900 \\
-1.71068000 \\
-2.15283500 \\
-2.54542800 \\
-3.60018400 \\
-2.21063800 \\
-1.20990500 \\
-2.26836600 \\
0.71985800 \\
-2.93354800 \\
2.31595000 \\
0.38161800\end{array}$ & $\begin{array}{l} \\
249.384432 \\
05 \\
2.38086900 \\
3.25095800 \\
2.55765200 \\
1.13713800 \\
0.97939900 \\
1.65515700 \\
0.13597000 \\
0.17070100 \\
-0.83251200 \\
-0.67414100 \\
-1.87203300 \\
-0.06376300 \\
-0.72725100 \\
0.09620800 \\
-1.67546700\end{array}$ & $\begin{array}{r}-0.42580300 \\
-0.66562100 \\
-0.30189600 \\
-0.29982900 \\
-0.49477500 \\
-1.22897100 \\
0.16095900 \\
-0.11882200 \\
1.27038800 \\
1.68674700 \\
0.91765500 \\
-0.14007700 \\
2.09092500 \\
0.71839500 \\
-0.89164600\end{array}$ \\
\hline${ }^{N C}{ }^{-C N}$ & $\begin{array}{l}\text { Fre } \\
\text { En } \\
\text { ZP } \\
\text { To } \\
\text { C } \\
\mathrm{H} \\
\mathrm{H} \\
\mathrm{C} \\
\mathrm{C} \\
\mathrm{H} \\
\mathrm{C} \\
\mathrm{H} \\
\mathrm{C} \\
\mathrm{H} \\
\mathrm{H} \\
\mathrm{B} \\
\mathrm{H} \\
\mathrm{C} \\
\mathrm{C} \\
\mathrm{N} \\
\mathrm{N}\end{array}$ & $\begin{array}{r}-0.74033600 \\
-0.26736200 \\
-1.81849300 \\
-0.00995600 \\
1.46113100 \\
1.80373300 \\
2.40923100 \\
3.44500800 \\
2.23195000 \\
1.22703400 \\
2.42729200 \\
-0.81475700 \\
2.94942100 \\
-2.26329600 \\
-0.26790000 \\
-3.37360200 \\
0.14248900 \\
\end{array}$ & $\begin{array}{l} \\
05.064278 \\
73 \\
-2.27419300 \\
-3.22850300 \\
-2.29428500 \\
-1.11391200 \\
-1.16537600 \\
-1.93178200 \\
-0.40827600 \\
-0.59988800 \\
0.64264200 \\
0.64569800 \\
1.64809700 \\
0.19938400 \\
0.47077900 \\
0.20346400 \\
1.55655800 \\
0.20493700 \\
2.60185000 \\
\end{array}$ & $\begin{array}{r}-0.36144700 \\
-0.59579600 \\
-0.21904600 \\
-0.27055100 \\
-0.47691300 \\
-1.17474000 \\
0.13054000 \\
-0.15599400 \\
1.20003300 \\
1.63704400 \\
0.80167600 \\
-0.14767100 \\
2.01377800 \\
0.37917200 \\
-0.62180600 \\
0.78984000 \\
-0.99594400 \\
\end{array}$ \\
\hline
\end{tabular}




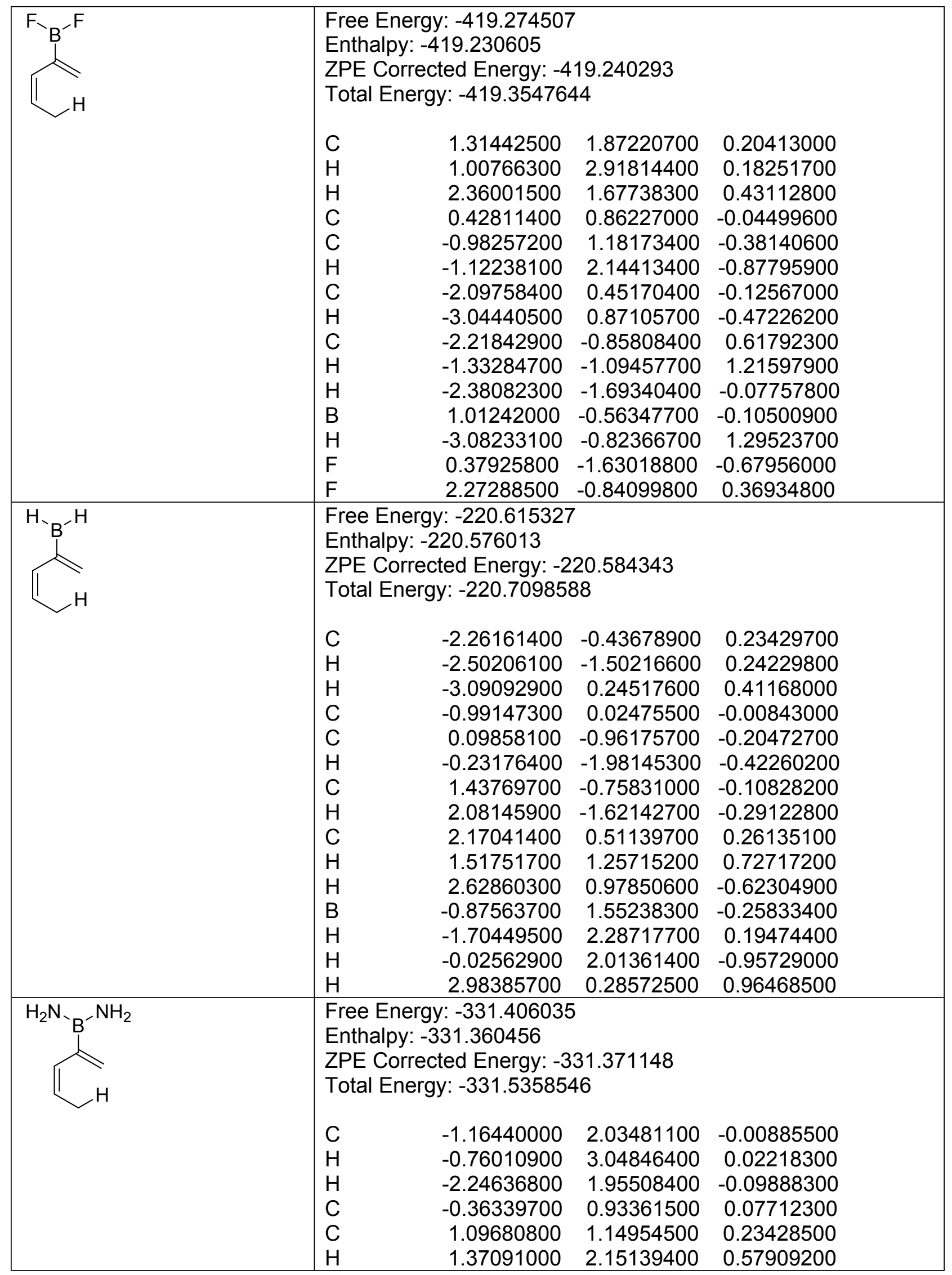




\begin{tabular}{|c|c|c|c|c|}
\hline & $\begin{array}{l}\mathrm{C} \\
\mathrm{H} \\
\mathrm{C} \\
\mathrm{H} \\
\mathrm{H} \\
\mathrm{B} \\
\mathrm{H} \\
\mathrm{N} \\
\mathrm{H} \\
\mathrm{H} \\
\mathrm{N} \\
\mathrm{H} \\
\mathrm{H}\end{array}$ & $\begin{array}{r}2.12800100 \\
3.13386100 \\
2.08774200 \\
1.08322700 \\
2.46627100 \\
-1.05276500 \\
2.74154200 \\
-0.77396600 \\
-0.12711000 \\
-1.19483800 \\
-1.97696900 \\
-2.51549000 \\
-2.14006100\end{array}$ & $\begin{array}{r}0.29983700 \\
0.68242800 \\
-1.09917600 \\
-1.40786800 \\
-1.83492900 \\
-0.49607300 \\
-1.15788300 \\
-1.36958400 \\
-1.09968500 \\
-2.28177900 \\
-0.85082100 \\
-1.70814100 \\
-0.23567800\end{array}$ & $\begin{array}{r}-0.02135100 \\
0.16958400 \\
-0.59327600 \\
-0.88912500 \\
0.13255000 \\
0.12192900 \\
-1.47622500 \\
1.21490700 \\
1.94412100 \\
1.33823400 \\
-0.90709600 \\
-0.92030400 \\
-1.69311100\end{array}$ \\
\hline $\mathrm{HO}_{\mathrm{B}^{-}}-\mathrm{OH}$ & $\begin{array}{l}\mathrm{C} \\
\mathrm{H} \\
\mathrm{H} \\
\mathrm{C} \\
\mathrm{C} \\
\mathrm{H} \\
\mathrm{C} \\
\mathrm{H} \\
\mathrm{C} \\
\mathrm{H} \\
\mathrm{H} \\
\mathrm{B} \\
\mathrm{H} \\
\mathrm{O} \\
\mathrm{H} \\
\mathrm{O} \\
\mathrm{H}\end{array}$ & $\begin{array}{r}1.28427000 \\
0.95572000 \\
2.36112600 \\
0.39748400 \\
-1.04285600 \\
-1.25741900 \\
-2.11247200 \\
-3.09764400 \\
-2.13263000 \\
-1.21254100 \\
-2.26315500 \\
0.99739100 \\
-2.97282500 \\
2.02761900 \\
2.31608900 \\
0.57299500 \\
1.01599700\end{array}$ & $\begin{array}{l}71.145339 \\
08 \\
1.94550100 \\
2.98401200 \\
1.79552100 \\
0.90884300 \\
1.21438900 \\
2.23442200 \\
0.41215400 \\
0.84330100 \\
-1.00770400 \\
-1.28372100 \\
-1.72124100 \\
-0.54064700 \\
-1.14518300 \\
-0.94020500 \\
-0.32824600 \\
-1.45820100 \\
-2.32747700\end{array}$ & $\begin{array}{r}-0.00892700 \\
-0.05781800 \\
0.06155300 \\
-0.07529400 \\
-0.22396100 \\
-0.55349000 \\
0.01577400 \\
-0.17464400 \\
0.53327700 \\
1.05775100 \\
-0.29267800 \\
-0.11449700 \\
1.22724500 \\
0.73713500 \\
1.43693200 \\
-1.05086800 \\
-1.06771400\end{array}$ \\
\hline - & $\begin{array}{l}\mathrm{C} \\
\mathrm{H} \\
\mathrm{H} \\
\mathrm{C} \\
\mathrm{C} \\
\mathrm{H} \\
\mathrm{C} \\
\mathrm{H} \\
\mathrm{C} \\
\mathrm{H}\end{array}$ & $\begin{array}{l}\text { y: }-245.800955 \\
\text { 245.754964 } \\
\text { ted Energy: }-2 \\
\text { y: }-245.871297 \\
-1401.3331 \\
\\
1.82515600 \\
2.44051000 \\
1.24222000 \\
1.19921800 \\
2.04026700 \\
1.57969400 \\
3.36334600 \\
3.77734400 \\
4.06972200 \\
4.36108500\end{array}$ & $\begin{array}{l}\text { l } \\
45.764495 \\
71 \\
-1.35812000 \\
-1.37723800 \\
-2.27191500 \\
-0.10359900 \\
1.00371100 \\
1.96351000 \\
0.79369000 \\
1.51333000 \\
-0.44251300 \\
-0.72218700\end{array}$ & $\begin{array}{r}0.68342500 \\
1.58519200 \\
0.56111500 \\
0.33190300 \\
-0.00614800 \\
-0.22523600 \\
-0.41726900 \\
-1.12384800 \\
-0.20600100 \\
0.80752200\end{array}$ \\
\hline
\end{tabular}




\begin{tabular}{|c|c|c|c|c|}
\hline & $\begin{array}{l}\mathrm{H} \\
\mathrm{B} \\
\mathrm{H} \\
\mathrm{Br} \\
\mathrm{Br}\end{array}$ & $\begin{array}{r}2.97159000 \\
-0.31164800 \\
4.84874600 \\
-1.26117500 \\
-1.44308800\end{array}$ & $\begin{array}{r}-1.30541800 \\
-0.01458300 \\
-0.68696500 \\
1.73052400 \\
-1.62764500\end{array}$ & $\begin{array}{r}-0.12183400 \\
0.09353100 \\
-0.93331200 \\
0.06141800 \\
-0.15663900\end{array}$ \\
\hline ; & $\begin{array}{l}\mathrm{C} \\
\mathrm{H} \\
\mathrm{H} \\
\mathrm{C} \\
\mathrm{C} \\
\mathrm{H} \\
\mathrm{C} \\
\mathrm{H} \\
\mathrm{C} \\
\mathrm{H} \\
\mathrm{H} \\
\mathrm{B} \\
\mathrm{H} \\
\mathrm{Cl} \\
\mathrm{Cl}\end{array}$ & $\begin{array}{r}1.13672000 \\
1.74181400 \\
0.55189200 \\
0.51557300 \\
1.35264900 \\
0.88752500 \\
2.68358600 \\
3.10474700 \\
3.39084200 \\
3.66452200 \\
2.29548600 \\
-0.98770700 \\
4.18393300 \\
-1.85032300 \\
-2.03012500 \\
\end{array}$ & $\begin{array}{l} \\
49.341083 \\
09 \\
-1.39942900 \\
-1.53429100 \\
-2.28824500 \\
-0.11172500 \\
1.03211800 \\
2.00835600 \\
0.87826600 \\
1.67880800 \\
-0.37291600 \\
-0.77452300 \\
-1.24414700 \\
0.00151600 \\
-0.52514000 \\
1.60364100 \\
-1.45577500 \\
\end{array}$ & $\begin{array}{r}0.50848600 \\
1.40719300 \\
0.26762300 \\
0.30419800 \\
0.11524800 \\
0.00421900 \\
-0.29620700 \\
-0.90451800 \\
-0.22498500 \\
0.75180900 \\
-0.26769100 \\
0.05252900 \\
-0.96214800 \\
0.08605300 \\
-0.26249800 \\
\end{array}$ \\
\hline NC: & $\begin{array}{l}\text { Fre } \\
\text { En } \\
\text { ZP } \\
\text { To } \\
\text { Fre } \\
\text { C } \\
\mathrm{H} \\
\mathrm{H} \\
\mathrm{C} \\
\mathrm{C} \\
\mathrm{H} \\
\mathrm{C} \\
\mathrm{H} \\
\mathrm{C} \\
\mathrm{H} \\
\mathrm{H} \\
\mathrm{B} \\
\mathrm{H} \\
\mathrm{C} \\
\mathrm{C} \\
\mathrm{N} \\
\mathrm{N}\end{array}$ & $\begin{array}{l}\text { y: }-405.058926 \\
\text { 05.013032 } \\
\text { ted Energy: }-40 \\
y:-405.145987 \\
-1351.0630 \\
0.96085700 \\
1.53867900 \\
0.38153400 \\
0.35070800 \\
1.19831800 \\
0.73966000 \\
2.52511000 \\
2.96671700 \\
3.21482300 \\
3.49421500 \\
2.12125300 \\
-1.14848100 \\
3.99188800 \\
-2.03470300 \\
-1.85540100 \\
-2.70698900 \\
-2.38584200 \\
\end{array}$ & $\begin{array}{l} \\
05.023559 \\
76 \\
-1.40262400 \\
-1.54963300 \\
-2.28843300 \\
-0.10700000 \\
1.03460500 \\
2.01475200 \\
0.86562300 \\
1.65826700 \\
-0.39848700 \\
-0.78517300 \\
-1.26155200 \\
0.01359300 \\
-0.58168600 \\
-1.22582000 \\
1.38402500 \\
-2.18188000 \\
2.44238800 \\
\end{array}$ & $\begin{array}{r}0.49975700 \\
1.41476900 \\
0.23150600 \\
0.28584900 \\
0.10127800 \\
-0.00765900 \\
-0.30377500 \\
-0.90734400 \\
-0.22051300 \\
0.76073900 \\
-0.25831100 \\
0.05431300 \\
-0.96748100 \\
-0.17844400 \\
0.06832100 \\
-0.36737400 \\
0.07414200 \\
\end{array}$ \\
\hline
\end{tabular}




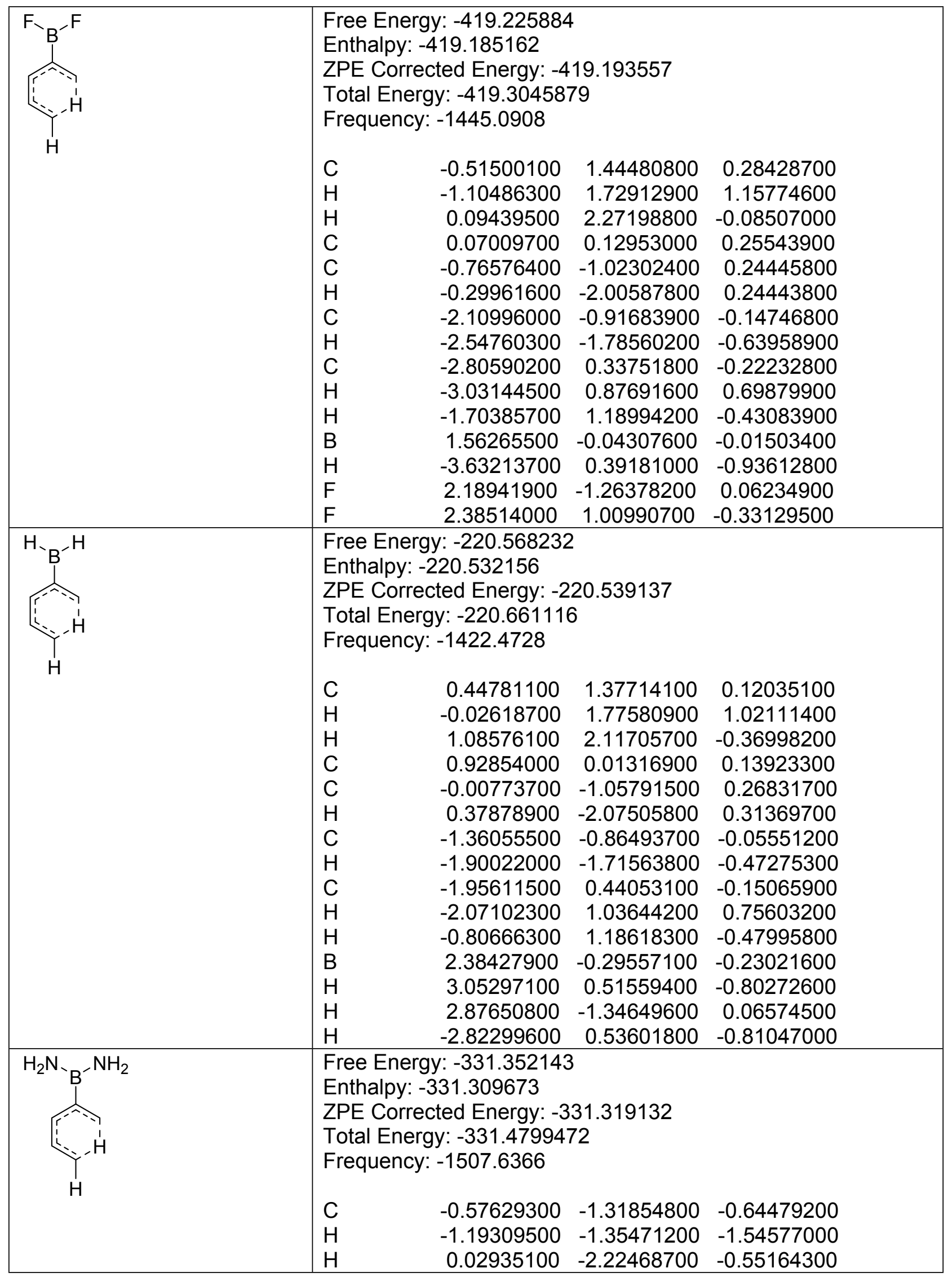




\begin{tabular}{|c|c|c|c|c|}
\hline & $\begin{array}{l}\text { C } \\
\mathrm{C} \\
\mathrm{H} \\
\mathrm{C} \\
\mathrm{H} \\
\mathrm{C} \\
\mathrm{H} \\
\mathrm{H} \\
\mathrm{B} \\
\mathrm{H} \\
\mathrm{N} \\
\mathrm{H} \\
\mathrm{H} \\
\mathrm{N} \\
\mathrm{H} \\
\mathrm{H}\end{array}$ & $\begin{array}{r}0.04865900 \\
-0.76698900 \\
-0.28108500 \\
-2.11781000 \\
-2.51712500 \\
-2.86069500 \\
-3.08603400 \\
-1.76893100 \\
1.59766900 \\
-3.71073300 \\
2.25849700 \\
3.25293400 \\
1.75336000 \\
2.31332900 \\
1.87077300 \\
3.29823600\end{array}$ & $\begin{array}{r}-0.07193700 \\
1.04762100 \\
1.98143800 \\
0.88394800 \\
1.60584400 \\
-0.31916100 \\
-0.60558500 \\
-1.22804900 \\
0.00739300 \\
-0.50346100 \\
-1.08436100 \\
-1.10639000 \\
-1.89275100 \\
1.18481900 \\
1.92644300 \\
1.33021000\end{array}$ & $\begin{array}{r}-0.29345800 \\
0.02085900 \\
0.30178300 \\
0.38910500 \\
1.10359500 \\
0.14563000 \\
-0.88318900 \\
0.12588300 \\
0.01784300 \\
0.80877700 \\
0.66020900 \\
0.84713900 \\
0.99698300 \\
-0.36391200 \\
-0.88984100 \\
-0.18107700\end{array}$ \\
\hline HO & $\begin{array}{l}\mathrm{C} \\
\mathrm{H} \\
\mathrm{H} \\
\mathrm{C} \\
\mathrm{C} \\
\mathrm{H} \\
\mathrm{C} \\
\mathrm{H} \\
\mathrm{C} \\
\mathrm{H} \\
\mathrm{H} \\
\mathrm{B} \\
\mathrm{H} \\
\mathrm{O} \\
\mathrm{H} \\
\mathrm{O} \\
\mathrm{H}\end{array}$ & $\begin{array}{l}y:-371.130449 \\
371.089018 \\
\text { ted Energy: }-3 \\
y:-371.23313 \\
-1482.5076 \\
-0.54502000 \\
-1.14200500 \\
0.04851400 \\
0.05659600 \\
-0.77369300 \\
-0.28940300 \\
-2.12189200 \\
-2.54213900 \\
-2.84035100 \\
-3.05875600 \\
-1.74300600 \\
1.57984600 \\
-3.68410700 \\
2.46850200 \\
2.14868600 \\
2.11108600 \\
3.07244900\end{array}$ & $\begin{array}{l} \\
71.097873 \\
77 \\
1.45593100 \\
1.76853800 \\
2.29310500 \\
0.14805200 \\
-1.00379900 \\
-1.97686900 \\
-0.92542900 \\
-1.81288000 \\
0.31257700 \\
0.87657100 \\
1.16785600 \\
-0.05456100 \\
0.32653700 \\
0.98478800 \\
1.90317000 \\
-1.33131400 \\
-1.42500800\end{array}$ & $\begin{array}{r}0.23737900 \\
1.09669700 \\
-0.14837100 \\
0.25205800 \\
0.27001500 \\
0.28837000 \\
-0.12479600 \\
-0.59993200 \\
-0.22812100 \\
0.68060700 \\
-0.46732800 \\
-0.01096800 \\
-0.92341600 \\
-0.27100800 \\
-0.26909000 \\
0.02861200 \\
-0.10272700\end{array}$ \\
\hline (N-B & $\begin{array}{l}\mathrm{C} \\
\mathrm{H} \\
\mathrm{H} \\
\mathrm{C} \\
\mathrm{C} \\
\mathrm{H}\end{array}$ & $\begin{array}{l}y:-295.814132 \\
295.771819 \\
\text { ted Energy: }-2 \\
\text { y: }-295.91108 \\
\\
2.82130200 \\
3.10143800 \\
3.61288000 \\
1.54748700 \\
0.51256400 \\
0.89300100\end{array}$ & $\begin{array}{l} \\
95.781167 \\
52 \\
-0.36395300 \\
-1.41929500 \\
0.35006300 \\
0.04777700 \\
-0.98708400 \\
-2.00558400\end{array}$ & $\begin{array}{r}-0.32781900 \\
-0.35248000 \\
-0.54593500 \\
-0.02000700 \\
0.21848000 \\
0.33189700\end{array}$ \\
\hline
\end{tabular}




\begin{tabular}{|c|c|c|c|c|}
\hline & $\begin{array}{l}\mathrm{C} \\
\mathrm{H} \\
\mathrm{C} \\
\mathrm{H} \\
\mathrm{H} \\
\mathrm{O} \\
\mathrm{H} \\
\mathrm{B} \\
\mathrm{H} \\
\mathrm{H}\end{array}$ & $\begin{array}{c}-0.83309000 \\
-1.45428700 \\
-1.64330800 \\
-1.16670900 \\
-1.74851100 \\
-2.95863600 \\
-3.56220900 \\
1.37572200 \\
2.18625800 \\
0.49888100 \\
\end{array}$ & $\begin{array}{r}-0.83396900 \\
-1.71153300 \\
0.42609700 \\
1.11947100 \\
0.94950900 \\
-0.00020500 \\
0.76731100 \\
1.57329600 \\
2.32682500 \\
2.02518100 \\
\end{array}$ & $\begin{array}{r}0.27562700 \\
0.45331600 \\
0.10649200 \\
-0.60374800 \\
1.07193500 \\
-0.38423700 \\
-0.46799200 \\
0.20744900 \\
-0.24606000 \\
0.87907400 \\
\end{array}$ \\
\hline = & $\begin{array}{l}\mathrm{C} \\
\mathrm{H} \\
\mathrm{H} \\
\mathrm{C} \\
\mathrm{C} \\
\mathrm{H} \\
\mathrm{C} \\
\mathrm{H} \\
\mathrm{C} \\
\mathrm{H} \\
\mathrm{H} \\
\mathrm{O} \\
\mathrm{H} \\
\mathrm{B} \\
\mathrm{H} \\
\mathrm{H}\end{array}$ & $\begin{array}{l}y:-295.777372 \\
295.738389 \\
\text { ted Energy: }-2 \\
y:-295.872613 \\
-1343.9905 \\
0.83630200 \\
0.43520300 \\
1.30341200 \\
1.45533000 \\
0.63826500 \\
1.10609000 \\
-0.75360000 \\
-1.32317100 \\
-1.48163000 \\
-1.61015200 \\
-0.46854900 \\
-2.67913100 \\
-3.28021200 \\
2.85416600 \\
3.36562000 \\
3.46598100\end{array}$ & $\begin{array}{l} \\
95.746557 \\
32 \\
1.34259100 \\
1.58373100 \\
2.20509400 \\
0.05656400 \\
-1.10473900 \\
-2.08598900 \\
-1.03287600 \\
-1.91934000 \\
0.21873100 \\
0.76127700 \\
1.06442100 \\
0.14417600 \\
0.89663500 \\
-0.06413200 \\
0.87027400 \\
-1.09047600\end{array}$ & $\begin{array}{r}0.28760900 \\
1.27768200 \\
-0.19403800 \\
0.09289500 \\
0.28245700 \\
0.22707200 \\
0.22352700 \\
-0.05006000 \\
0.21380700 \\
1.15843100 \\
-0.19462200 \\
-0.55174200 \\
-0.37469200 \\
-0.51075300 \\
-1.05764100 \\
-0.42620100\end{array}$ \\
\hline $\mathrm{NH}_{2}$ & $\begin{array}{l}\mathrm{C} \\
\mathrm{H} \\
\mathrm{H} \\
\mathrm{C} \\
\mathrm{H} \\
\mathrm{C} \\
\mathrm{H} \\
\mathrm{C} \\
\mathrm{H} \\
\mathrm{C} \\
\mathrm{H} \\
\mathrm{H} \\
\mathrm{N}\end{array}$ & $\begin{array}{r}2.96686400 \\
3.49251500 \\
3.57544800 \\
1.61992000 \\
1.14334200 \\
0.75787800 \\
1.27269900 \\
-0.58890200 \\
-1.10412800 \\
-1.51512100 \\
-1.86028200 \\
-0.97490700 \\
-2.71026400 \\
\end{array}$ & $\begin{array}{r}-0.35901000 \\
0.58196900 \\
-1.25939000 \\
-0.40199600 \\
-1.36903000 \\
0.78531300 \\
1.72910100 \\
0.82323100 \\
1.78219300 \\
-0.33969100 \\
-0.26073800 \\
-1.29919800 \\
-0.25887600 \\
\end{array}$ & $\begin{array}{r}0.13733800 \\
0.29402800 \\
0.10949300 \\
-0.01834400 \\
-0.17939400 \\
0.01783700 \\
0.20936800 \\
-0.16361700 \\
-0.10122000 \\
-0.45156500 \\
-1.49409800 \\
-0.37055200 \\
0.41016500 \\
\end{array}$ \\
\hline
\end{tabular}




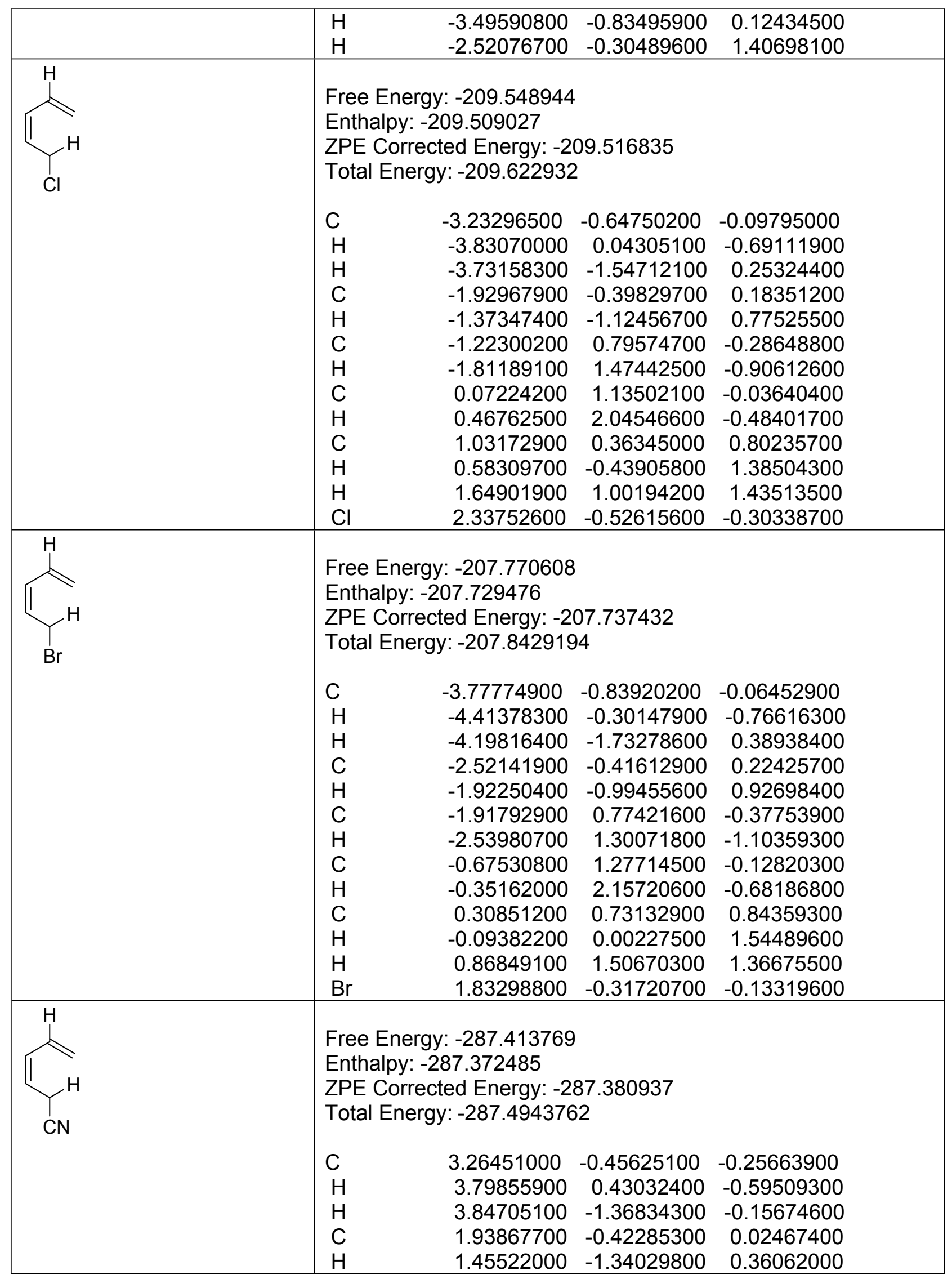




\begin{tabular}{|c|c|c|c|c|}
\hline & $\begin{array}{l}\mathrm{C} \\
\mathrm{H} \\
\mathrm{C} \\
\mathrm{H} \\
\mathrm{C} \\
\mathrm{H} \\
\mathrm{H} \\
\mathrm{C} \\
\mathrm{N}\end{array}$ & $\begin{array}{r}1.11732100 \\
1.64300100 \\
-0.20490400 \\
-0.68236100 \\
-1.11970100 \\
-0.63150200 \\
-1.37847500 \\
-2.38186700 \\
-3.39081600 \\
\end{array}$ & $\begin{array}{r}0.78602000 \\
1.67286600 \\
0.91475700 \\
1.88239100 \\
-0.17874400 \\
-1.15885900 \\
0.03060600 \\
-0.27233300 \\
-0.33889500 \\
\end{array}$ & $\begin{array}{r}-0.10293400 \\
-0.46083300 \\
0.17398900 \\
0.02684700 \\
0.71039100 \\
0.69485300 \\
1.75978500 \\
-0.05106000 \\
-0.65999300 \\
\end{array}$ \\
\hline$Y_{\mathrm{Cl}}$ & $\begin{array}{l}\text { Fre } \\
\text { En } \\
\text { ZP } \\
\text { Tot } \\
\text { Fre } \\
\text { C } \\
\mathrm{H} \\
\mathrm{H} \\
\mathrm{C} \\
\mathrm{H} \\
\mathrm{C} \\
\mathrm{H} \\
\mathrm{C} \\
\mathrm{H} \\
\mathrm{C} \\
\mathrm{H} \\
\mathrm{H} \\
\mathrm{Cl}\end{array}$ & $\begin{array}{l}\text { : }-209.491173 \\
09.454506 \\
\text { ted Energy: }-2 \\
y:-209.562766 \\
-1475.7873 \\
-1.73886100 \\
-1.50160800 \\
-2.17441900 \\
-2.29137900 \\
-3.19336900 \\
-1.54999000 \\
-1.99857700 \\
-0.15331200 \\
0.42766900 \\
0.53784500 \\
0.51840200 \\
-0.39374200 \\
2.32293100\end{array}$ & $\begin{array}{l} \\
09.461214 \\
4 \\
-1.33429700 \\
-1.61037200 \\
-2.16960300 \\
-0.03527500 \\
0.06595600 \\
1.13251100 \\
2.10459100 \\
1.07045800 \\
1.96197200 \\
-0.16915100 \\
-0.73017700 \\
-1.05209500 \\
-0.15033800\end{array}$ & $\begin{array}{r}0.01058700 \\
1.04170700 \\
-0.54176800 \\
-0.22781700 \\
-0.83064200 \\
0.03368700 \\
-0.15854900 \\
0.21385500 \\
-0.01362200 \\
0.38092100 \\
1.31295000 \\
-0.22617700 \\
-0.17948800\end{array}$ \\
\hline $\mathrm{Br}_{\mathrm{Br}}$ & $\begin{array}{l}\text { Fre } \\
\text { En } \\
\text { ZP } \\
\text { Tot } \\
\text { Fre } \\
\text { C } \\
\mathrm{H} \\
\mathrm{H} \\
\mathrm{C} \\
\mathrm{H} \\
\mathrm{C} \\
\mathrm{H} \\
\mathrm{C} \\
\mathrm{H} \\
\mathrm{C} \\
\mathrm{H} \\
\mathrm{H} \\
\mathrm{Br}\end{array}$ & $\begin{array}{r}-2.34689000 \\
-2.14226200 \\
-2.73270800 \\
-2.93284400 \\
-3.81503500 \\
-2.24154900 \\
-2.71595100 \\
-0.84970600 \\
-0.29797000 \\
-0.12415800 \\
-0.16496900 \\
-1.00465400 \\
1.82412700\end{array}$ & $\begin{array}{l} \\
07.680754 \\
\\
-1.35943500 \\
-1.61846400 \\
-2.21367600 \\
-0.08192300 \\
-0.01825800 \\
1.11362800 \\
2.06738300 \\
1.10264600 \\
2.01384200 \\
-0.11238000 \\
-0.65701700 \\
-1.03414100 \\
-0.07185400\end{array}$ & $\begin{array}{r}0.01128100 \\
1.05364500 \\
-0.54920200 \\
-0.26272000 \\
-0.89921300 \\
0.00991100 \\
-0.21052200 \\
0.23755000 \\
0.01439600 \\
0.44654100 \\
1.38787500 \\
-0.17592200 \\
-0.09361200\end{array}$ \\
\hline
\end{tabular}




\begin{tabular}{|c|c|c|c|c|}
\hline \multirow[t]{15}{*}{ 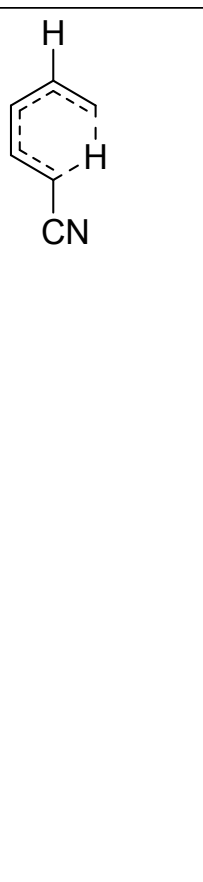 } & \multicolumn{4}{|c|}{$\begin{array}{l}\text { Free Energy: -287.361694 } \\
\text { Enthalpy: -287.324163 } \\
\text { ZPE Corrected Energy: -287.331384 } \\
\text { Total Energy: -287.4410583 } \\
\text { Frequency: -1524.8355 }\end{array}$} \\
\hline & C & -1.59958100 & -1.36609500 & -0.00072900 \\
\hline & $\mathrm{H}$ & -1.34044500 & -1.64417800 & 1.02256300 \\
\hline & $\mathrm{H}$ & -2.03240800 & -2.20119500 & -0.55414500 \\
\hline & C & -2.14800000 & -0.07451300 & -0.24932500 \\
\hline & $\mathrm{H}$ & -3.02891100 & 0.01253500 & -0.88607500 \\
\hline & C & -1.44137300 & 1.12383700 & 0.01657100 \\
\hline & $\mathrm{H}$ & -1.92140300 & 2.07471600 & -0.20299400 \\
\hline & C & -0.05076400 & 1.10323400 & 0.18203400 \\
\hline & $\mathrm{H}$ & 0.50604900 & 2.00089800 & -0.08139000 \\
\hline & C & 0.68348300 & -0.12497900 & 0.40572300 \\
\hline & $\mathrm{H}$ & 0.53441900 & -0.63869000 & 1.35881700 \\
\hline & $\mathrm{H}$ & -0.22029400 & -1.03507300 & -0.20084500 \\
\hline & C & 2.07502100 & -0.15747600 & -0.00199000 \\
\hline & $\mathrm{N}$ & 3.19861100 & -0.22758100 & -0.36709100 \\
\hline \multirow[t]{16}{*}{$\mathrm{NH}_{2}$} & \multicolumn{4}{|c|}{$\begin{array}{l}\text { Free Energy: }-250.468481 \\
\text { Enthalpy: }-250.432134 \\
\text { ZPE Corrected Energy: }-250.439213 \\
\text { Total Energy: }-250.5670117 \\
\text { Frequency: }-1456.9076\end{array}$} \\
\hline & C & -1.34960400 & -1.29903200 & 0.01464000 \\
\hline & $\mathrm{H}$ & -1.03127900 & -1.59157600 & 1.01846500 \\
\hline & $\mathrm{H}$ & -1.84528500 & -2.11861100 & -0.51232400 \\
\hline & C & -1.85824800 & 0.01754600 & -0.18623800 \\
\hline & $\mathrm{H}$ & -2.77182000 & 0.16651200 & -0.76138900 \\
\hline & C & -1.04396200 & 1.14549400 & 0.06611400 \\
\hline & $\mathrm{H}$ & -1.46158200 & 2.14092400 & -0.07917600 \\
\hline & C & 0.34609100 & 1.02839400 & 0.21160300 \\
\hline & $\mathrm{H}$ & 0.95396300 & 1.91689700 & 0.03557100 \\
\hline & C & 1.05209300 & -0.24201200 & 0.26854400 \\
\hline & $\mathrm{H}$ & 0.98981300 & -0.81390700 & 1.19847400 \\
\hline & $\mathrm{H}$ & 0.05316300 & -1.04250500 & -0.29415200 \\
\hline & $\mathrm{N}$ & 2.38100800 & -0.25261700 & -0.24659900 \\
\hline & $\mathrm{H}$ & 3.00270500 & -1.01120900 & -0.00596400 \\
\hline & $\mathrm{H}$ & 2.56505100 & 0.21944700 & -1.12129100 \\
\hline \multirow[t]{5}{*}{$\mathrm{H}_{3} \mathrm{C} \backslash \mathrm{CH}_{2}$} & \multicolumn{4}{|c|}{$\begin{array}{l}\text { Free Energy: }-117.836172 \\
\text { Enthalpy: -117.806070 } \\
\text { ZPE Corrected Energy: }-117.811106 \\
\text { Total Energy: }-117.8913038\end{array}$} \\
\hline & C & 1.24596700 & -0.16548300 & 0.00000100 \\
\hline & $\mathrm{H}$ & 1.18930500 & -1.26032500 & 0.00024900 \\
\hline & $\mathrm{H}$ & 1.82033900 & 0.14935300 & 0.88339500 \\
\hline & C & -0.13130000 & 0.45928500 & 0.00005400 \\
\hline
\end{tabular}




\begin{tabular}{|c|c|c|c|c|}
\hline & $\begin{array}{l}\mathrm{H} \\
\mathrm{C} \\
\mathrm{H} \\
\mathrm{H} \\
\mathrm{H}\end{array}$ & $\begin{array}{r}-0.16118700 \\
-1.29627400 \\
-2.25619700 \\
-1.32254600 \\
1.81992700\end{array}$ & $\begin{array}{r}1.55154900 \\
-0.22195900 \\
0.29009700 \\
-1.31060400 \\
0.14887300\end{array}$ & $\begin{array}{r}-0.00012500 \\
0.00003400 \\
-0.00014800 \\
-0.00006800 \\
-0.88383700\end{array}$ \\
\hline $\mathrm{Br} \frown=\mathrm{CH}_{2}$ & $\begin{array}{l}01 \\
\mathrm{C} \\
\mathrm{H} \\
\mathrm{H} \\
\mathrm{C} \\
\mathrm{H} \\
\mathrm{C} \\
\mathrm{H} \\
\mathrm{H} \\
\mathrm{Br}\end{array}$ & $\begin{array}{l}y:-130.409333 \\
130.374157 \\
\text { ted Energy: }-1 \\
\text { y: }-130.451469 \\
\\
0.64591600 \\
0.78187700 \\
0.43108700 \\
1.75222500 \\
1.70328700 \\
2.78310700 \\
3.58251700 \\
2.85485300 \\
-1.15546000\end{array}$ & $\begin{array}{l}30.380060 \\
0.89192800 \\
1.15583400 \\
1.77494000 \\
0.05297500 \\
-0.17631500 \\
-0.40541000 \\
-0.99401000 \\
-0.20587200 \\
-0.13690100\end{array}$ & $\begin{array}{r}-0.06892200 \\
-1.11828300 \\
0.53508600 \\
0.47958700 \\
1.54371900 \\
-0.26359500 \\
0.17958900 \\
-1.33139900 \\
-0.01974700\end{array}$ \\
\hline $\mathrm{Cl} \stackrel{\ominus}{=} \mathrm{CH}_{2}$ & $\begin{array}{l}02 \\
\mathrm{C} \\
\mathrm{H} \\
\mathrm{C} \\
\mathrm{H} \\
\mathrm{C} \\
\mathrm{H} \\
\mathrm{H} \\
\mathrm{Cl}\end{array}$ & $\begin{array}{l}\text { y: }-131.555543 \\
131.521620 \\
\text { ted Energy: }-1 \\
\text { y: }-131.58505 \\
\\
0.03075600 \\
0.09984700 \\
1.10730900 \\
0.88279900 \\
2.44509500 \\
3.24663800 \\
2.72617200 \\
-1.67378900\end{array}$ & $\begin{array}{r}0.51152300 \\
1.59284900 \\
-0.37680800 \\
-1.44289300 \\
0.02646200 \\
-0.70614600 \\
1.07739300 \\
-0.08754500\end{array}$ & $\begin{array}{r}-0.00045800 \\
0.00039500 \\
-0.00022400 \\
-0.00046600 \\
0.00022300 \\
0.00057100 \\
0.00014000 \\
0.00012500\end{array}$ \\
\hline $\mathrm{Cl} \widehat{\sim}=\mathrm{CH}_{2}$ & $\begin{array}{l}01 \\
\mathrm{C} \\
\mathrm{H} \\
\mathrm{H} \\
\mathrm{C} \\
\mathrm{H} \\
\mathrm{C} \\
\mathrm{H} \\
\mathrm{H} \\
\mathrm{Cl}\end{array}$ & $\begin{array}{r}0.00650300 \\
0.13808300 \\
-0.26276500 \\
1.16269000 \\
1.09897700 \\
2.24789700 \\
3.08022900 \\
2.33385000 \\
-1.58181900\end{array}$ & $\begin{array}{l}32.159871 \\
11 \\
0.79964400 \\
1.21737600 \\
1.57600200 \\
-0.03677400 \\
-0.45229900 \\
-0.27661400 \\
-0.87183000 \\
0.11378800 \\
-0.26474000\end{array}$ & $\begin{array}{r}0.03136500 \\
-0.96730600 \\
0.74950300 \\
0.47557100 \\
1.48066100 \\
-0.29063100 \\
0.07652400 \\
-1.30323300 \\
-0.07846900\end{array}$ \\
\hline $\mathrm{NC} \stackrel{\wedge}{=} \mathrm{CH}_{2}$ & \multicolumn{4}{|c|}{ Free Energy: -209.435576 } \\
\hline
\end{tabular}




\begin{tabular}{|c|c|c|c|c|}
\hline & $\begin{array}{l}02 \\
\mathrm{C} \\
\mathrm{H} \\
\mathrm{C} \\
\mathrm{H} \\
\mathrm{C} \\
\mathrm{H} \\
\mathrm{H} \\
\mathrm{C} \\
\mathrm{N}\end{array}$ & $\begin{array}{r}-0.06785000 \\
0.12393700 \\
1.01801000 \\
0.78298500 \\
2.34773700 \\
3.15068800 \\
2.63076900 \\
-1.42056400 \\
-2.56462500\end{array}$ & $\begin{array}{l}09.407123 \\
75 \\
0.51075200 \\
1.58269500 \\
-0.40068600 \\
-1.46439300 \\
-0.00864300 \\
-0.73992000 \\
1.04229900 \\
0.09865100 \\
-0.23158900\end{array}$ & $\begin{array}{r}0.00008000 \\
-0.00029900 \\
-0.00015500 \\
0.00009100 \\
0.00003000 \\
0.00016800 \\
0.00017800 \\
-0.00001900 \\
0.00003500\end{array}$ \\
\hline $\mathrm{NC} \Upsilon=\mathrm{CH}_{2}$ & $\begin{array}{l}01 \\
\mathrm{C} \\
\mathrm{H} \\
\mathrm{H} \\
\mathrm{C} \\
\mathrm{H} \\
\mathrm{C} \\
\mathrm{H} \\
\mathrm{H} \\
\mathrm{C} \\
\mathrm{N}\end{array}$ & $\begin{array}{r}-0.08853400 \\
0.12264000 \\
-0.19555600 \\
1.05407500 \\
0.90440500 \\
2.20014600 \\
2.99659700 \\
2.37617200 \\
-1.38296800 \\
-2.41436800\end{array}$ & $\begin{array}{l}l \\
10.024609 \\
54 \\
0.75063200 \\
1.35267100 \\
1.44812000 \\
-0.22125800 \\
-0.91310200 \\
-0.24555300 \\
-0.94463900 \\
0.42445600 \\
0.06329000 \\
-0.49288100\end{array}$ & $\begin{array}{r}0.13410100 \\
-0.75816600 \\
0.97826200 \\
0.40144300 \\
1.23018900 \\
-0.30456800 \\
-0.06225000 \\
-1.14443300 \\
-0.04906600 \\
-0.19072400\end{array}$ \\
\hline$\stackrel{\mathrm{P}}{\mathrm{Y}}=\mathrm{CH}_{2}$ & $\begin{array}{l}02 \\
\mathrm{C} \\
\mathrm{H} \\
\mathrm{F} \\
\mathrm{C} \\
\mathrm{H} \\
\mathrm{C} \\
\mathrm{H} \\
\mathrm{H}\end{array}$ & $\begin{array}{r}-0.54080300 \\
-0.57354000 \\
-1.81482500 \\
0.58290800 \\
0.40216900 \\
1.89848600 \\
2.73734600 \\
2.12390000\end{array}$ & $\begin{array}{r}0.43048800 \\
1.51536100 \\
-0.15062800 \\
-0.38894900 \\
-1.46355700 \\
0.08489800 \\
-0.60382500 \\
1.14904600\end{array}$ & $\begin{array}{r}-0.00010600 \\
0.00008100 \\
0.00003300 \\
0.00000900 \\
0.00005000 \\
0.00002800 \\
-0.00016600 \\
0.00015200\end{array}$ \\
\hline $\mathrm{F} \widehat{=}=\mathrm{CH}_{2}$ & \multicolumn{4}{|c|}{$\begin{array}{l}\text { Free Energy: }-217.085346 \\
\text { Enthalpy: }-217.052688 \\
\text { ZPE Corrected Energy: }-217.058241 \\
\text { Total Energy: }-217.1314698 \\
\text { Frequency: } 110.7142 \\
01\end{array}$} \\
\hline
\end{tabular}




\begin{tabular}{|c|c|c|c|c|}
\hline & $\begin{array}{l}\mathrm{C} \\
\mathrm{H} \\
\mathrm{H} \\
\mathrm{F} \\
\mathrm{C} \\
\mathrm{H} \\
\mathrm{C} \\
\mathrm{H} \\
\mathrm{H}\end{array}$ & $\begin{array}{r}-0.59268500 \\
-0.47480000 \\
-0.94265100 \\
-1.68247000 \\
0.65503400 \\
0.56884100 \\
1.82757900 \\
2.71657600 \\
1.93470100 \\
\end{array}$ & $\begin{array}{r}0.47157800 \\
1.42275400 \\
0.64548400 \\
-0.25459700 \\
-0.36082200 \\
-1.35986300 \\
0.06935400 \\
-0.55624600 \\
1.05858300 \\
\end{array}$ & $\begin{array}{r}0.31551700 \\
-0.21451000 \\
1.34071200 \\
-0.34492200 \\
0.27636000 \\
0.70328400 \\
-0.23104900 \\
-0.21662700 \\
-0.67352800 \\
\end{array}$ \\
\hline $\mathrm{HO} \stackrel{\bullet}{\wedge}=\mathrm{CH}_{2}$ & $\begin{array}{l}\mathrm{Fr} \\
\mathrm{Er} \\
\mathrm{ZF} \\
\mathrm{Tc} \\
\mathrm{Fr} \\
\\
0 \\
\mathrm{C} \\
\mathrm{H} \\
\mathrm{C} \\
\mathrm{H} \\
\mathrm{C} \\
\mathrm{H} \\
\mathrm{H} \\
\mathrm{O} \\
\mathrm{H}\end{array}$ & $\begin{array}{l}y:-192.414336 \\
192.381090 \\
\text { ted Energy: }-1 \\
214.2452 \\
\\
-0.53622400 \\
-0.50847000 \\
0.61409000 \\
0.45035000 \\
1.92096300 \\
2.76995600 \\
2.13327500 \\
-1.78855800 \\
-2.52962600\end{array}$ & $\begin{array}{l} \\
92.387001 \\
27 \\
\\
0.40669100 \\
1.49617600 \\
-0.38793600 \\
-1.46558300 \\
0.10118100 \\
-0.57539900 \\
1.16853500 \\
-0.22007200 \\
0.41723300\end{array}$ & $\begin{array}{r}0.00049000 \\
0.00095000 \\
0.00036900 \\
0.00099200 \\
-0.00033300 \\
-0.00017000 \\
-0.00103700 \\
-0.00051600 \\
0.00024200\end{array}$ \\
\hline $\mathrm{HO} \widehat{=}=\mathrm{CH}_{2}$ & $\begin{array}{l}0 \\
\mathrm{C} \\
\mathrm{H} \\
\mathrm{H} \\
\mathrm{C} \\
\mathrm{H} \\
\mathrm{C} \\
\mathrm{H} \\
\mathrm{H} \\
\mathrm{O} \\
\mathrm{H}\end{array}$ & $\begin{array}{r}1.93533900 \\
2.21264100 \\
2.75349700 \\
0.65188800 \\
0.40310200 \\
-0.55803500 \\
-0.55344000 \\
-0.55404500 \\
-1.73438300 \\
-2.56184400\end{array}$ & $\begin{array}{r}0.03496500 \\
1.08861000 \\
-0.68077600 \\
-0.37444300 \\
-1.43518400 \\
0.53541600 \\
1.18315300 \\
1.18216300 \\
-0.33749100 \\
0.18633400\end{array}$ & $\begin{array}{r}-0.00035200 \\
-0.00101100 \\
-0.00027200 \\
0.00032500 \\
0.00094800 \\
0.00040600 \\
-0.89157100 \\
0.89311400 \\
-0.00049200 \\
0.00045400\end{array}$ \\
\hline $\mathrm{H}_{2} \mathrm{~N} \stackrel{\ominus}{=} \mathrm{CH}_{2}$ & \multicolumn{4}{|c|}{$\begin{array}{l}\text { Free Energy: }-172.545137 \\
\text { Enthalpy: }-172.511254 \\
\text { ZPE Corrected Energy: }-172.517549 \\
\text { Total Energy: }-172.6015758 \\
\text { Frequency: } 204.1987\end{array}$} \\
\hline
\end{tabular}




\begin{tabular}{|c|c|c|c|c|}
\hline & $\begin{array}{l}\mathrm{C} \\
\mathrm{H} \\
\mathrm{C} \\
\mathrm{H} \\
\mathrm{H} \\
\mathrm{N} \\
\mathrm{H} \\
\mathrm{H}\end{array}$ & $\begin{array}{r}-0.63417100 \\
-0.48824000 \\
-1.94810500 \\
-2.79181400 \\
-2.16870500 \\
1.82009700 \\
1.97775600 \\
2.62468200 \\
\end{array}$ & $\begin{array}{r}-0.38266300 \\
-1.46698500 \\
0.09284300 \\
-0.59082000 \\
1.15850600 \\
-0.10508400 \\
-1.10269400 \\
0.50193600 \\
\end{array}$ & $\begin{array}{r}0.00000600 \\
-0.00003600 \\
0.00001300 \\
-0.00010200 \\
0.00011700 \\
0.00019700 \\
-0.00036200 \\
-0.00051600 \\
\end{array}$ \\
\hline $\mathrm{H}_{2} \mathrm{~N} \widehat{=}=\mathrm{CH}_{2}$ & $\begin{array}{l}\text { Fr } \\
\text { En } \\
\text { ZF } \\
\text { To } \\
\text { Fr } \\
\\
0 \\
\text { C } \\
\mathrm{H} \\
\mathrm{H} \\
\mathrm{C} \\
\mathrm{H} \\
\mathrm{C} \\
\mathrm{H} \\
\mathrm{H} \\
\mathrm{N} \\
\mathrm{H} \\
\mathrm{H}\end{array}$ & $\begin{array}{l}y:-173.15494 \\
173.121467 \\
\text { ted Energy: }-17 \\
y:-173.225556 \\
117.0114 \\
\\
-0.57106300 \\
-0.38071300 \\
-0.82532700 \\
0.69472600 \\
0.59689300 \\
1.87111200 \\
2.75235100 \\
1.98655400 \\
-1.69893700 \\
-2.62398700 \\
-1.58185800\end{array}$ & $\begin{array}{l}5 \\
73.127612 \\
61 \\
\\
0.53441300 \\
1.38465700 \\
0.96202700 \\
-0.28609300 \\
-1.17511900 \\
0.00796100 \\
-0.61405500 \\
0.88099000 \\
-0.32374100 \\
0.07334000 \\
-0.78333600\end{array}$ & $\begin{array}{r}0.20871800 \\
-0.47352100 \\
1.19029400 \\
0.34657900 \\
0.97136100 \\
-0.24477700 \\
-0.10376000 \\
-0.88609400 \\
-0.19933700 \\
-0.06865000 \\
-1.09739200\end{array}$ \\
\hline $\mathrm{HO} \widehat{=}=\mathrm{CH}_{2}$ & $\begin{array}{l}0 \\
\mathrm{C} \\
\mathrm{H} \\
\mathrm{H} \\
\mathrm{C} \\
\mathrm{H} \\
\mathrm{C} \\
\mathrm{H} \\
\mathrm{H} \\
\mathrm{O} \\
\mathrm{H}\end{array}$ & $\begin{array}{r}-0.57754800 \\
-0.42559900 \\
-0.82973500 \\
0.67021200 \\
0.55087100 \\
1.86707600 \\
2.74334800 \\
2.00634800 \\
-1.65988600 \\
-2.52458800\end{array}$ & $\begin{array}{r}0.49026400 \\
1.41278900 \\
0.76657800 \\
-0.35254900 \\
-1.35060800 \\
0.05725200 \\
-0.58517500 \\
1.04300300 \\
-0.32266400 \\
0.12492400\end{array}$ & $\begin{array}{r}0.26371500 \\
-0.31681200 \\
1.30236600 \\
0.25298500 \\
0.67517800 \\
-0.21124000 \\
-0.16735100 \\
-0.65264900 \\
-0.30995400 \\
-0.19386000\end{array}$ \\
\hline $\mathrm{H}_{2} \mathrm{C} \triangleq \mathrm{CH}_{2}$ & \multicolumn{4}{|c|}{$\begin{array}{l}\text { Free Energy: }-117.204632 \\
\text { Enthalpy: }-117.174746 \\
\text { ZPE Corrected Energy: }-117.179474 \\
\text { Total Energy: }-117.2461049 \\
\text { Frequency: } 427.9163 \\
02 \quad 1.24299000 \quad-0.19839300 \\
C \quad\end{array}$} \\
\hline
\end{tabular}




\begin{tabular}{|l|rrrr|}
\hline & $\mathrm{H}$ & 1.31344100 & -1.28461000 & 0.00011200 \\
& $\mathrm{H}$ & 2.17374800 & 0.36305100 & -0.00054400 \\
$\mathrm{C}$ & -0.00001300 & 0.44749600 & 0.00000000 \\
& $\mathrm{H}$ & -0.00004900 & 1.53923100 & 0.00012500 \\
$\mathrm{C}$ & -1.24303200 & -0.19845400 & -0.00003500 \\
& $\mathrm{H}$ & -2.17366500 & 0.36311400 & 0.00007600 \\
& $\mathrm{H}$ & -1.31315000 & -1.28467900 & -0.00015500 \\
\hline
\end{tabular}

${ }^{1}$ (a) Becke, A. D. Phys Rev A 1988, 38, 3098; (b) Lee, C. T.; Yang, W. T.; Parr, R. G. Phys Rev B 1988, 37, 785 (c) Tantillo, J. Am. Chem. Soc., 2006, 128, 6172 (d) Gutta, P., Tantillo, D. J. Angew. Chem. Int. Ed., 2005, 44, 2719 (e) Singleton, D. A.; Hang, C.; Szymanski, M. J.; Meyer, M. P.; Leach, A. G.; Kuwata, K. T.; Chen, J. S.; Greer, A.; Foote, C. S.; Houk, K. N. J. Am. Chem. Soc., 2003, 125, 1319 (f) Singleton, J. Am. Chem. Soc., 2012, 134, 1914 (g) Davis, R. L.; Leverett, C. A.; Romo, D.; Tantillo, D. J. J. Org. Chem., 2011, 76, 7167. (h) Leverett, C. A.; Purohit, V. C.; Johnson, A. G.; (i) Davis, R. L.; Tantillo, D. J.; Romo, D. J. Am. Chem. Soc., 2012, 134, 13348.

${ }^{2}$ (a) Reed, A. E.; Curtiss, L. A.; Weinhold, F. Chem. Rev., 1988, 88, 899. (b) Weinhold F. in Schleyer P.v.R. Encyclopedia of Computational Chemistry: Wiley: New-York, 1998, 3, 1792. (c) Reed, A. E.; Weinhold, F. Isr. J. Chem., 1991, 31, 277. (d) Weinhold, F.; Landis, C. R.; Glendening, E. D. International Reviews in Physical Chemistry 2016, 35, 1. (e) Reed, A. E.; Weinhold, F. J. Chem. Phys., 1985, 83, 1736. 